\title{
A Game Theoretic Approach for Security and Quality of Service (QoS) Co-Design in Cooperative Wireless Communication Networks
}

by

Du Zheng

A dissertation submitted to the

Faculty of Graduate Studies and Research

in partial fulfillment of the requirements for the degree of

Master of Applied Science in Electrical and Computer Engineering

Ottawa-Carleton Institute for Electrical and Computer Engineering (OCIECE)

Department of Systems and Computer Engineering

Carleton University

Ottawa, Ontario, Canada, K1S 5B6

December, 2011

(C)Copyright 2011, Du Zheng 
Library and Archives

Canada

Published Heritage

Branch

395 Wellington Street

Ottawa ON K1A ON4

Canada
Bibliothèque et

Archives Canada

Direction du

Patrimoine de l'édition

395 , rue Wellington

Ottawa ON K1A ON4

Canada
Your file Votre référence

ISBN: 978-0-494-87829-3

Our file Notre référence

ISBN: $978-0-494-87829-3$

\section{NOTICE:}

The author has granted a nonexclusive license allowing Library and Archives Canada to reproduce, publish, archive, preserve, conserve, communicate to the public by telecommunication or on the Internet, loan, distrbute and sell theses worldwide, for commercial or noncommercial purposes, in microform, paper, electronic and/or any other formats.

The author retains copyright ownership and moral rights in this thesis. Neither the thesis nor substantial extracts from it may be printed or otherwise reproduced without the author's permission.
AVIS:

L'auteur a accordé une licence non exclusive permettant à la Bibliothèque et Archives Canada de reproduire, publier, archiver, sauvegarder, conserver, transmettre au public par télécommunication ou par l'Internet, prêter, distribuer et vendre des thèses partout dans le monde, à des fins commerciales ou autres, sur support microforme, papier, électronique et/ou autres formats.

L'auteur conserve la propriété du droit d'auteur et des droits moraux qui protege cette thèse. $\mathrm{Ni}$ la thèse ni des extraits substantiels de celle-ci ne doivent être imprimés ou autrement reproduits sans son autorisation.
In compliance with the Canadian Privacy Act some supporting forms may have been removed from this thesis.

While these forms may be included in the document page count, their removal does not represent any loss of content from the thesis.
Conformément à la loi canadienne sur la protection de la vie privée, quelques formulaires secondaires ont été enlevés de cette thèse.

Bien que ces formulaires aient inclus dans la pagination, il n'y aura aucun contenu manquant. 


\section{Abstract}

Cooperative communication is proposed to form a virtual MIMO system through strategic relay selection to improve communication quality in wireless communication networks. Therefore, relay selection exerts a significant impact on improving communication quality in wireless communication networks. Due to its unorganized and decentralized infrastructure, cooperative wireless communication networks are vulnerable to the attacks initiated on relays. Though encryption and authentication protocols can ensure reliable data transmission when the selected relay is compromised by the attacker in vulnerable cooperative wireless communication networks, limited system resource is wasted by applying encryption and authentication protocols when the selected relay is not selected as attacking target and compromised by the attacker. In this thesis, a game theoretic approach is proposed to quantitatively analyze the attacking strategies of the attacker so as to make decision on relay selection and the extent of applying encryption and authentication protocols to reach the trade-off between security and Quality of Service (QoS) in cooperative wireless communication networks. Simulation results show the effectiveness of the proposed game theoretic approach for security and QoS co-design in cooperative wireless communication networks. 
To my parents, sister and wife 


\section{Acknowledgments}

The author wishes to express his sincere appreciation to his supervisor, Dr. F. Richard Yu and co-supervisor, Dr. Helen Tang. He is grateful to their support, encouragement and invaluable advice.

Special thanks are due to his respected friend Dr. Yuanbo Guo for his valuable advice. The author expresses his thanks to his colleagues Mrs. Shuhuan Wen, Mrs. Shengrong Bu, Mr. Renchao Xie, Mr. Yegui Cai and the other graduate students in OCIECE for their assistance and happy time spent together.

Thanks are also due to the faculty and staff in Department of Systems and Computer Engineering for providing support to his graduate study and thesis experiment. 


\section{Table of Contents}

Abstract $\quad$ iii

Acknowledgments $\quad$ v

Table of Contents vi vi vis

$\begin{array}{ll}\text { List of Tables } & \text { ix }\end{array}$

List of Figures $\quad \mathbf{x}$

List of Abbreviations $\quad$ xii

List of Symbols $\quad$ xiv

1 Introduction 1

1.1 Research Overview ...................... 1

1.2 Research Motivation . . . . . . . . . . . . . . . 2

1.3 Research Objectives . . . . . . . . . . . . . . . . 2

1.4 Thesis Contributions . . . . . . . . . . . . . . 3

1.4.1 Submitted Paper . . . . . . . . . . . . . . . 5

1.5 Thesis Organization . . . . . . . . . . . . . 5

$\begin{array}{lll}2 & \text { Background and Related Work } & 7\end{array}$ 
2.1 Cooperative Communication . . . . . . . . . . . . 7

2.1.1 Cooperative Signaling Methods . . . . . . . . . . 9

2.1.2 Opportunistic Relaying . . . . . . . . . . . . . 10

2.2 Security in Cooperative Wireless Communication Networks . . . . . 12

2.3 ALPHA: An Adaptive and Lightweight Protocol for Hop-by-hop Au-

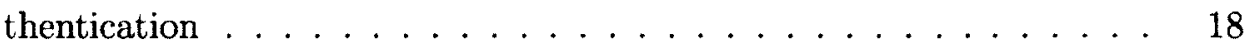

2.3 .1 Basic ALPHA . . . . . . . . . . . . . . 19

2.3.2 ALPHA-M: ALPHA-with Pre-signed Merkle Tree . . . . . . 20

2.4 Game Theory . . . . . . . . . . . . . . . . . . . 22

2.4.1 Static Game and Dynamic Bayesian Game . . . . . . . . . 24

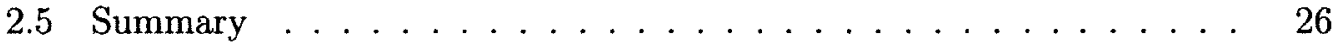

3 Proposed Game Theoretic Approach $\quad 27$

3.1 Model Description . . . . . . . . . . . . . . 27

3.2 Game Played for Security and QoS Co-Design . . . . . . . . . . . 28

3.2.1 Static Game Theoretic Approach . . . . . . . . . . . . 29

3.2.2 Dynamic Bayesian Game Theoretic Approach . . . . . . . 31

3.2.3 Bayesian Updating Rule on Beliefs in the Maliciousness of Relays 32

3.3 Find Nash Equilibrium of the Proposed Game Theoretic Approach . . 34

3.3.1 Find Nash Equilibrium of the Proposed Static Game Theoretic Approach ....................... 36

3.3.2 Find Nash Equilibrium of the Proposed Dynamic Game Theoretic Approach . . . . . . . . . . . . . . 38

3.4 System Performance Analysis . . . . . . . . . . . . . . . 41

3.4.1 Outage Probability and Capacity . . . . . . . . . . . 42

3.4 .2 Bit Error Rate . . . . . . . . . . . . . . . . . 44

3.4 .3 System Throughput . . . . . . . . . . . . . . 45 
3.4.4 Optimizing Number of Message ............ 51

4 Simulation Results and Discussion 53

4.1 Simulation Scenarios . . . . . . . . . . . . . 53

4.2 Optimal Number of Messages _. . . . . . . . . . . 56

4.3 Effect of Processing Time on the Optimal Number of Messages . . . . 56

4.4 Effect of Authentication Probability on Throughput and Compromising Probability ..................... 57

4.5 Dynamic Beliefs in the Maliciousness of Relays . . . . . . . . . . 59

4.6 Impact of Dynamic Belief Update in the Maliciousness of Relays on Throughput and Compromising Probability . . . . . . . . 62

4.7 Summary . . . . . . . . . . . . . . . . 66

5 Conclusions and Future Work $\quad 72$

5.1 Conclusions . . . . . . . . . . . . . . . . 72

5.2 Future Work . . . . . . . . . . . . . . 73

$\begin{array}{lr}\text { List of References } & 75\end{array}$

$\begin{array}{lr}\text { Appendix A Simulation Programs } & \mathbf{8 0}\end{array}$ 


\section{List of Tables}

3 Utility matrix of attacker and source on relay $R_{i} \ldots \ldots 30$

4 Utility matrix of attacker and source on relay $R_{i}$ with probability $\mu_{i}^{t_{k}}$ being malicious at stage $t_{k} \ldots \ldots \ldots \ldots \ldots \ldots \ldots$

5 Utility matrix of attacker and source on relay $R_{i}$ with probability $1-\mu_{i}^{t_{k}}$ being cooperative at stage $t_{k} \ldots \ldots \ldots \ldots \ldots$

6 Utility matrix of attacker and source on relay $R_{i}$ at stage $t_{k}$ after Harsanyi transformation . . . . . . . . . . . . . . 38

7 Time parameters in $T_{1} \ldots \ldots \ldots \ldots$

8 Time parameters in $T_{2} \ldots \ldots \ldots \ldots \ldots$

9 Nash equilibrium and players' utility in the military network . . . . 55

10 Nash equilibrium and players' utility in the commercial network ... 55 


\section{List of Figures}

1 Cooperative communication ............... 8

2 Basic ALPHA .......................... 20

3 Merkle tree with 8 leaves . . . . . . . . . . . . . . 21

4 Acknowledgments Merkle tree . . . . . . . . . . . . . 22

$5 \quad$ ALPHA-M with Pre-(n)acks . . . . . . . . . . . . . 23

6 A cooperative wireless communication network . . . . . . . . 28

7 Message sequence charts in direct communication and source-relaydestination communication . . . . . . . . . . . . . 52

8 Simulation topology . . . . . . . . . . . . . . . 54

9 The effect of the number of messages on system throughput . . . . 57

10 Change in optimal $n$ for different processing time of terminals in military network, $p_{a}=0.75 \ldots \ldots \ldots \ldots \ldots$

11 Change in optimal $n$ for different processing time of terminals in commercial network, $p_{a}=0.15 \ldots \ldots \ldots . \ldots . \ldots 59$

12 Effect of authentication probability on throughput of military network, $\mathrm{SNR}=30 \mathrm{~dB} \ldots \ldots \ldots \ldots \ldots$

13 Effect of authentication probability on throughput of commercial network, $\mathrm{SNR}=30 \mathrm{~dB} \ldots \ldots \ldots \ldots$

14 Effect of authentication probability on compromising probability of military and commercial networks . . . . . . . . . . . 62 
15 Dynamic attacking target and selected relay . . . . . . . . 63

16 Dynamic belief in the maliciousness of Relay $1 \ldots 63$

17 Dynamic belief in the maliciousness of Relay 2 . . . . . . . . . 64

18 Dynamic belief in the maliciousness of Relay $3 \ldots 64$

19 Dynamic belief in the maliciousness of Relay $4 \ldots \ldots 5$

20 Dynamic total utility of the source in military network . . . . . . 66

21 Dynamic total utility of the source in commercial network . . . . . 67

22 Comparison of dynamic total utility of the source in military and commercial networks . . . . . . . . . . . . . 68

23 Impact of dynamic belief update in the maliciousness of relays on compromising probability of military network . . . . . . . . . 68

24 Impact of dynamic belief update in the maliciousness of relays on compromising probability of commercial network . . . . . . . . 69

25 Dynamic compromising probability comparison between military and commercial network . . . . . . . . . . . . . . . 69

26 Impact of dynamic belief update in the maliciousness of relays on throughput of military network, SNR $=30 \mathrm{~dB} \ldots \ldots 70$

27 Impact of dynamic belief update in the maliciousness of relays on throughput of commercial network, SNR $=30 \mathrm{~dB} \ldots 70$

28 Dynamic throughput comparison between military and commercial network, SNR $=30 \mathrm{~dB} \ldots \ldots \ldots \ldots \ldots \ldots$ 


\section{List of Abbreviations}

ack Positive Acknowledgment

nack Negative Acknowledgment

AF Amplify-and-Forward

ALPHA Adaptive and Lightweight Protocol for Hop-by-hop Authentication

ALPHA-M ALPHA with Pre-signed Merkle Tree

AMT Acknowledgment Merkle Tree

ARQ Automatic Repeat reQuest

BER Bit Error Rate

BPSK Binary Phase Shift Keying

CC Coded Cooperation

DC Direct Communication

DF Decode-and-Forward or Detect-and-Forward

ECC Elliptic Curve Cryptosystem

FEC Forward Error Correction

FSDF Fixed Selective Decode-and-Forward

GBN Go-Back-N

HEAP Hop-by-Hop Efficient Authentication Protocol

HMAC Hash Message Authentication Code

IBE Identity Based Encryption

ITU International Telecommunications Union

LHAP Lightweight Hop-by-Hop Authentication Protocol

MAC Message Authentication Code 
MANET Mobile Ad hoc Network

MRC Maximal Ratio Combining

MIMO Multiple-Input Multiple-Output

MT Merkle Tree

QoS Quality of Service

SC Smart Cooperation/Smart Cooperative

SHA Secure Hash Algorithm

SNR Signal-to-Noise Ratio

SR Selective Repeat

SSDF Smart Selective Decode-and-Forward

SW Stop and Wait

TESLA Timed Efficient Stream Loss-tolerant Authentication

TTP Trusted Third Party

WLAN Wireless Local Area Network

WSN Wireless Sensor Network 


\section{List of Symbols}

$\begin{array}{ll}a & \text { Indicator of Acknowledgment Hash Chain } \\ c & \text { Speed of Light } \\ d_{R_{i} D} & \text { Distance between Relay } R_{i} \text { and Destination } \\ d_{S D} & \text { Distance between Source and Destination } \\ d_{S R_{i}} & \text { Distance between Source and Relay } R_{i} \\ f_{i}^{t_{k-1}} & \text { Priori Probability Density Function on Relay } R_{i} \text { at Stage } t_{k-1} \\ h_{i} & \text { Hash Chain Anchor } \\ h_{i}^{S s} & \text { Anchor of Signature Hash Chain of Source } \\ h_{i}^{S a} & \text { Anchor of Acknowledgment Hash Chain of Source } \\ h_{i}^{D s} & \text { Anchor of Signature Hash Chain of Destination } \\ h_{i}^{S a} & \text { Anchor of Acknowledgment Hash Chain of Destination } \\ \left|h_{S D}\right| & \text { Channel between Source and Destination } \\ \left|h_{S R_{i}}\right| & \text { Channel between Source and Relay } R_{i} \\ \left|h_{R_{i} D}\right| & \text { Channel between Relay } R_{i} \text { and Destination } \\ i & \text { Hash Chain Length } \\ m & \text { Message } \\ m_{j} & \text { Message Blocks } \\ n & \text { Number of Messages or Data Blocks } \\ n^{*} & \text { Optimal Number of Messages } \\ p_{a} & \text { Probability of Message Authentication } \\ p_{i} & \text { Attacker's Attacking Probability on Relay } R_{i} \\ & \text { System Security Requirement } \\ & \end{array}$

xiv 
$q_{i}$

$r$

$r^{\prime}$

$s$

$t_{a c k 1}$

$t_{a c k 2}$

$t_{f 1}$

$t_{f 2}$

$t_{\text {proc1 }}$

$t_{\text {proc } 2}$

$t_{\text {prop } 1}$

$t_{\text {prop2 }}$

$u_{\text {ack } 1}$

$u_{a c k 2}$

$u_{f 1}$

$u_{f 2}$

$x_{i}$

$A_{1}$

$A_{2}$

$\left\{B_{c}\right\}$

$C_{c}^{S C, i}$

$C_{a}$

$C_{f}$

$C_{m}$

D

H

$I_{i}$

$I_{S C}$

$I_{S R_{i}}$

Source's Selection Probability on Relay $R_{i}$

Data Transmission Rate

Random Number

Indicator of Signature Hash Chain

Packet Transmission Time for $A_{1}$

Packet Transmission Time for $A_{2}$

Packet Transmission Time for $S_{1}$

Packet Transmission Time for $S_{2}$

Processing Time in $T_{1}$

Processing Time in $T_{2}$

Propagation Time for $S_{1}$

Propagation Time for $S_{2}$

Number of Bits in $A_{1}$

Number of Bits in $A_{2}$

Number of Bits in $S_{1}$

Number of Bits in $S_{2}$

Index of Packet

Acknowledgment Pre-signature Initial Packet Sent by Destination

Acknowledgment Packet Sent by Destination

Set of Sibling Nodes on the Path from Message to Root in Merkle Tree

Outage Capacity for Relay $R_{i}$ in SC Relaying System

Cost for Attacker by Attacking Targeted Relay

Cost for Source by Fa'se Attack Monitoring

Cost for Source by Monitoring Attack on Selected Relay

Destination

One-way Hash Function

Mutual Information between Source and Destination through Relay $R_{i}$

Mutual Information in Smart Cooperative Relaying System

Mutual Information between Source and Relay $R_{i}$ 


\begin{tabular}{|c|c|}
\hline$I_{M R C}$ & Mutual Information between Source-Destination and Destination-Relay \\
\hline$M_{i}^{t_{k}}$ & No. of Packets Sent by Source through Relay $R_{i}$ at Stage $t_{k}$ \\
\hline$N_{i}^{t_{k}}$ & No. of Packets Successfully Forwarded through Relay $R_{i}$ at Stage $t_{k}$ \\
\hline$P$ & Attacker's Attacking Probability Distribution \\
\hline$P_{c}$ & Packet Error Rate \\
\hline$P_{e}^{d i v, i}$ & Probability of Error in Combined Transmission \\
\hline$P_{e}^{S C, i}$ & End-to-end Bit Error Rate Through Relay $R_{i}$ in SC Relaying System \\
\hline$P_{e}^{S D}$ & Probability of Error in Direct Transmission \\
\hline$P_{\text {out }}^{I}$ & Outage Probability \\
\hline$P_{\text {out }}^{S C, i}$ & Outage Probability through Relay $R_{i}$ in SC Relaying System \\
\hline$P_{\text {out }}^{S R_{i}}$ & Outage Probability from Source to Relay $R_{i}$ \\
\hline$Q$ & Source's Relay Selection Probability Distribution \\
\hline$R_{i}$ & Relay $i$ \\
\hline$S$ & Source \\
\hline$S_{h}$ & Hash Output \\
\hline$S_{1}$ & Initial Packet Containing Pre-signature Sent by Source \\
\hline$S_{2}$ & Message Packet Sent by Source \\
\hline$S_{\text {payload }}$ & Payload for Packets with Authentication \\
\hline$S_{\text {paylaod }}^{\prime}$ & Payload for Packets without Authentication \\
\hline$S_{\text {packet }}$ & Size of Packet \\
\hline$S R D$ & Source-Relay-Destination \\
\hline$T_{1}$ & Time for Initial Pre-signature Process between Source and Destination \\
\hline$T_{2}$ & Time for Actual Message Transmission and Delivery \\
\hline$T_{i}^{t_{k}}$ & Trust Value on Relay $R_{i}$ at Stage $t_{k}$ \\
\hline$U_{A}(P, Q)$ & Total Utility of Attacker in Proposed Game Theoretic Approach \\
\hline$U_{S}(P, Q)$ & Total Utility of Source in Proposed Game Theoretic Approach \\
\hline$W_{s}$ & Window Size \\
\hline$\alpha$ & Path Loss Exponent \\
\hline$\alpha_{I}$ & Weight of Information Asset in Asset Combination $\alpha_{I} I_{i}+\alpha_{S} S_{i}$ \\
\hline
\end{tabular}




$\begin{array}{ll}\alpha_{S} & \text { Weight of Security Asset in Asset Combination } \alpha_{I} I_{i}+\alpha_{S} S_{i} \\ \alpha_{I} I_{i}+\alpha_{S} S_{i} & \text { Asset Combination of Information Asset and Security Asset } \\ \bar{\gamma} & \text { Average Received Signal-to-Noise Ratio } \\ \overline{\gamma_{R_{k} D}} & \text { Average Received SNR between Relay and Destination } \\ \overline{\gamma_{S D}} & \text { Average Received SNR between Source and Destination } \\ \overline{\gamma_{S R_{k}}} & \text { Average Received SNR between Source and Relay } \\ \mu_{i}^{t_{k}} & \text { Priori Maliciousness Belief Probability on Relay } R_{i} \text { at Stage } t_{k} \\ \varepsilon & \text { Fixed Outage Probability } \\ \theta & \text { Hash Chain Random Seed Variable } \\ \mathcal{R} & \text { Set of Relays } \\ \left|\mathcal{R}_{S}\right| & \text { Sensible Target Set } \\ \left|\mathcal{R}_{Q}\right| & \text { Quasi-sensible Target Set } \\ \left|\mathcal{R}_{N}\right| & \text { Non-sensible Target Set }\end{array}$

xvii 


\section{Chapter 1}

\section{Introduction}

\subsection{Research Overview}

Cooperative communication has been assumed to improve communication quality in wireless communication networks through strategic relay selection to form a virtual multiple-input and multiple-output (MIMO) system. However, cooperative wireless communication networks are vulnerable to the attacks on relays. Though encryption and authentication protocols can secure cooperative communication when selected relays by the source are selected as attacking target by the attacker as well, it results in the waste of system resource when selected relays are cooperative. In this thesis, we propose a game theoretic approach for security and QoS co-design in cooperative wireless communication networks. The proposed game theoretic approach for security and QoS co-design in cooperative wireless communication networks provides a quantitative decision framework on relay selection, which highlights the trade-off between system security and system throughput. Simulation results show the effectiveness of the proposed approach for the security and QoS co-design. 


\subsection{Research Motivation}

The motivation for initiating this research by applying game theory for security and QoS co-design in cooperative wireless communication networks originates from following problems met in cooperative wireless communication networks.

As mentioned earlier, cooperative wireless communication networks are vulnerable to the attack on relays. Though encryption and stringent authentication protocols are effective to combat the attack initiated by the attacker on relays in cooperative wireless communication networks, system security could be provided at the cost of introducing tremendous overheads into the system due to the execution of encryption and stringent authentication protocols. When the selected relays are cooperative and perform as what they are expected to, applying encryption and stringent authentication protocols is kind of wasting limited system resource. Thus, it is necessary to provide a quantitative approach to analyze the attacking action of the attacker so as to make right relay selection decision and extent of applying encryption and authentication protocols.

To solve above mentioned problems met in cooperative wireless communication networks, an approach based on game theory is proposed to quantitatively model the interactions between the attacker's attacking target selection and the source's relay selection and obtain the extent of applying encryption and authentication protocols in this thesis.

\subsection{Research Objectives}

The main objective of this research is to design a game theoretic approach for security and QoS co-design in cooperative wireless communication networks which considers the trade-off between system security and system throughput. More concisely, our 
objectives are shown as below:

- To evaluate existing approaches' coverage on security and QoS in cooperative wireless communication networks.

- To propose a quantitative decision making approach which is based on game theory and takes both security and QoS into consideration.

- To compare the simulation results obtained by applying the proposed approach with the simulation results achieved by applying existing approaches to verify the effectiveness of the proposed approach for security and QoS co-design.

\subsection{Thesis Contributions}

Based on the objectives mentioned above, we propose a game theoretic approach for security and QoS co-design in cooperative wireless communication networks. Following are the thesis contributions:

- A game theoretic approach for security and QoS co-design in cooperative wireless networks is proposed for two-hop cooperative wireless networks with three types of nodes, i.e., source, destination and relays, from which the one that brings source the maximum utility is selected to forward message sent from source to destination.

- A static game theoretic approach, with the assumption that all the relays are malicious, is proposed to model the scenarios in cooperative wireless communication networks in which all relays are the attacking victims of the attacker.

- A dynamic Bayesian game theoretic approach is proposed to overcome the weakness of the proposed static game theoretic approach, which neglects the existence of cooperative relays; and provide the source a dynamic decision making 
approach by updating its beliefs in the maliciousness of selected relays based on its original beliefs and record of the attacker's attacking histories on selected relays.

- The proposed game theoretic approach for security and QoS co-design, which tries to balance system security and system throughput in cooperative wireless communication networks, overcomes the weakness of existing approaches that either overemphasize on security at the cost of degraded system performance or emphasize on system performance improvement but fail to take security issues into consideration.

- According to the weight of each relay's asset over the overall assets of all relays, attacking targets are categorized into three categories, sensible set, quasisensible set and non-sensible set. Simulation results show that not only the attacker selects its attacking target from the sensible set and quasi-sensible set, but also the source selects its relay from the sensible and quasi-sensible set.

- According to attacker's attacking probability on selected relay which is obtained by finding the Nash equilibrium of the proposed game theoretic approach and meeting system security requirement, a minimal authentication probability on selected relay is derived. A light-weight authentication protocol, ALPHA, which is based on hash chains and Merkle trees, is applied to assure not only hop-byhop but also end-to-end identity authentication and integrity protection.

- Throughput equations derived from the proposed game theoretic approach for security and QoS co-design in cooperative wireless communication networks incorporate Selective Repeated ARQ and Go-Back-N ARQ error control schemes. 


\subsubsection{Submitted Paper}

The following paper has been submitted:

- D. Zheng, H. Tang and F. R. Yu, "A Game Theoretic Approach for Security and Quality of Service (QoS) Co-Design in Cooperative Wireless Communication Networks", submitted to IEEE Milcom'12, Orlando, FL.

\subsection{Thesis Organization}

The rest of the thesis is organized as follows:

- Chapter 2 describes the background of the research presented in this thesis, in which concepts of cooperative wireless communication, cooperative signaling methods, opportunistic relaying, security issues met in cooperative wireless communication networks and existing encryption and authentication protocols to assure security in cooperative wireless communication networks are included.

- Chapter 3 presents a game theoretic approach for security and QoS co-design in cooperative wireless communication networks. The proposed game theoretic approach includes a static game theoretic approach to cope with the scenarios, in which all relays are malicious; and a dynamic Bayesian game theoretic approach to cope with the scenarios, in which not all relays are malicious and the attacker dynamically selects its attacking targets. Chapter 3 also describes the utility functions used in the proposed game theoretic approach, algorithm for finding Nash equilibrium of the proposed game theoretic approach, criteria for relay selection and system performance analysis.

- Chapter 4 presents the simulation results obtained by applying the proposed 
game theoretic approach for security and QoS co-design in cooperative wireless communication networks. Discussions on comparing obtained simulation results by applying the proposed game theoretic approach with that obtained by applying existing approach [1] are also presented.

- Chapter 5 wraps up the research conclusions and future research orientation.

The simulation programs are presented in Appendix A. 


\section{Chapter 2}

\section{Background and Related Work}

\subsection{Cooperative Communication}

The advantages of MIMO systems have been widely acknowledged; to the extent that certain transmit diversity methods have been incorporated into wireless standards. Though transmit diversity is clearly advantageous on a cellular base stations, it may not be pragmatic for other applications. Obviously, under the limitation of size, cost, or hardware implementation, a wireless device may not be able to support multiple transmit antennas, such as most handsets, because of the limitation of set size or the nodes in a wireless sensor networks, due to the limitation of size and power. Cooperative communication allows single-antenna mobiles to reap some of the benefits of MIMO systems. The fundamental idea behind cooperative communication is that single-antenna mobiles in a multi-user scenario can share their antennas in a manner that creates a virtual MIMO system. It is well-known that the mobile wireless channel suffers from fading; in another word, the signal attenuation can vary significantly over the course of a given transmission. Transmitting independent copies of the signal generates diversity and can effectively combat the deleterious effects of fading. Particularly, spatial diversity is generated by transmitting signals from 


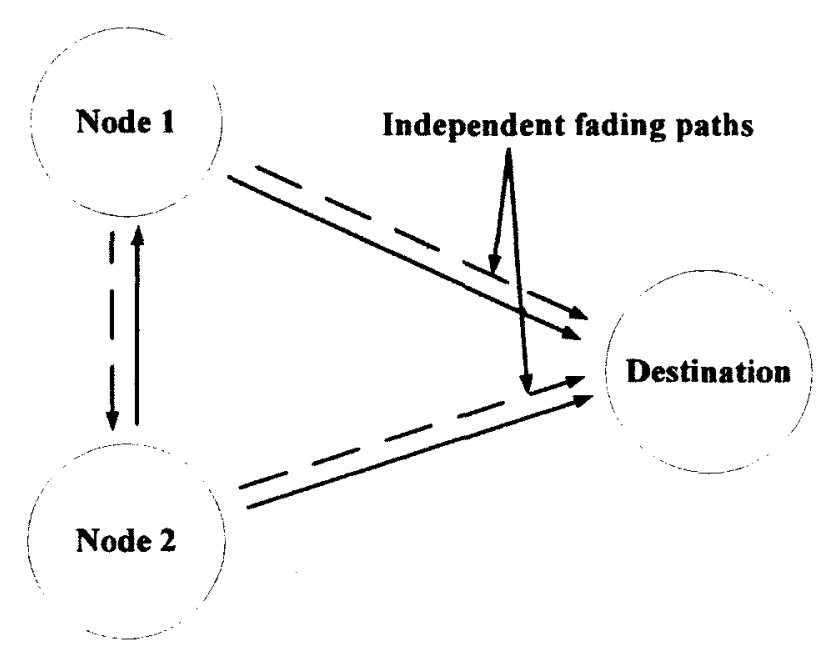

Figure 1: Cooperative communication

different locations, thus allowing independently faded versions of the signal arrive at the receiver. Cooperative communication generates spatial diversity in a new and interesting way. As illustrated in Figure 1, in which node represents mobile device with one antenna, two nodes are communicating with the same destination. Each node has one antenna and cannot individually realize spatial diversity. However, it is possible for one node to receive the information sent from the other, in which case it could forward received information along with its own information to the destination. Since the fading path from two nodes is statistically independent, spatial diversity is achieved [2].

In this study, we consider the cellular or Ad hoc networks, in which users may increase their effective quality of services through cooperation. Each wireless user is assumed to transmit data as well as function as cooperative relay to forward received data from its partners. 


\subsubsection{Cooperative Signaling Methods}

Several of main cooperative signaling methods used in cooperative wireless communication networks are presented below.

\section{Amplify and Forward (AF)}

In this method, each node receives a noisy version of the signal transmitted by its partner. The node then amplifies and retransmits this received noisy version of signal to the destination. The destination combines the signal sent by the node and the node's partner, and makes a final decision on the transmitted information. Although noise is amplified by cooperation, the base station receives two independently faded versions of signal and can make better decisions on the detection of information compared with using only the version received from one node [3].

\section{Detect and Forward (DF)}

In this method, a node attempts to detect the partner's bits and then retransmits the detected bits if the received signal could be decoded successfully. Compared with the amplify and forward method, detect and forward method adds some implementation complexity, but exhibits better performance especially in low signal-to-noise ratios compared with amplify and forward method. Detect and forward is also named as decode and forward, which not only deals with single relay forwarding the received signal to the destination, but also handles multiple relays forwarding the received signal simultaneously to the destination [2].

\section{Coded Cooperation (CC)}

Coded cooperation integrates cooperation into channel coding. Coded cooperation works by sending different portions of each node's code word via independent fading 
paths. The basic idea is that each node tries to transmit incremental redundancy to its partner. Whenever that is not possible, the nodes automatically revert to a noncooperative mode. The key to the efficiency of coded cooperation is that all this is managed automatically through code design, with no feedback between the nodes [4].

\subsubsection{Opportunistic Relaying}

The proposed game theoretic approach for security and QoS co-design in cooperative wireless communication networks in this thesis adopts a proactive opportunistic relaying process with DF signaling. As the name implies, opportunistic relaying selects the best relay according to different relay selection criteria among all candidate relays to forward the signal between the source and the destination [5]. An opportunistic relaying process consists of two time slots. In the first time slot, the source broadcasts the signal which could be heard by all relay nodes in its radio coverage and the destination; in the second time slot, if the signal received by the selected relay node could be decoded successfully, it would be forwarded to the destination; then the destination combines the received signal from the source and selected relay to recover the information sent from the source.

For opportunistic relaying, there are two typical relay selection schemes, proactive selection and reactive selection. In proactive relay selection, the source selects the best relay before transmitting the data from the source to the destination [6]. There is no requirement on all intermediate relays to listen to the source's broadcasting except for the selected relay, thus power or energy spent by unselected relays on listening to the channel and receiving the message sent by the source is saved. In proactive relay selection, only the selected relay is required to keep its ears open to listen to the channel, thus only a small amount of energy is needed by adopting proactive selection. In reactive relay selection, all intermediate relay nodes are involved in listening to the 
signal transmitted by the source in the first time slot. Only after all intermediate relay nodes receive the signal sent by the source, the best relay is selected out to forward the received signal sent by the source to the destination [5]. Since all the intermediate relays need listen to the signal broadcasted by the source, energy utilized in reactive relay selection is obviously more than that utilized in proactive relay selection. There are three proactive diversity schemes: Fixed Selective Decode-and-Forward (FSDF) with direct link combining, Fixed Selective Decode-and-Forward (FSDF) without direct link combining and Smart Selective Decode-and-Forward (SSDF) [7].

- FSDF with direct link combining: This scheme optimally selects one relay to decode. If the decoding fails, the transmission is considered as unsuccessful. If selected relay successfully decodes the received signal, it forwards the information to the destination. The destination combines the signals from both the source and the selected relay using Maximum Ratio Combining (MRC).

- FSDF without direct link combining: This scheme is similar to the previous scheme, except for that there is no direct link. If the selected relay could successfully decode the received signal, it forwards the information to the destination node; otherwise, the transmission is considered as failed. The destination only could make use of the signal sent from the selected relay to recover the information sent by the source.

- SSDF: This scheme is similar to the FSDF scheme with direct link combining, but selected relay is not used when direct communication gives larger instantaneous mutual information which saves the transmission time from the source to the destination through the selected relay.

For systems employing such diversity schemes in slow-fading channels, outage 
probability and outage capacity are vital performance measures. In [7], exact expressions for outage probability and outage capacities of three above mentioned proactive cooperative diversity schemes are given. The derived expressions are valid for arbitrary network topology and operating SNR, and serve as efficient tools for cooperative wireless communication networks design. In the next chapter, detailed description of the derived expressions will be presented.

\subsection{Security in Cooperative Wireless Communica- tion Networks}

It is evident that cooperative communication brings significant benefit in improving the communication quality of wireless communication networks. Nodes in cooperative wireless communication networks could be the source, the destination, or the relay. Cooperative wireless communication was originally designed with the assumption that all the nodes involved always help each other and cooperate in a socially efficient manner. However, assumption on complete cooperation is broken by the facts that there exist relays that are attacked by the network attackers and misbehave for selfish or malicious intentions. Security issues met in cooperative wireless communication networks are similar to the security issues met in mobile Ad-hoc networks (MANETs) and wireless sensor networks (WSNs) where cooperation among nodes is also needed.

The following properties of cooperative communication cause vulnerability to the attack which results in poor and disrupted cooperation of nodes in cooperative wireless communication networks [8]:

- Possible Free-riders: one of the main assumptions enabling the cooperative wireless networks to work properly is that nodes are willing to share their resources with other entities in the network. As such, they have to cooperate with other 
nodes when they are expected and selected to do so. However, there is no assurance of the cooperation of the entities. In fact, a node may deliberately refuse to cooperate and relay a partner's information. In this case, an incentive may be considered for well-behaved nodes. To discourage the nodes which do not cooperate, well-behaved nodes can refuse to cooperate with a node without the assurance stating that the node can be trusted in terms of reciprocation.

- Absence of Centralized Control: nodes can move from area to area, from network to network and make relationships which may be short term. It is not feasible to get the complete knowledge of these relationships in advance. Therefore, by authentication, only those legitimate nodes can be allowed entering the network. Also, there should be a mechanism for dynamic monitoring of the nodes' behavior.

- Frequent Topology Changes: possible changes in network topologies make it difficult to prevent misbehavior of relays. All nodes involved in cooperative wireless communication networks must be reliable to provide successful transmission between source and destination. There is no fixed topology of the network and the topology could change frequently.

- Nature of Wireless Medium: the nature of wireless medium causes vulnerability in the way that it is open to any access. In fact, there is no limitation in physical access and therefore, all nodes should be authenticated and monitored in various aspects.

- Resource Constraints: limited power resources and limited orthogonal relaying channels are two examples of the resource constraints in cooperative wireless communication networks. Wasting power and other resources is considered misbehavior in this type of network. In such environment it will not be easy to 
implement high complexity securing solutions.

- Scalability: any policy or protocol should be compatible to the inherent dynamic nature of the incoming relays and should be extensible in the network.

Thus, it is acknowledged that security is one of the main concerns for cooperative communication. The attributes of security concerns related to the security goals mentioned above are defined by the International Telecommunications Union Telecommunication Standardization Sector (ITU-T) as follows [9] [10]:

- Data Confidentiality: to make sure that the data cannot be accessed by unauthorized users or nodes who are not the designated recipients, which could be generally achieved through cryptography.

- Data Integrity: to assure that the data has not been modified during the transmission process, which could be generally achieved through the use of hash functions.

- Authentication: to prevent any impersonation of a user or node in a network and to assist in correcting the identification of an entity.

- Non-repudiation: to make sure entities admit the ownership of their messages which be performed by verifying that the messages were sent with the node's credentials, which could be usually achieved through public key cryptography.

- Access Control: to provide protection against unauthorized access to data and to ensure that legitimate entities access the network.

Above discussed security issues show the importance of data integrity checking and the need to have recognized reliable relationship amongst the different nodes in cooperative wireless communication networks. Authentication is a process that 
involves in a communication process between an authenticator and supplicant to identify the identity of supplicant. Sometimes a Trusted Third Party (TTP) might be involved in an authentication process. Therefore, authentication is important, with the consequent need to know exactly who we are talking to and make sure that the message received from a node is exact the message that had been sent by that node. Authentication, therefore, supports privacy, confidentiality, and access control by verifying and validating the received message. All nodes in the cooperative wireless communication networks should be able to carry out the authentication and act as authenticator and supplicant from time to time.

[11] makes a survey that focuses on node-to-node authentication for wireless communication networks and classifies authentication taxonomy based on the type of credentials. Credentials can be classified into two classes: identity-based and contextbased. Identity-based credentials can be further classified into encryption-based and non-encryption-based.

For encryption-based credentials, the supplicant encrypts a piece of information by using the key processed to verify its identity. In order to verify the supplicant's identity, the authenticator must either process the same key if symmetric key cryptography is applied, or the corresponding public key of the private key used by the supplicant to encrypt the information that is to be verified by authenticator. Symmetric key based authentication is commonly used in wireless sensor networks because of its light computational complexity. In symmetric encryption algorithm, both supplicant and authenticator use the same unique secret key for both encryption and decryption. Both parties share the secret that is unique between these two nodes, and is not known to any other node. Each node stores the unique keys that define the encrypted relationships with other nodes. Symmetric key based authentication is not scalable and is difficult to maintain for a larger network since it is hard to 
refresh the keys. The key must remain secret at both ends and must be changed frequently leading to the need to manage a huge number of key pairs for larger networks. Distributing the shared keys becomes a problem in networks that do not have any centralized control, such as in cooperative or Ad hoc networks. Commonly used symmetric algorithms include Advanced Encryption Standard (AES), Data Encryption Standard (DES). On the other hand, asymmetric key based authentication, or public key cryptography, requires a deployment of a public key infrastructure (PKI). A TTP or Certificate Authority (CA) is needed to issue a signed certificate by binding entities' identities to their public keys. A small number of keys are sufficient for a large network. However, this approach is complex and requires high computation power and introduces communication overhead into the system. It is relatively expensive in terms of generating and verifying digital signatures which limit its practical application in cooperative wireless communication networks where the nodes have limited power and computational capability. In addition, the very nature of cooperative communication with its lack of fixed infrastructure makes it hard to interact through the TTP or to have a central repository for the digital certificates. The system throughput rate is comparatively smaller than the symmetric schemes. Commonly used asymmetric PKI algorithms include RSA [12], Rabin [13], Elliptic Curve Cryptography (ECC) [14], etc. Identity-Based Encryption (IBE) [15] is also a public-key encryption in which the public-key is a user's unique information, such as physical IP address. Asymmetric key with IBE approach is proposed to make key management easier through the use of a private key generator (PKG), which can be a TTP, generating a master public key known to public and a master secret key known only to the PKG. Encryption is carried out using the master public key and the recipient's ID. To decrypt, a private key is requested through a secure channel from a PKG after authenticating through a CA. The pair-wise shared key is used 
for authentication. As in the other asymmetric approach, this scheme is also easily scalable; and it is up to the receiver to obtain the private key to decrypt as only the public key is generated based on the ID. As the identities are pre-approved in the PKG to receive private keys, this scheme provides for implicit and non-interactive pre-authentication among the network nodes. This approach is not computationally resource intensive as compared to PKI. However, as the public key is not bound to a digital certificate, this can give rise to possible key escrow problem and in addition, key revocation can be challenging. Same as the PKI approach, this scheme is also not suitable for cooperative communication on account of lack of centralized control and the limited power and computational capability of the nodes. Commonly used asymmetric IBE schemes include Boneh/Franklin's pairing-based encryption scheme [16] and Cocks's encryption scheme [17].

For non-encryption based identity credential, information is hashed by using oneway hash function and the key processed by the supplicant. Thus, this method is computationally efficient. To verify the supplicant's identity, authenticator must own the key used by supplicant and know the one-way hash function used by supplicant to regenerate the results that were disclosed by supplicant as identity. Another form of hash based non-encryption identity credential uses delayed key disclosure as in TESLA [18], LHAP [19], HEAP [20] and ALPHA [21]. Timed efficient stream losstolerant authentication (TESLA) is a broadcast authentication protocol based on loose time synchronization. However, hop-by-hop authentication is not supported by TESLA. What is more, the computational overhead of TESLA is also high due to the existence of network latencies and redundant hash elements. A lightweight hopby-hop authentication protocol (LHAP) bases on the principles of TESLA to carry out both packet authentication and hop-by-hop authentication, wherein intermediate nodes authenticate all the packets received prior to forwarding them. However, LHAP 
also suffers from long latency and poor throughput, and is not designed to prevent inside attacks. Hop-by-hop efficient authentication protocol (HEAP) authenticates packets at every hop by using modified hash message authentication code (HMAC) based algorithm along with two keys and dropping any packet that originates from outsiders. However, HEAP still suffers from inside attack and could not provide end-to-end authentication. Adaptive and lightweight protocol for hop-by-hop authentication (ALPHA), which makes use of interaction-based hash chains and Merkle tress, provides both end-to-end and hop-by-hop authentication and integrity protection; overcomes the shortcomings of above proposed protocols. Therefore, ALPHA is adopted as the authentication protocol in the proposed game theoretic approach for security and QoS co-design in cooperative wireless communication networks. In the next section, ALPHA is described in details.

\subsection{ALPHA: An Adaptive and Lightweight Proto- col for Hop-by-hop Authentication}

ALPHA establishes a verifiable notion of identity for network traffic, based on hash chain [22] and enables end-to-end as well as hop-by-hop integrity protection. The basic idea behind hash chain [23] is to apply a cryptographic hash function $H$, i.e., SHA-1 [24], iteratively on a randomly generated seed $\theta$. The first result $H(\theta)=h_{1}$ is used as input for the next interaction, yielding $H(H(\theta))=H\left(h_{1}\right)=h_{2}$ until the hash chain has reached the desired length $i$. The last element of generated hash chain with desired length is called the anchor. Elements in the generated hash chain are used in a reverse sequence, i.e., starting with the anchor $h_{i}$ and proceeding with $h_{i-1}$. The nodes in cooperative wireless communication networks exchange their anchors through a secured channel for the purpose of identity authentication by disclosing the 
next hash chain element when an identity authentication is required.

Nodes in cooperative wireless communication networks act as source, relay and destination depending on the direction of packet flow. ALPHA supports bi-directional data flow. The source and the destination use distinct hash chains for signing outgoing and acknowledging incoming packets. Thus, the shared security context between the source and the destination consists of the respective anchors $\left\{h_{i}^{S s}, h_{i}^{S a}, h_{i}^{D s}, h_{i}^{D a}\right\}$. The two hash anchors with superscript $S$ are owned by the source while the other two anchors with superscript $D$ are owned by the destination. The source uses the first hash chain for signing the outgoing data; and the source uses the second hash chain for acknowledging the received data. The hash chains are denoted signature chain and acknowledgment chain by the seconded superscript $s$ and $a$ respectively. One pair of the source's signature chain and the destination's acknowledgment chain secures a simplex channel; the other pair of the source's acknowledgment chain and the destination's signature chain secures the other simplex channel. Therefore, the four-tuple secures a duplex-channel between the source and the destination.

\subsubsection{Basic ALPHA}

The basic ALPHA authentication scheme consists of a three-packet exchange process for each protected payload $m$ [25]. Figure 2 depicts the packet exchange process. The first packet sent from the source to the destination consists of a Message Authentication Code (MAC) $M$ of $m$ keyed with a fresh hash-chain element of the source. This packet fulfills three objectives. First, a fresh hash chain element $h_{i}^{S_{s}}$ of the source identifies the source. Second, an untampered MAC keyed with the source's next undisclosed signature chain element $M\left(h_{i}^{S_{s}}, m\right)$ ensures the integrity of $m$. Third, the sent packet works as trigger to the destination of sending an acknowledgment packet. In the second packet, the destination acknowledges the receipt of MAC; in the third 


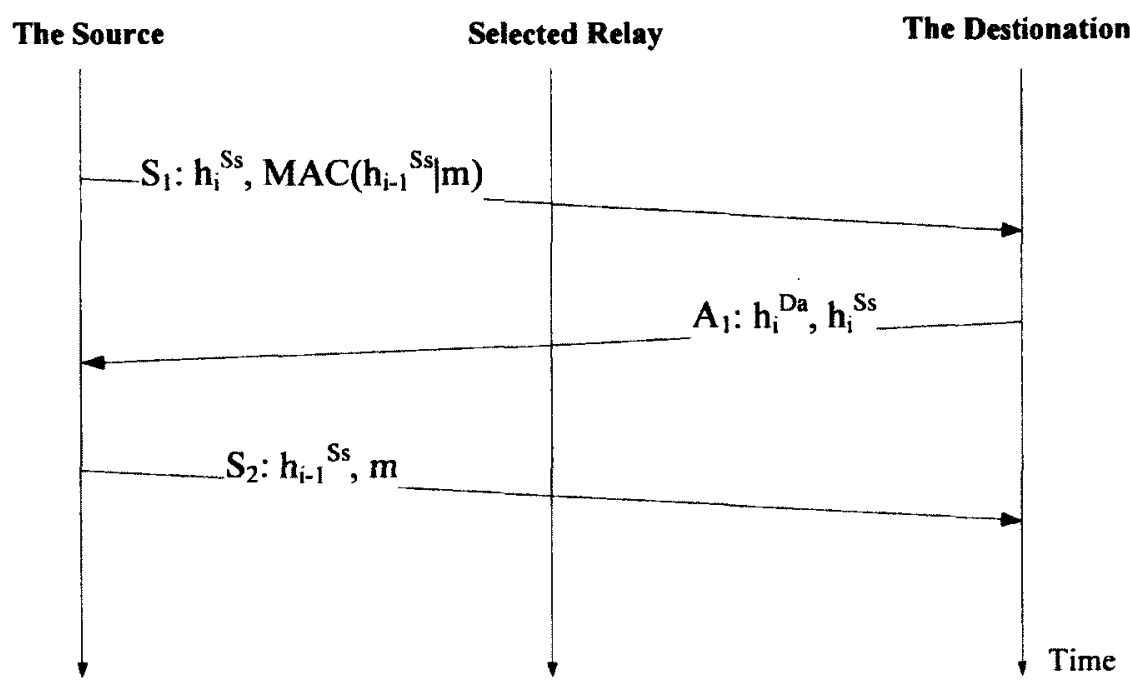

Figure 2: Basic ALPHA

packet, the source discloses the hash-chain element used as key of MAC and sends $m$. With this key, the destination and selected relay could check the integrity of $m$ by recalculating the received MAC.

\subsubsection{ALPHA-M: ALPHA-with Pre-signed Merkle Tree}

Support for transmitting a large amount of data is limited by basic ALPHA for its strict sequential packet exchange process. ALPHA-M provides an effective solution to ensure the verification of individual packet even though other packets are lost or arrive out of sequence due to the high packet loss rate and high possibility of out-of-order delivery in wireless communication networks.

ALPHA-M makes use of trees of hashes [26], which are called Merkle trees (MT), to generate pre-signatures and pre-acks/pre-nacks. A Merkle tree is a binary tree. Leaves of a Merkle tree consist of the hashes of the payloads and nodes in a Merkle tree consist of the hashes of its two children. Thus, the root of a Merkle tree depends on all the leaves. Any modification of the leaves results in a different root. Figure 3 


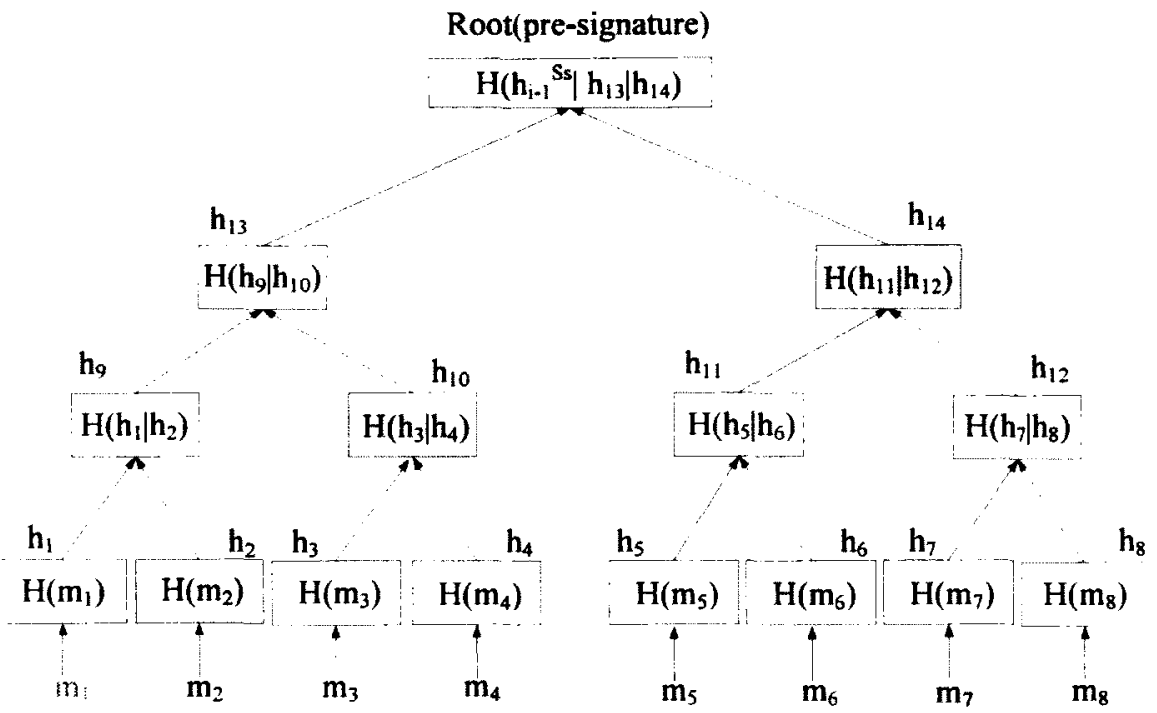

Figure 3: Merkle tree with 8 leaves

shows a typical structure of Merkle tree with 8 leaves.

Based on the characteristics of Merkle tree described above, each payload $m_{j}$ could be verified by an authenticator if following three messages are sent to the authenticator: the root, the hash value of payload $m_{j}$ and the set of complementary branches, which consists of the sibling nodes of the nodes on the path from the image of $m_{j}$ to the root of constructed Merkle tree. The authenticator calculates the tree root by using three received messages. Payload $m_{j}$ is authentic if and only if the root obtained through calculation matches the known root.

To selectively acknowledge every $m_{j}$, Merkle tree is also used by ALPHA-M for generating pre-acks/nacks. As shown in Figure 4, acks and nacks are placed in the leaves of an Acknowledgments Merkle tree. As shown in Figure 4, $2 n$ leaves are needed for acknowledging $n$ messages; $n$ leaves are for acks, the rest $n$ leaves are for n-acks. Each leaf of the Acknowledgments Merkle tree contains a secret $s_{i}$ and an index $x_{i}$, which identify the packet $m_{j}$. The secret in each leaf is different from the one in other leaf. The root is authenticated by the undisclosed hash chain element 


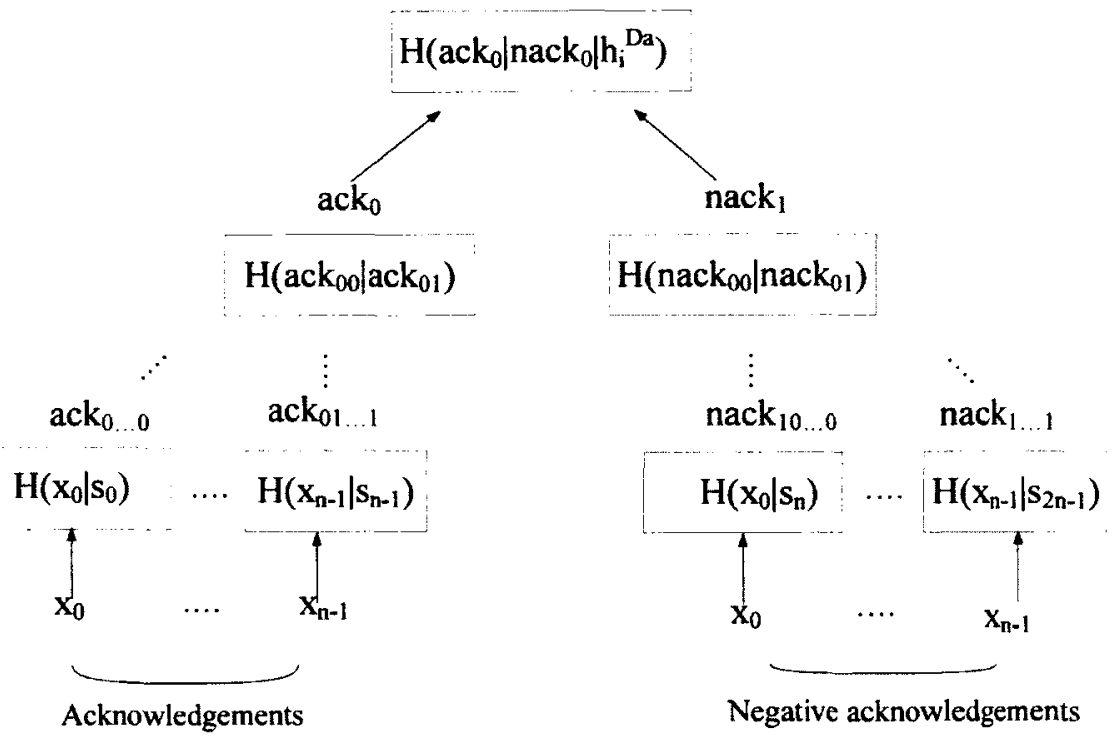

Figure 4: Acknowledgments Merkle tree

$h_{i-1}^{D_{a}}$.

As shown by Figure $5, A_{2}$ messages contains the ack/nack leaf whose pre-image is the index $x_{i}$ and secret $s_{i}$, and the set of Acknowledgments Merkle tree nodes $B_{c}^{D_{a}}$ that is used to compute root to verify the destination's identity. Each relay can also verify each acknowledgment sent by the destination by buffering $A_{1}$ messages.

\subsection{Game Theory}

Game theory is a discipline used to model situations in which decision makers have to make specific actions that have mutual, possibly conflicting interests. It has been used primarily in economics, in order to model competition between companies, e.g., should a given company enter a new market or not, considering that its competitors could make similar or different moves? Game theory has also been applied to other areas, including politics and biology. The first textbook in this area was written by John von Neumann and Oskar Morgenstern [27]. A few years later, John Nash 


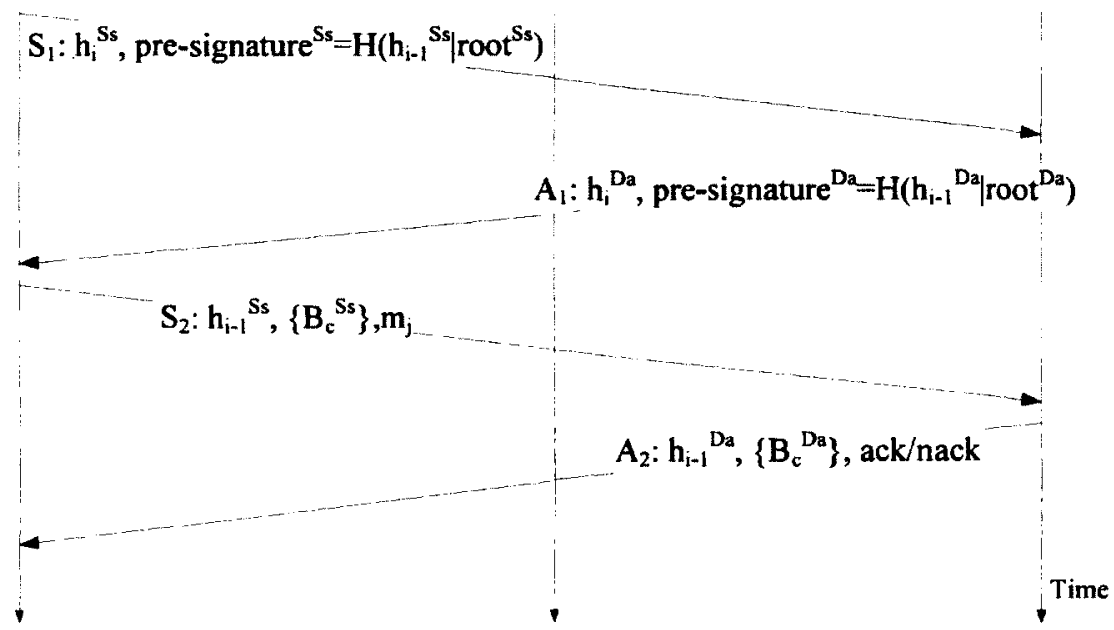

Figure 5: ALPHA-M with Pre-(n)acks

made a number of additional contributions, the cornerstone of which was the famous Nash equilibrium [28]. Since then, many other researchers have contributed to game theory [29].

Game theory has also been applied to networking to solve the problems concerning resource allocation and routing in a competitive phenomenon [30]. In the past few years, game theory was also applied to wireless communication networks. Players in the game have to cope with limited system resources, such as constrained battery life and limited computing capacity that impose a conflict of interests that each player in the game tries to maximize its utility with minimum cost. To keep consistent with the practice of game theory, we assume that all the players are rational, which means that all of players try their best to maximize their utility. Our assumption on the rationality of players is reasonable, because most interactions in wireless communication networks could be captured by using the concept of rationality with appropriate adjustment of the utility function. To maximize their utility, the players act according to their competitors' strategies [31]. 


\subsubsection{Static Game and Dynamic Bayesian Game}

A game consists of three components, set of players, set of players' strategies and set of players' utility functions. In the proposed game theoretic approach for security and QoS co-design in cooperative wireless communication networks, we assume that there are two players, the attacker and the source. The attacker selects attacking target from all candidate relays and tries its best to compromise intermediate relays; while the source adopts a strategic approach on relay selection. The set of players' strategies, which is composed by all players' pure strategies. Players in game could not only take pure strategy by assigning probability 0 to all the strategies in its strategy set but 1 to the strategy it takes; but also take mixed strategies by assigning different probabilities to all strategies. In the proposed static game theoretic approach, we assume that all the relays are attacker's attacking targets, thus attacker's strategy set consists of strategies: Attack and Not attack; the source's strategy set consists of strategies: Select and Not select. Set of players' functions is made of the utility functions for all game players, which takes the strategic actions of players as input.

In game-theoretic terms, a static game is the one in which all players make decisions or select strategies simultaneously, without the knowledge of the strategies that are being adopted by other players. Even though the decisions may be made at different points in time, the game is simultaneous because each player has no information about the decisions of others; it is as if the decisions are made simultaneously. Therefore, static game can be also viewed as a game of imperfect information.

Contrary to the static game in which all the players choose their strategies simultaneously, players interact in dynamic game by playing a similar stage game for numerous times [32] [33]. In cooperative wireless communication networks, there exist cooperative relays that are not attacked and compromised by the attacker and thus never intend to perform negatively during the data transmission process. Therefore, 
assumption made in the proposed static game theoretic approach for security and QoS co-design in cooperative wireless communication networks that all the relays are malicious is not accurate anymore. To more accurately depict the interactions between the attacker and the source, we propose a dynamic Bayesian game theoretic approach for security and QoS co-design in cooperative wireless communication networks [34]. Players in a dynamic Bayesian game have initial beliefs about the type of each player. Belief is a probability distribution over the possible type of a player and can be updated according to Bayes' rule as play takes place in the game, i.e., the belief a player holds about another player's type might change on the basis of the actions they have played. In the proposed dynamic Bayesian game theoretic approach, the source is not sure about the types of relays, thus the source assigns prior beliefs on the types of relays and updates its beliefs on relays based on the detected attacker's action histories on relays by using Bayes' rule.

According to John C. Harsanyi's work [35], a Bayesian game can be modeled by introducing Nature as a player in a game. Nature assigns a random variable to each player which could take values of types for each player and associates probabilities or probability density functions with those types. In the proposed dynamic Bayesian game theoretic approach for security and QoS co-design in cooperative wireless communication networks, we take the type of each relay into consideration which is not considered by the proposed static approach that assumes all candidate relays are completely malicious. In dynamic game theoretic approach, we suppose that there exist two possible types for each relay, malicious and cooperative. Detailed description of the proposed static and dynamic Bayesian game theoretic approach will be made in the next chapter. 


\subsection{Summary}

In this chapter, first of all, fundamental concepts concerning cooperative wireless communication networks, signaling schemes and opportunistic relaying are presented. Secondly, the security issues that arise in cooperative wireless communication networks due to its decentralized organization and proposed approaches for combating security problems in cooperative wireless communication networks are discussed. Finally, application of game theoretic approach for security and QoS co-design is bricfly described. In the next chapter, the proposed game theoretic approach for security and QoS co-design in cooperative wireless communication networks is presented in details. 


\section{Chapter 3}

\section{Proposed Game Theoretic Approach}

In this chapter, the proposed game theoretic approach for security and QoS co-design in cooperative wireless communication networks is described in details by setting up the system model, and presenting the utility of the attacker brought by attacking target selection and the utility of the source brought by relay selection, Nash equilibrium of the proposed game theoretic approach and equations of system performance analysis.

\subsection{Model Description}

The proposed game theoretic approach focuses on two-hop cooperative wireless communication networks, as illustrated in Figure 6, consisting of source, destination, four intermediate relays and a slow-fading channel that satisfies Rayleigh distribution. All the relays are originally assumed to be cooperative and the selected relay forwards the received information from the source to the destination. But in reality, some relays are compromised by the attacker and do not do what they are supposed to do or do what they are not supposed to do. 


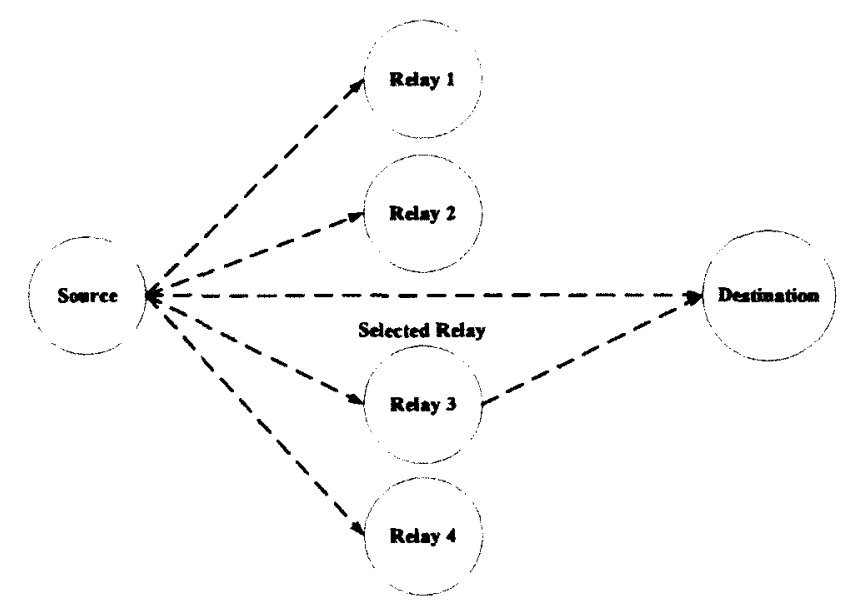

Figure 6: A cooperative wireless communication network

\subsection{Game Played for Security and QoS Co-Design}

In this thesis, we represent the set of relays as $\mathcal{R}$. Attack on relays initiated by the attacker is independent with each other. The interactions between the attacker and the source are modeled as a noncooperative game, since both the tendencies of the attacker and the source are to maximize their total utility through the strategic selection of attacking target and relay. The attacker selects the attacking probability distribution $P=\left\{p_{1}, p_{2}, \ldots, p_{K}\right\}$ over all relays in $\mathcal{R}$, where $p_{i}$ is the probability of selecting $R_{i}$ as attacking target, $K$ is the number of candidate relays in the radio coverage of the source. In each play of the game, the attacker chooses one relay to attack; thus, we have $\sum_{i}^{K} p_{i}=1$. For the source, it selects all candidate relays with a probability distribution $Q=\left\{q_{1}, q_{2}, \ldots, q_{K}\right\}$, where $q_{i}$ is the probability of selecting $R_{i}$ as relay. In each play of the game, the source chooses one relay from all candidate relays; thus, we have $\sum_{i}^{K} q_{i}=1$. We assume that each relay processes a combination of information and security assets denoted by $\alpha_{I} I_{i}+\alpha_{S} S_{i}, i \in 1,2, \ldots, K$, which represents the loss of information and security assets when attacker's attacking target selection coincides the source's relay selection. $\alpha_{I}$ and $\alpha_{S}$ represent the weights 
of information and security assets in the asset combination. The information asset of a relay depends on the mutual information, while the security asset of a relay depends on its role in the network. In practice, the information asset is evaluated by the mutual information which affects the system throughput of cooperative wireless communication networks; and the security asset is evaluated in the risk analysis by using formal analysis before system deployment.

\subsubsection{Static Game Theoretic Approach}

In static game theoretic approach, we assume that on each relay, the attacker takes two actions, choose to attack, denoted as Attack or does not choose to attack, denoted as Not attack; similarly, on each relay, the source takes two actions, Select and Not select. This assumption applies to model the networks in which all relays have been suffering from constant attack initiated by the attacker. However, there actually exist such networks in which not all candidate relays are not attacked by the attacker. We consider these relays as cooperative. Therefore, assumption made in static game theoretic approach now seems an overemphasis on the vulnerability of the system to potential attack, which leads to unnecessary execution of encryption and authentication protocols and results in waste of limited computing resource when cooperative/trustworthy relays are selected. Therefore, we propose a dynamic approach under the inspiration of Bayesian game, by which the source's belief in type of each relay, malicious or cooperative, is updated at the end of each round of game played based on attacker's attacking histories on selected relays. The proposed static game theoretic approach can be considered as the special case of the proposed dynamic game theoretic approach, in which the source's beliefs in the maliciousness of all relays are complete and constant. 
Table 3: Utility matrix of attacker and source on relay $R_{i}$

\begin{tabular}{|c|c|c|}
\hline & Select & Not select \\
\hline Attack & $\left(1-2 a-C_{a}\right) A_{i},-\left(1-2 a+C_{m}\right) A_{i}$ & $\left(1-C_{a}\right) A_{i},-A_{i}$ \\
\hline Not attack & $0,-\left(b C_{f}+C_{m}\right) A_{i}$ & 0,0 \\
\hline
\end{tabular}

In static game theoretic approach, if relay $R_{i}$ is selected by the attacker as attacking target and selected by the source as relay as well, then the attacker will obtain utility $\alpha_{I} I_{i}+\alpha_{S} S_{i}$, while the source will lose the same amount of utility. Otherwise, the utility for the attacker and the source are $-\left(\alpha_{I} I_{i}+\alpha_{S} S_{i}\right)$ and $\alpha_{I} I_{i}+\alpha_{S} S_{i}$, respectively. We have to note that other types of utility formulations are also possible. In those cases, analysis in this thesis can be extended by modifying the utility functions of the attacker and the source. Substitute $\alpha_{I} I_{i}+\alpha_{S} S_{i}$ by $A_{i}$, Table 1 illustrates the utility matrix of the attacker and the source on relay $R_{i}$ in the strategic form. In the matrix, $a$ denotes the attacking detection rate of the source, $b$ denotes the false alarm rate, and $0 \leq a, b \leq 1$. The cost of attacking for attacker and attack monitoring for source, $C_{a}$ and $C_{m}$, are taken into consideration in our model and assumed proportional to the value set of relay $R_{i}$, denoted by $C_{a}\left(\alpha_{I} I_{i}+\alpha_{S} S_{i}\right)$ and $C_{m}\left(\alpha_{I} I_{i}+\alpha_{S} S_{i}\right)$. $C_{f}\left(\alpha_{I} I_{i}+\alpha_{S} S_{i}\right)$ denotes the loss of source caused by false alarm [36] [37].

We denote the total utility of the attacker and the source as $U_{A}(P, Q)$ and $U_{S}(P, Q):$

$$
\begin{aligned}
U_{A}(P, Q) & =\sum_{i \in \mathcal{R}} p_{i} q_{i}\left(1-2 a-C_{a}\right) A_{i}+p_{i}\left(1-q_{i}\right)\left(1-C_{a}\right) A_{i} \\
& =\sum_{i \in \mathcal{R}} p_{i} A_{i}\left(1-2 a q_{i}-C_{a}\right)
\end{aligned}
$$




$$
\begin{aligned}
U_{S}(P, Q) & =\sum_{i \in \mathcal{R}}-p_{i} q_{i}\left(1-2 a+C_{m}\right) A_{i}-p_{i}\left(1-q_{i}\right) A_{i} \\
& -\left(1-p_{i}\right) q_{i}\left(b C_{f}+C_{m}\right) A_{i} \\
& =\sum_{i \in \mathcal{R}} q_{i} A_{i}\left[p_{i}\left(2 a+b C_{f}\right)-\left(b C_{f}+C_{m}\right)\right]-p_{i} A_{i}
\end{aligned}
$$

\subsubsection{Dynamic Bayesian Game Theoretic Approach}

The proposed dynamic Bayesian game theoretic approach also consists of two players, the source which selects the best relay from all candidate relays that brings maximum utility and the attacker which selects relay as attacking target. The set of strategies of the source contains Select and Not select. Attack and Not attack consist of the attacker's strategies on relay $R_{i}$ when attacker may choose relay $R_{i}$ to attack; otherwise, there is only one strategy when attacker does not choose relay $R_{i}$ as attacking target, i.e., Not attack. Since the source is uncertain about the type of each relay, it holds a priori belief $\mu_{i}^{t_{k}}, i=\{1,2, \ldots, K\}$ in the maliciousness of relay $R_{i}$ at the commence of each relay selection stage $t_{k}, k=\{1,2, \ldots\} .1-\mu_{i}^{t_{k}}, i=\{1,2, \ldots, K\}$, $k=\{1,2, \ldots\}$, represents the source's prior belief in that relay $R_{i}$ is cooperative. We assume that the game in the proposed dynamic game theoretic approach is played repeatedly every period $t_{k}$, where $k=0,1 \ldots$ We assume that the utility of players in each stage remain the same. We assume that each relay node processes a combination of information and security assets denoted by $\alpha_{I} I_{i}+\alpha_{S} S_{i} . \alpha_{I}$ and $\alpha_{S}$ represent the weights of information and security assets in the asset combination and vary in various networks.

If the selection of relay of the source and the selection of attacking target of the attacker coincide, the attacker will obtain utility $\alpha_{I} I_{i}+\alpha_{S} S_{i}$, while the source will lose the same amount of utility. Otherwise, the utility for the attacker and the source is $-\left(\alpha_{I} I_{i}+\alpha_{S} S_{i}\right)$ and $\alpha_{I} I_{i}+\alpha_{S} S_{i}$, respectively. Substitute $\alpha_{I} I_{i}+\alpha_{S} S_{i}$ by $A_{i}$, Table 
Table 4: Utility matrix of attacker and source on relay $R_{i}$ with probability $\mu_{i}^{t_{k}}$ being malicious at stage $t_{k}$

\begin{tabular}{|c|c|c|}
\hline & Attack & Not attack \\
\hline Select & $-\left(1-2 a-C_{a}\right) A_{i},\left(1-2 a-C_{a}\right) A_{i}$ & $-\left(b C_{f}+C_{m}\right) A_{i}, 0$ \\
\hline Not select & $-A_{i},\left(1-C_{a}\right) A_{i}$ & 0,0 \\
\hline
\end{tabular}

Table 5: Utility matrix of attacker and source on relay $R_{i}$ with probability $1-\mu_{i}^{t_{k}}$ being cooperative at stage $t_{k}$

\begin{tabular}{|c|c|}
\hline & Not attack \\
\hline Select & $-\left(b C_{f}+C_{m}\right) A_{i}, 0$ \\
\hline Not select & 0,0 \\
\hline
\end{tabular}

4 illustrates the utility matrix of attacker and source on relay $R_{i}$ with probability $\mu_{i}^{t_{k}}$ being malicious at stage $t_{k}$. In the matrix, $a$ denotes the detection rate of the source, $b$ denotes the false alarm rate, and $0 \leq a, b \leq 1$. The cost of attacking for malicious node and monitoring for the source, $C_{a}$ and $C_{m}$, are taken into consideration in our model and assumed proportional to the value set of relay $R_{i}$, denoted by $C_{a}\left(\alpha_{I} I_{i}+\alpha_{S} S_{i}\right)$ and $C_{m}\left(\alpha_{I} I_{i}+\alpha_{S} S_{i}\right) . C_{f}\left(\alpha_{I} I_{i}+\alpha_{S} S_{i}\right)$ denotes the loss of the source caused by false alarm. Table 5 illustrates the utility matrix of attacker and source on relay $R_{i}$ with probability $1-\mu_{i}^{t_{k}}$ being cooperative at stage $t_{k}$.

\subsubsection{Bayesian Updating Rule on Beliefs in the Maliciousness of Relays}

In this section, we define a Bayesian updating rule on beliefs in the maliciousness of relays, which is based on the source's initial beliefs and the source's record of attacker's attacking histories on relays [38] [39].

For a given relay $R_{i}$, we define a sequence of random variables $T_{i}^{t_{1}}, T_{i}^{t_{2}}, \ldots$, where $T_{i}^{t_{k}}$ characterizes the belief in the cooperativeness of relay $R_{i}$ at stage $t_{k}$. For instance, 
suppose that at stage $t_{k}, M_{i}^{t_{k}}$ packets have been sent by the source through selected relay $R_{i}$ to the destination. Let $N_{i}^{t_{k}}$ be the number of packets successfully forwarded by the selected relay $R_{i}$ to the destination, out of the $M_{i}^{t_{k}}$ packets sent to selected relay $R_{i}$ for forwarding at stage $t_{k}$. Suppose a prior probability density function for $T_{i}^{t_{k-1}}$, denoted by $f_{i}^{t_{k-1}}(m, n, t)$, is known. The posterior probability density function $f_{i}^{t_{k}}(m, n, t)$, given the number of received packets $M_{i}^{t_{k}}$ and forwarded packets $N_{i}^{t_{k}}$, can be obtained as follows:

$$
f_{i}^{t_{k}}(m, n, t)=\frac{f_{i}^{t_{k}}\left(N_{i}^{t_{k}}=n \mid t, M_{i}^{t_{k}}=m\right) f_{i}^{t_{k-1}}(m, n, t)}{\int_{0}^{1} f_{i}^{t_{k}}\left(N_{i}^{t_{k}}=n \mid \imath, M_{i}^{t_{k}}=m\right) f_{i}^{t_{k-1}}(m, n, t) d t}
$$

where $f_{i}^{t_{k}}\left(N_{i}^{t_{k}}=n \mid t, M_{i}^{t_{k}}=m\right)$ is called the like-hood function and defined as follows:

$$
f_{i}^{t_{k}}\left(N_{i}^{t_{k}}=n \mid t, M_{i}^{t_{k}}=m\right)=\left(\begin{array}{c}
m \\
n
\end{array}\right) t^{n}(1-t)^{m-n}
$$

It can be shown that the posterior probability density function $f_{i}^{t_{k}}(m, n, t)$ follows a Beta distribution. The Beta distribution with parameters $a$ and $b$ is defined as follows:

$$
\operatorname{Beta}(a, b)=\frac{t^{a-1}(1-t)^{b-1}}{B(a, b)}=\frac{t^{a-1}(1-t)^{b-1}}{\int_{0}^{1} t^{a-1}(1-t) d t}
$$

for $0 \leq t \leq 1$. In particular, if

$$
f_{i}^{t_{k-1}}(m, n, t) \sim \operatorname{Beta}\left(a_{i}^{t_{k-1}}, b_{i}^{t_{k-1}}\right),
$$

then given that $M_{i}^{t_{k}}=m_{i}^{t_{k}}$ and $N_{i}^{t_{k}}=n_{i}^{t_{k}}$, we have

$$
f_{i}^{t_{k}}(m, n, t) \sim \operatorname{Beta}\left(a_{i}^{t_{k-1}}+n_{i}^{t_{k}}, b_{i}^{t_{k-1}}+m_{i}^{t_{k}}-n_{i}^{t_{k}}\right) .
$$


Therefore, $f_{i}^{t_{k}}(m, n, t)$ is characterized by the parameters $a_{i}^{t_{k}}$ and $b_{i}^{t_{k}}$, which is defined recursively as follows:

$$
a_{i}^{t_{k}}=a_{i}^{t_{k-1}}+n_{i}^{t_{k}}
$$

and

$$
b_{i}^{t_{k}}=b_{i}^{t_{k-1}}+m_{i}^{t_{k}}-n_{i}^{t_{k}} .
$$

Therefore, belief in the maliciousness of relay $R_{i}$ at stage $t_{k}$ is

$$
\mu_{i}^{t_{k}}=1-f_{i}^{t_{k}}(m, n, t)
$$

which could be calculated recursively through the record of $a_{i}$ and $b_{i}[40]$.

At the system initial stage $t_{0}$, there is no information for the cooperative wireless communication networks. Therefore, we assume that $T_{i}^{t_{0}}$ has the uniform distribution over the interval $[0,1]$, i.e.,

$$
f_{i}^{t_{0}}(m, n, t) \sim U[0,1]=\operatorname{Beta}(1,1),
$$

which indicates the source's indifference to the selected relay's behavior at stage $t_{0}$.

\subsection{Find Nash Equilibrium of the Proposed Game Theoretic Approach}

In cooperative wireless communication networks, both the attacker and the source have limited system resources, such as limited battery life and limited computational capacity; it is natural for the attacker to focus on attacking some targets that are 
more beneficial compared by initiating attack on the others. Same to [36], we sort the targets according to their combination of information and security assets and divide the whole target set into three subsets, sensible target set, quasi-sensible and non-sensible target set according to the weight of each relay's asset over the overall assets composed by all relays' that belong to $\mathcal{R}$.

Definition 3.3.1 The sensible target set $\mathcal{R}_{S}$, the quasi-sensible target set $\mathcal{R}_{Q}$ and non-sensible target set $\mathcal{R}_{N}$ are defined as follows:

$$
\begin{cases}\alpha_{I} I_{i}+\alpha_{S} S_{i}>\frac{\left|\mathcal{R}_{S}\right|\left(1-C_{a}\right)-2 a}{\left(1-C_{a}\right) \sum_{j \in \mathcal{R}_{s}} \overline{\alpha_{I} I_{j}+\alpha_{S} S_{j}}}, & \forall i \in \mathcal{R}_{S} \\ \alpha_{I} I_{i}+\alpha_{S} S_{i}=\frac{\left|\mathcal{R}_{S}\right|\left(1-C_{a}\right)-2 a}{\left(1-C_{a}\right) \sum_{j \in \mathcal{R}_{s}} \overline{\alpha_{\alpha_{I}} I_{j}+\alpha_{S} S_{j}}}, & \forall i \in \mathcal{R}_{Q} \\ \alpha_{I} I_{i}+\alpha_{S} S_{i}<\frac{\left|\mathcal{R}_{S}\right|\left(1-C_{a}\right)-2 a}{\left(1-C_{a}\right) \sum_{j \in \mathcal{R}_{s}} \overline{\alpha_{I} I_{j}+\alpha_{S} S_{j}}}, & \forall i \in \mathcal{R}_{N}\end{cases}
$$

where $\left|\mathcal{R}_{S}\right|$ is the cardinality of $\mathcal{R}_{S}$.

The cardinality of $\mathcal{R}_{S}$ could be calculated as follows:

1. if $\alpha_{I} I_{K}+\alpha_{S} S_{K}>\frac{K\left(1-C_{a}\right)-2 a}{\left(1-C_{a}\right) \sum_{j=1}^{\mid R} \frac{1}{\alpha_{I} I_{j}+\alpha_{S} S_{j}}}$, then $\left|\mathcal{R}_{S}\right|=K$ and $\left|\mathcal{R}_{Q}\right|=0$.

2. if $\alpha_{I} I_{K}+\alpha_{S} S_{K} \leq \frac{K\left(1-C_{a}\right)-2 a}{\left(1-C_{a}\right) \sum_{j=1}^{|\mathcal{R}|} \frac{1}{\alpha_{I} I_{j}+\alpha_{S} S_{j}}},\left|\mathcal{R}_{S}\right|$ is determined by the following formulas:

$$
\left\{\begin{array}{l}
\alpha_{I} I_{\left|\mathcal{R}_{S}\right|}+\alpha_{S} S_{\left|\mathcal{R}_{S}\right|}>\frac{\left|\mathcal{R}_{S}\right|\left(1-C_{a}\right)-2 a}{\left(1-C_{a}\right) \sum_{j=1}^{\left|\mathcal{R}_{S}\right|} \frac{1}{\alpha_{I} I_{j}+\alpha_{S} S_{j}}} \\
\alpha_{I} I_{\left|\mathcal{R}_{S}\right|+1}+\alpha_{S} S_{\left|\mathcal{R}_{S}\right|+1} \leq \frac{\left|\mathcal{R}_{S}\right|\left(1-C_{a}\right)-2 a}{\left(1-C_{a}\right) \sum_{j=1}^{\left|\mathcal{R}_{S}\right|} \frac{1}{\alpha_{I} I_{j}+\alpha_{S} S_{j}}}
\end{array}\right.
$$

Quasi-sensible target set $\mathcal{R}_{Q}$ consists of relay nodes whose assets are equal to

$$
\frac{\left|\mathcal{R}_{S}\right|\left(1-C_{a}\right)-2 a}{\left(1-C_{a}\right) \sum_{j=1}^{\left|\mathcal{R}_{S}\right|} \frac{1}{\alpha_{I} I_{j}+\alpha_{S} S_{j}}}
$$


Theorem 1 in [36] shows that a rational attacker will not attack any relay that belongs to the non-sensible set, because the utility obtained by successful attack on these relays in non-sensible set is negligible compared with the utility obtained on these with higher asset combinations.

\subsubsection{Find Nash Equilibrium of the Proposed Static Game Theoretic Approach}

As mentioned above, there are two players in the proposed static game theoretic approach, and thus there exists at least one Nash equilibrium [41] [42]. It holds that

$$
\begin{aligned}
& 0 \leq\left(1-2 a q_{i}^{*}-C_{a}\right) A_{i}=\left(1-2 a q_{j}^{*}-C_{a}\right) A_{j} \geq\left(1-2 a q_{k}^{*}-C_{a}\right) A_{k} \\
& \forall i, j, k \in \mathcal{R}, p_{i}^{*}, p_{j}^{*}>0, p_{k}^{*}=0,
\end{aligned}
$$

which can be shown by noticing the attacker's total utility function $U_{A}(P, Q)$ : if $\left(1-2 a q_{i}^{*}-C_{a}\right) A_{i}<0$, then the attacker has incentive to change $p_{i}^{*}$ to 0 ; if $\left(1-2 a q_{i}^{*}-\right.$ $\left.C_{a}\right) A_{i}<\left(1-2 a q_{j}^{*}-C_{a}\right) A_{j}$, then the attacker is inclined to decrease $p_{i}^{*}$ and increase $p_{j}^{*}$; if $\left(1-2 a q_{j}^{*}-C_{a}\right) A_{j}<\left(1-2 a q_{k}^{*}-C_{a}\right) A_{k}$, then the attacker obtains more utility by adding $p_{i}^{*}$ to $p_{k}^{*}$ and setting $p_{i}^{*}$ equal to 0 . Similarly, noticing the source's total utility function $U_{S}(P, Q)$, it holds that

$$
\begin{array}{rc}
0 \leq A_{i}\left[p_{i}^{*}\left(2 a+b C_{f}\right)-\left(b C_{f}+C_{m}\right)\right]=A_{j}\left[p_{j}^{*}\left(2 a+b C_{f}\right)-\left(b C_{f}+C_{m}\right)\right] & \\
\geq A_{k}\left[p_{k}^{*}\left(2 a+b C_{f}\right)-\left(b C_{f}+C_{m}\right)\right] & \forall i, j, k \in \mathcal{R}, q_{i}^{*}, q_{j}^{*}>0, q_{k}^{*}=0 .
\end{array}
$$

To obtain the Nash equilibrium $\left(P^{*}, Q^{*}\right)$ of the proposed static game theoretic approach, we need to reclaim that $A_{i}>A_{j}$, if $i>j$ and $\sum_{i}^{|\mathcal{R}|} p_{i}^{*}=\sum_{i}^{|\mathcal{R}|} q_{i}^{*}=1$. 
From

$$
\begin{aligned}
& \left(1-2 a q_{i}^{*}-C_{a}\right) A_{i}=\left(1-2 a q_{j}^{*}-C_{a}\right) A_{j} \\
& A_{i}\left[p_{i}^{*}\left(2 a+b C_{f}\right)-\left(b C_{f}+C_{m}\right)\right]=A_{j}\left[p_{j}^{*}\left(2 a+b C_{f}\right)-\left(b C_{f}+C_{m}\right)\right]
\end{aligned}
$$

we have

$$
\begin{aligned}
p_{i}^{*} & =\frac{1}{2 a+b C_{f}}\left\{b C_{f}+C_{m}+\frac{A_{j}}{A_{i}}\left[p_{j}^{*}\left(2 a+b C_{f}\right)-\left(b C_{f}+C_{m}\right]\right\}\right. \\
q_{i}^{*} & =\frac{1}{2 a}\left[1-C_{a}-\frac{\left(1-2 a q_{j}^{*}-C_{a}\right) A_{j}}{A_{i}}\right] .
\end{aligned}
$$

Following are components of the Nash equilibrium $\left(P^{*}, Q^{*}\right)$ of the proposed static game theoretic approach:

$$
\begin{aligned}
& p_{i}^{*} \begin{cases}=\frac{1}{A_{i} \sum_{j=1}^{\mid \mathcal{R}} \frac{1}{A_{j}}}-\left(\frac{\left|\mathcal{R}_{S}\right|}{A_{i} \sum_{j=1}^{|R|} \frac{1}{A_{j}}}\right) \cdot \frac{b C_{f}+C_{m}}{2 a+b C_{f}}, & i \in \mathcal{R}_{S} \\
\in\left[0, \frac{1}{A_{i} \sum_{j=1}^{|\mathcal{R}|} \frac{1}{A_{j}}}-\left(\frac{\left|\mathcal{R}_{S}\right|}{A_{i} \sum_{j=1}^{|\mathcal{R}|} \frac{1}{A_{j}}}\right) \cdot \frac{b C_{f}+C_{m}}{2 a+b C_{f}}\right], & i \in \mathcal{R}_{Q} \\
=0, & i \in \mathcal{R}_{N}\end{cases} \\
& q_{i}^{*}= \begin{cases}\frac{1}{2 a}\left(1-C_{a}-\frac{\left|\mathcal{R}_{S}\right|\left(1-C_{a}\right)-2 a}{A_{i} \sum_{j=1}^{|\mathcal{R}|} \frac{1}{A_{j}}}\right), & i \in \mathcal{R}_{S} \\
0, & \text { otherwise }\end{cases}
\end{aligned}
$$

where

$$
\sum_{i \in \mathcal{R}} p_{i}^{*}=\sum_{i \in \mathcal{R}} q_{i}^{*}=1
$$


Table 6: Utility matrix of attacker and source on relay $R_{i}$ at stage $t_{k}$ after Harsanyi transformation

\begin{tabular}{|c|c|c|}
\hline & Attack, Not attack* & Not attack, Not attack* \\
\hline Select & $\begin{array}{c}-\mu_{i}^{t_{k}}\left(1-2 a+C_{m}\right) A_{i}-\left(1-\mu_{i}^{t_{k}}\right)\left(b C_{f}+C_{m}\right) A_{i}, \\
\mu_{i}^{t_{k}}\left(1-2 a-C_{a}\right) A_{i}\end{array}$ & $-\left(b C_{f}+C_{m}\right) A_{i}, 0$ \\
\hline Not select & $-\mu_{i}^{t_{k}} A_{i}, \mu_{i}^{t_{k}}\left(1-C_{a}\right) A_{i}$ & 0,0 \\
\hline
\end{tabular}

\subsubsection{Find Nash Equilibrium of the Proposed Dynamic Game Theoretic Approach}

Up to now, we have obtained two players' strategies on all candidate relays in the form of attacking and selection probabilities for the proposed static game theoretic approach. The first step in finding the Nash equilibrium of the proposed dynamic Bayesian game theoretic approach used for modeling the interactions between the source and the attacker is to apply Harsanyi transformation that converts the incomplete information game into a normal form game. Given that the Harsanyi transformation is a standard concept in game theory, we introduce it literally without introducing mathematical formula concerning Harsanyi transformation [43]. For each relay, there are two possible types, malicious with probability $\mu_{i}^{t_{k}}$ and cooperative with probability $1-\mu_{i}^{t_{k}}$. We combine the utility matrix of Table 4 and the utility matrix of Table 5 to obtain Table 6 whose components are expected utilities of malicious type relay and cooperative type relay. There are two combined attacking strategies for the attacker, Attack, Not attack* and Not attack and Not attack*, in which Not attack* is the pure strategy of the attacker on cooperative relay.

Denote the total utility for the attacker and the source by $U_{A}^{t_{k}}(P, Q)$ and $U_{S}^{t_{k}}(P, Q)$ 
at stage $t_{k}$ as:

$$
\begin{aligned}
U_{A}^{t_{k}}(P, Q)= & \sum_{i \in \mathcal{R}} p_{i} q_{i} \mu_{i}^{t_{k}}\left[(1-2 a) A_{i}-C_{a} A_{i}\right]+p_{i}\left(1-q_{i}\right) \mu_{i}^{t_{k}}\left(1-C_{a}\right) A_{i} \\
= & \sum_{i \in \mathcal{R}} p_{i} A_{i} \mu_{i}^{t_{k}}\left(1-2 a q_{i}-C_{a}\right) \\
U_{S}^{t_{k}}(P, Q)= & \sum_{i \in \mathcal{R}} p_{i} q_{i}\left[-\mu_{i}^{t_{k}}\left(1-2 a+C_{m}\right) A_{i}-\left(1-\mu_{i}^{t_{k}}\right)\left(b C_{f}+C_{m}\right) A_{i}\right] \\
& -p_{i}\left(1-q_{i}\right) \mu_{i}^{t_{k}} A_{i}-\left(1-p_{i}\right) q_{i}\left(b C_{f}+C_{m}\right) A_{i} \\
= & \sum_{i \in \mathcal{R}} q_{i} A_{i}\left[p_{i} \mu_{i}^{t_{k}}\left(2 a+b C_{f}\right)-\left(b C_{f}+C_{m}\right)\right]-p_{i} \mu_{i}^{t_{k}} A_{i}
\end{aligned}
$$

The attacker and the source select their strategies $P^{*}$ and $Q^{*}$ to maximize $U_{A}^{t_{k}}(P, Q)$ and $U_{S}^{t_{k}}(P, Q)$.

Similar to the Nash equilibrium obtained from the proposed static game theoretic approach, it holds that

$$
\begin{aligned}
& 0 \leq\left(1-2 a q_{i}^{*}-C_{a}\right) A_{i} \mu_{i}^{t_{k}}=\left(1-2 a q_{j}^{*}-C_{a}\right) A_{j} \mu_{j}^{t_{k}} \\
& \geq\left(1-2 a q_{k}^{*}-C_{a}\right) A_{k} \mu_{k}^{t_{k}}
\end{aligned}
$$

$$
\forall i, j, k \in \mathcal{R}, p_{i}^{*}, p_{j}^{*}>0, p_{k}^{*}=0
$$

which can be shown by noticing the attacker's total utility function $U_{A}^{t_{k}}(P, Q)$ : if $\left(1-2 a q_{i}^{*}-C_{a}\right) A_{i} \mu_{i}^{t_{k}}<0$, then the attacker has incentive to change $p_{i}^{*}$ to 0 ; if $\left(1-2 a q_{i}^{*}-C_{a}\right) A_{i} \mu_{i}^{t_{k}}<\left(1-2 a q_{j}^{*}-C_{a}\right) A_{j} \mu_{j}^{t_{k}}$, then the attacker is inclined to decrease $p_{i}^{*}$ and increase $p_{j}^{*}$; if $\left(1-2 a q_{j}^{*}-C_{a}\right) A_{j} \mu_{j}^{t_{k}}<\left(1-2 a q_{k}^{*}-C_{a}\right) A_{k} \mu_{k}^{t_{k}}$, then the attacker obtains more utility by adding $p_{i}^{*}$ to $p_{k}^{*}$ and setting $p_{i}^{*}$ equal to 0 . Similarly, noticing the source's total utility function $U_{S}^{t_{k}}(P, Q)$, it holds that

$$
0 \leq A_{i} \mu_{i}^{t_{k}}\left[p_{i}^{*}\left(2 a+b C_{f}\right)-\left(b C_{f}+C_{m}\right)\right]=A_{j} \mu_{j}^{t_{k}}\left[p_{j}^{*}\left(2 a+b C_{f}\right)-\left(b C_{f}+C_{m}\right)\right]
$$




$$
\begin{gathered}
\geq A_{k} \mu_{k}^{t_{k}}\left[p_{k}^{*}\left(2 a+b C_{f}\right)-\left(b C_{f}+C_{m}\right)\right] \\
\quad \forall i, j, k \in \mathcal{R}, q_{i}^{*}, q_{j}^{*}>0, q_{k}^{*}=0 .
\end{gathered}
$$

To find the Nash equilibrium $\left(P^{*}, Q^{*}\right)$ of the proposed dynamic Bayesian game theoretic approach, we need to reclaim that $A_{i}>A_{j}$, if $i>j$ and $\sum_{i}^{|\mathcal{R}|} p_{i}^{*}=\sum_{i}^{|\mathcal{R}|} q_{i}^{*}=$ 1.

From

$$
\begin{aligned}
& \left(1-2 a q_{i}^{*}-C_{a}\right) A_{i} \mu_{i}^{t_{k}}=\left(1-2 a q_{j}^{*}-C_{a}\right) A_{j} \mu_{j}^{t_{k}}, \\
& A_{i} \mu_{i}^{t_{k}}\left[p_{i}^{*}\left(2 a+b C_{f}\right)-\left(b C_{f}+C_{m}\right)\right]=A_{j} \mu_{j}^{t_{k}}\left[p_{j}^{*}\left(2 a+b C_{f}\right)-\left(b C_{f}+C_{m}\right)\right],
\end{aligned}
$$

we have

$$
\begin{aligned}
p_{i}^{*} & \left.=\frac{b C_{f}+C_{m}}{\mu_{i}^{t_{k}}\left(2 a+b C_{f}\right)}+\frac{A_{j}\left[p_{j} \mu_{j}^{t_{k}}\left(2 a+b C_{f}\right)-\left(b C_{f}+C_{m}\right)\right]}{A_{i} \mu_{i}^{t_{k}}\left(2 a+b C_{f}\right.}\right) \\
q_{i}^{*} & =\frac{1}{2 a}\left[1+C_{a}-\frac{A_{j} \mu_{j}^{t_{k}}\left(1-2 a q_{j}+C_{a}\right)}{A_{i} \mu_{i}^{t_{k}}}\right]
\end{aligned}
$$

For the proposed dynamic Bayesian game theoretic approach, Nash equilibrium $\left(P^{*}, Q^{*}\right)$ at stage $t_{k}$ is given as follows:

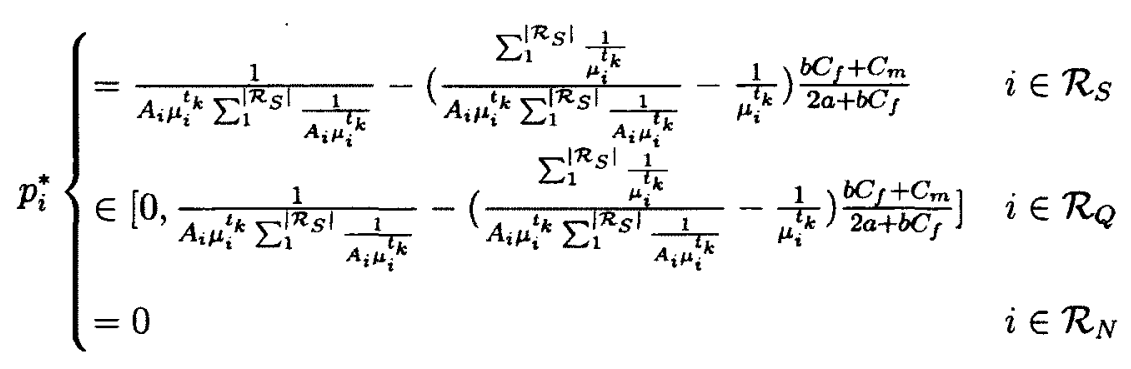




$$
q_{i}^{*}= \begin{cases}\frac{1}{2 a}\left(1-C_{a}-\frac{\left|\mathcal{R}_{S}\right|\left(1-C_{a}\right)-2 a}{A_{i} \mu_{i}^{t_{k}} \sum_{1}^{\mid \mathcal{R} S} \frac{1}{A_{i} \mu_{i}^{t_{k}}}}\right) & i \in \mathcal{R}_{S} \\ 0 & \text { otherwise }\end{cases}
$$

where

$$
\sum_{i \in \mathcal{R}} p_{i}^{*}=\sum_{i \in \mathcal{R}} q_{i}^{*}=1
$$

Nash equilibrium $\left(P^{*}, Q^{*}\right)$ of the proposed static game theoretic approach is the special case of the Nash equilibrium of the proposed dynamic Bayesian game theoretic approach by setting $\mu_{i}$ equal to 1 , which assumes all candidate relay nodes are completely malicious.

\subsection{System Performance Analysis}

In our model, the system security requirement is defined as the maximum percentage of packets forwarded to the destination through the selected relay that are compromised by the attacker if the attacker's attacking target selection coincides with the source's relay selection. Denote the utility brought by a successful attack on targeted relay $R_{i}$ as $u_{A}\left(p_{i}, q_{i}\right)$. We assume that the attacker prefers selecting relay $R_{i}$ with the attacking probability $p_{i}^{*}$ that maximizes $u_{A}\left(p_{i}, q_{i}\right)$ as its attacking target; attacker's attacking target selection may coincide with source's relay selection. If the attacker's selection coincides with the relay selection of the source, then both identity-authentication and packet-integration checking processes are needed to guarantee a secured communication. However, when a decision on relay selection is made, the source could not make sure which relay is the target of the attacker except for 
a probability of being attacked; but the source could detect the attack initiated by the attacker on relays. Therefore, with the satisfaction of the system security requirement, the source would not necessarily authenticate all packets, according to the possibility that packets forwarded by the selected relay are not compromised by the attacker because the source's relay selection is different from the attacker's attacking target selection. Since not all the packets sent by the source are needed to be authenticated, compared with the stringent authentication relay selection method [1], which authenticates all transmitted packets, the proposed game theoretic approach provides a quantitative approach to calculate the authentication probability based on the attacker's attacking probabilities on relays and system security requirement; and to avoid the unnecessary consumption of system resources.

Denote the probability of message authentication as $p_{a}$. To satisfy system security requirement $p_{s}$, we have $0 \leq\left(1-p_{a}\right) \cdot p_{i}^{*} \leq p_{s}$ by selecting relay $R_{i}$ as relay with probability $p_{i}^{*}$ being attacked by the attacker.

\subsubsection{Outage Probability and Capacity}

In the proposed game theoretic approach, denote $I_{i}$ as maximum value between the mutual information of direct Communication $I_{D C}$ and the minimal value between $I_{S R_{i}}$, the mutual information between the source and the selected relay $R_{i}$, and $I_{M R C}$, the mutual information sum of source-destination and relay $R_{i}$-destination [44]. We define SNR as the average signal-to-noise ratio from the source node to the destination node [7]. $I_{D C}$ is given by

$$
I_{D C}=\log _{2}\left(1+\left|h_{S D}\right|^{2} \mathrm{SNR}\right)
$$


and $I_{S R_{i}}$ is given by

$$
I_{S R_{i}}=\frac{1}{2} \log _{2}\left(1+\left|h_{S R_{i}}\right|^{2} \mathrm{SNR}\right)
$$

where $\left|h_{S R_{i}}\right|$ is the channel between the source and relay $R_{i}$. Given the half-duplex constraint in cooperative wireless communication networks which means a relay could not transmit and receive signal simultaneously, the factor $\frac{1}{2}$ mirrors the two time slots for relaying. $I_{M R C}$ is given by

$$
I_{M R C}=\frac{1}{2} \log _{2}\left(1+\left(\left|h_{S D}\right|^{2}+\left|h_{R_{i} D}\right|^{2}\right) \mathrm{SNR}\right)
$$

where $\left|h_{S D}\right|$ is the channel between the source and the destination; $\left|h_{R_{i} D}\right|$ is the channel between the selected relay $R_{i}$ and the destination.

Suppose the data transmission rate of the cooperative wireless communication between the source and the destination is $r$. Outage probability $P_{\text {out }}^{I_{i}}$ is defined as the probability that the mutual information $I_{i}$ between the source and the destination through relay $R_{i}$ is lower than the transmission data rate $r$, i.e., $P_{\text {out }}^{I_{i}}=P\left\{I_{i}<r\right\}$, which characterizes the probability of transmission data loss.

In the case of the proposed game theoretic approach, the outage probability is defined as below:

$$
P_{\text {out }}^{I_{i}}=P\left\{\max \left\{I_{D C}, \min \left\{I_{S R_{i}}, I_{M R C}\right\}\right\}<r\right\}
$$

from which we can obtain,

$$
P_{o u t}^{I_{i}}=1-v+\frac{\omega^{\left(d_{S R_{i}}^{\alpha}+d_{R_{i} D}^{\alpha}\right)}\left(v^{\left(1-d_{R_{i} D}\right)}-1\right)}{1-d_{R_{i} D}^{\alpha}}
$$

where $\omega$ equals to $\exp \left(2 \ln v-(\ln v)^{2} \gamma\right)$ and $v$ equals to $\exp \left(-\frac{2^{r}-1}{\gamma}\right) \cdot d_{S R_{\mathrm{i}}}$ denotes the 
distance between the source and selected relay $R_{i}, d_{R_{i} D}$ denotes the distance between selected relay $R_{i}$ and the destination, and $\gamma$ denotes the average transmitted SNR between any nodes.

The outage capacity $C_{\epsilon}^{I}$ is defined as the largest data transmission rate $r$ that can be supported if the outages are allowed to occur with a certain probability $\epsilon$, which is the probability that the transmission cannot be decoded with negligible error probability. Solving $P_{\text {out }}^{I_{i}}=\epsilon$, we have $v_{\epsilon}$. Thus, we have,

$$
C_{\epsilon}^{I}=\log _{2}\left(1+\gamma \ln \left(\frac{1}{v_{\epsilon}, \gamma}\right)\right)
$$

Outage capacity is used instead of Shannon capacity in slow fading channel since the slow fading channel is different from the additive white Gaussian noise channel as delay constraints on the order of channel coherence time.

\subsubsection{Bit Error Rate}

Bit Error Rate (BER) is the percentage of bits that have errors relative to the total number of bits sent in a transmission [45]. The end-to-end BER, is given by

$$
P_{e}^{I_{i}}=P_{o u t}^{S R_{i}} \cdot P_{e}^{D C}+\left(1-P_{\text {out }}^{S R_{i}}\right) \cdot P_{e}^{d i v, i},
$$

where $P_{\text {out }}^{S R_{i}}$ is the outage probability of the link from the source to the selected relay $R_{i}[46], P_{e}^{D C}$ is the probability of error in direct communication form source to destination over Rayleigh channel and $P_{e}^{d i v, i}$ is the probability that an error occurs in combined transmission from the source to the destination through the selected relay $R_{i} . P_{\text {out }}^{S R_{i}}$ is given as below:

$$
P_{\text {out }}^{S R_{i}}=1-\exp \left(-\left(\frac{2^{2 r}-1}{\overline{\gamma_{S R_{i}}}}\right)\right) .
$$


$P_{e}^{D C}$ is given by

$$
P_{e}^{D C}=\frac{1}{2}\left(1-\sqrt{\frac{\overline{\gamma_{S D}}}{1+\overline{\gamma_{S D}}}}\right)
$$

$P_{e}^{d i v, i}$ is given as follows:

$$
P_{e}^{d i v, i}=\frac{1}{2}\left[1+\frac{1}{\overline{\gamma_{R_{i} D}}-\overline{\gamma_{D C}}}\left(\frac{\overline{\gamma_{D C}}}{\sqrt{1+\frac{1}{\gamma_{D C}}}}-\frac{\overline{\gamma_{R_{i} D}}}{\sqrt{1+\frac{1}{\overline{\gamma_{R_{i} D}}}}}\right)\right]
$$

where $\overline{\gamma_{D C}}$ denotes the SNR between the source and the destination and $\overline{\gamma_{R_{i} D}}$ denotes the SNR between the selected relay $R_{i}$ and the destination.

\subsubsection{System Throughput}

We derive the throughput for partial authentication process with ALPHA-M protocol [21], and modify it to cover both direct communication and source-relaydestination communication. Furthermore, we formulate the throughput equations for both Selective Repeat ARQ [47] and Go-Back-N ARQ [48] retransmission schemes by taking the error rate into consideration.

The payload for packets with authentication is given as follow:

$$
S_{\text {paylaod }}=n \cdot p_{a} \cdot\left(S_{\text {packet }}-S_{h}\left(\left\lceil\log _{2}(n)\right\rceil+1\right)\right)
$$

where $S_{\text {payload }}$ is the amount of payload that can be transmitted with a single presignature, $n$ is the number of data blocks at the bottom of Merkle tree, $S_{\text {packet }}$ is the size of packet, and $S_{h}$ is the hash output [21].

The payload for packets without authentication is

$$
S_{\text {payload }}^{\prime}=n \cdot\left(1-p_{a}\right) \cdot\left(S_{\text {packet }}-S_{h}\right)
$$


Table 7: Time parameters in $T_{1}$

\begin{tabular}{|c|c|c|c|c|}
\hline & $t_{\text {prop } 1}$ & $t_{f 1}$ & $t_{\text {proc1 } 1}$ & $t_{a c k 1}$ \\
\hline$T_{1}^{D C}$ & $2\left(\frac{d_{S D}}{c}\right)$ & 1 & 3 & 1 \\
\hline$T_{1}^{S R D}$ & $2\left(\frac{d_{S R_{i}}}{c}\right)+2\left(\frac{d_{R_{i} D}}{c}\right)$ & 2 & 5 & 2 \\
\hline
\end{tabular}

Table 8: Time parameters in $T_{2}$

\begin{tabular}{|c|c|c|c|c|}
\hline & $t_{\text {prop } 2}$ & $t_{f 2}$ & $t_{\text {proc } 2}$ & $t_{\text {ack2 }}$ \\
\hline$T_{2}^{D C}$ & $2\left(\frac{d_{S D}}{c}\right)$ & $n$ & 1 & 1 \\
\hline$T_{2}^{S R D}$ & $2\left(\frac{d_{S R_{i}}}{c}\right)+2\left(\frac{d_{R_{i} D}}{c}\right)$ & $n+1$ & 3 & 2 \\
\hline
\end{tabular}

Generally, throughput is defined as the payload divided by the total time used for processing and transmitting the payload. In our case, the total time spent on payload processing and transmitting consists of two parts: $T_{1}$, the time for the initial pre-signature process between the source and the destination; and $T_{2}$, the time for the actual authenticated and non-authenticated message transmission and delivery. Then, the general throughput $\mathrm{T}$ could be defined as:

$$
\mathrm{T}=\frac{S_{\text {payload }}+S_{\text {payload }}^{\prime}}{T_{1}+T_{2}}
$$

The values for the time parameters in $T_{1}$ and $T_{2}$ vary according to two communication paths, direct communication and source-relay-destination, which are presented in Table 7 and Table 8.

The message sequence charts that show the transmission of message from the source to the destination and acknowledgment between the destination and the source with and without the use of relay are shown in Figure 7.

The parameters presented in Table 7 and Table 8 are explained as follows:

- $t_{\text {prop } 1}$ is the propagation time for the $S_{1}$ packet from the source to the destination or the propagation time for the $A_{1}$ packet sent from the destination to the source. 
In the case of direct communication, $t_{\text {prop } 1}$ is given by $\frac{d_{S D}}{c}$, where $d_{S D}$ is the distance between the source and the destination and $c$ is the speed of light. In the case of source-relay-destination, this consists of the time for the $S_{1}$ packet sent from the source to the selected relay $R_{i}$ and from the selected relay $R_{i}$ to the destination; or for the $A_{1}$ packet sent from the destination to the selected relay $R_{i}$ and from the selected relay $R_{i}$ to the source, which is given by the sum of $\frac{d_{S R_{i}}}{c}$ and $\frac{d_{R_{i} D}}{c}$.

- $t_{\text {prop } 2}$ is the propagation time for the $S_{2}$ packet from the source to the destination; or for the $A_{2}$ packet from the destination to the source. In the case of direct communication, this is given by $\frac{d_{S D}}{c}$. In case of source-relay-destination, this consists of the propagation time for the $S_{2}$ packet from the source to the selected relay $R_{i}$ and from the selected relay $R_{i}$ to the destination or for the $A_{2}$ packet from the destination to the selected relay $R_{i}$ and from the selected relay $R_{i}$ to the source, which is given by the sum of $\frac{d_{S R_{i}}}{c}$ and $\frac{d_{R_{i} D}}{c}$.

- $t_{f 1}$ is the packet transmission time for the $S_{1}$ packet, which is given by $\frac{u_{f 1}}{r} . u_{f 1}$ is the number of bits in the $S_{1}$ packet and $r$ is the data transmission rate.

- $t_{f 2}$ is the packet transmission time for the $S_{2}$ packet, which is given by $\frac{u_{f 2}}{r} \cdot u_{f 2}$ is the number of bits in the $S_{2}$ packet and $r$ is the data transmission rate.

- $t_{a c k 1}$ is the packet transmission time for the. $A_{1}$ packet, which is given by $\frac{u_{a c k 1}}{r}$. $u_{a c k 1}$ is the number of bits in the $A_{1}$ packet and $r$ is the data transmission rate.

- $t_{a c k 2}$ is the packet transmission time for the $A_{2}$ packet, which is given by $\frac{u_{a c k 2}}{\tau}$. $u_{a c k 2}$ is the number of bits in the $A_{2}$ packet and $r$ is the data transmission rate.

- $t_{\text {proc1 }}$ is the processing time at the source and the destination for $S_{1}$ and $A_{1}$ packets in direct communication, which includes the Merkle tree generating 
time for $S_{1}$ packet at the source and the acknowledgment Merkle tree for $A_{1}$ packet at the destination along with processing at the selected relay $R_{i}$ in sourcerelay-destination.

- $t_{p r o c 2}$ is the processing time at the source and the destination for $S_{2}$ and $A_{2}$ packets in direct communication, along with processing time at the selected relay $R_{i}$ in source-relay-destination.

Wireless channels have high error rates due to multi-path fading which characterizes mobile radio channels. However, many networks require that the error rates should be significantly small. In addition to the poor channel quality, the design of wireless communication systems is complicated by the rapidly changing quality of the radio channel [49]. To increase the apparent quality of a communication channel, two distinct approaches are used:

- Forward Error Correction (FEC) which employs error correcting codes to combat bit errors which are due to channel imperfections by adding redundancy, such as henceforth parity bits, to information packets before they are transmitted. This redundancy is used by the receiver to detect and correct errors that are introduced in the transmission process.

- Automatic Repeat Request (ARQ) wherein only error detection capability is provided and no attempt to correct any packets received in error is made. Packets received in error are retransmitted by the sender.

In the throughput analysis, ARQ retransmission is incorporated; and the following is a brief review of three typical ARQ retransmission schemes [50].

- Stop and Wait (SW) ARQ: When using the SW ARQ scheme, the sender transmits a packet only when all previously transmitted packets have been successfully acknowledged by the receiver. Hence, when using SW ARQ scheme, the 
sender after transmitting a packet waits for its acknowledgment. Once its acknowledgment has been received, the next packet is transmitted. However, if an acknowledgment does not arrive until a timeout timer expires, the packet is retransmitted by the sender. Therefore, in SW ARQ there is never more than a single packet that is unacknowledged at any given instant of time. Since the sender does not use the available channel during time intervals it waits for an acknowledgment, the maximum data transfer rate that can be supported is limited. This limits cases where the SW ARQ protocol can be employed. Huge buffer is needed to buffer un-acknowledged packets.

- Selective Repeat (SR) ARQ: Unlike SW ARQ, when using SR ARQ, packets are transmitted continuously by the sender. As before, the receiver acknowledges each successfully received packet by transmitting an ACK bearing the sequence number of the packet being acknowledged. If an acknowledgment is not received for a packet before the expiration of the timeout, the packet is retransmitted. Once a packet has been retransmitted, the sender resumes transmission of packets from where it is left off, i.e., if $a$ is the packet with the largest sequence number that has been transmitted, packet with sequence number $a+1$ is transmitted next. Here we assume that no other timers have expired in the meantime. Since the SR ARQ protocol is employed, packets are continuously being transmitted, the inefficiency associated with SW ARQ is eliminated. Observe that when SR ARQ is employed, packets can be accepted out of sequence. Hence, packets received out of sequence have to be buffered and sequenced before they can be delivered.

- Go-Back-N (GBN) ARQ: When GBN ARQ is employed, packets are transmitted continuously as in SR ARQ. However, the receiver accepts packets only in the order in which they were transmitted. Packets received out of sequence are 
discarded and not acknowledged. Since the receiver accepts packets only insequence, after a timeout, the sender retransmits the packet that timed out and all packets with sequence numbers that follow the one that was retransmitted. Hence, each time a timeout occurs, all packets that are yet to be acknowledged are retransmitted. It is important to observe that GBN ARQ attempts to combine the desirable features of SR and SW ARQ, i.e., packets are transmitted continuously, as in SR ARQ, without the need to buffer out of sequence packets and there is no re-sequencing overhead.

To incorporate the error control schemes into our throughput equation, we expand the general throughput equation by including the error rate. Define the packet error rate $P_{\mathrm{c}}$ as the probability that the received packet with the length of $S_{\text {packet }}$ bits contains no error as $P_{c}=\left(1-P_{e}^{I_{i}}\right)^{S_{\text {packet }}}$. Let $\mathrm{T}_{S R}$ denote the modified throughput with SR ARQ, which is given as below,

$$
\mathrm{T}_{S R}=\frac{\left(S_{\text {payload }}+S_{\text {payload }}^{\prime}\right) \cdot P_{c}}{T_{1}+T_{2}}
$$

Concerning the GBN ARQ, the throughput equation is further modified to allow the retransmission of an error frame along with all frames that have been transmitted until the time a negative acknowledgment is received from the destination. Thus, the modified throughput with GBN ARQ, denoted by $\mathrm{T}_{G B N}$, is given as,

$$
\mathrm{T}_{G B N}=\frac{\left(S_{\text {payload }}+S_{\text {payload }}^{\prime}\right) \cdot P_{c}}{T_{1}+T_{2}\left[P_{c}+\left(1-P_{c}\right) W_{s}\right]}
$$

where $W_{s}$ is the window size which is calculated by dividing the product of the data rate of the transmission channel and the reaction time by the packet size. 


\subsubsection{Optimizing Number of Message}

Besides strategically selecting relay, the source also needs to determine the optimal number of messages once its relay is selected. For various packet sizes $S_{\text {packet }}$ and authentication probability $p_{a}$, the optimal value of the number of messages $n$ that results in the highest throughput is denoted as $n^{*}$. The optimal number of messages for selected relay $R_{i}$ is driven from

$$
n^{*}=\arg \max _{n} \mathrm{~T}\left(R_{i}, S_{\text {packet }}, n, p_{a}\right)
$$

where $n \in\{1,2, \ldots\}$ for the selected relay $R_{i}$.

In the next chapter, simulation results and discussion are presented to show the effectiveness of the proposed game theoretic approach for security and QoS co-design in cooperative wireless communication networks. The correctness of system performance analysis is also presented in the next chapter. 

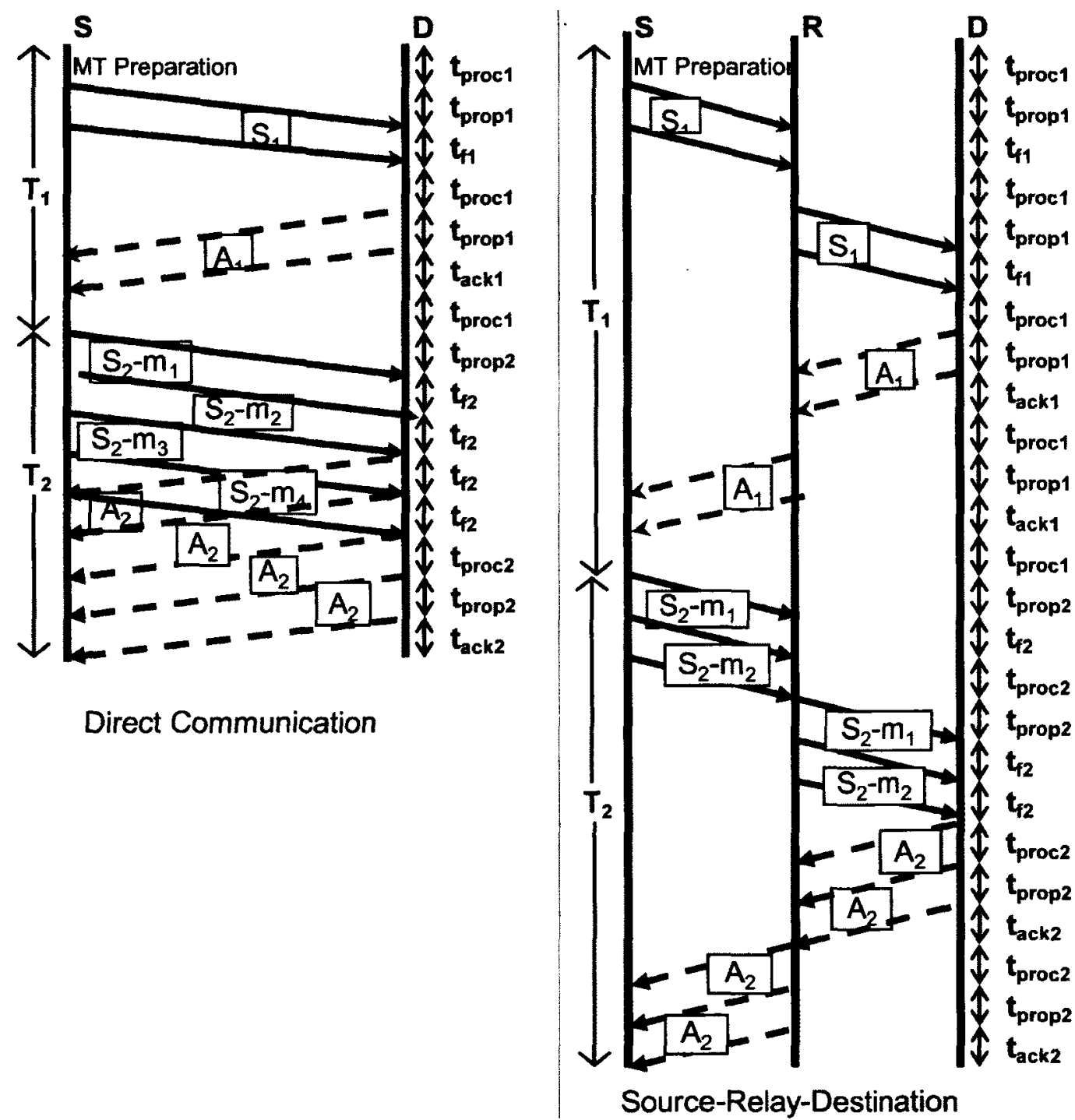

Figure 7: Message sequence charts in direct communication and source-relaydestination communication 


\section{Chapter 4}

\section{Simulation Results and Discussion}

In this chapter, we evaluate the performance of the proposed game theoretic approach for security and QoS co-design in cooperative wireless communication networks through extensive simulations using MATLAB. All simulations are executed on a laptop featured with Windows 7, Intel Core Duo $2.1 \mathrm{GHz}$ CPU, 2 GB memory and MATLAB R2010b. As illustrated by Figure 8, we set up a network topology with the source and the destination located 1000 meters apart in two separate corners, and four relays randomly located between the source and the destination in an area of $1000 \times 300 \mathrm{~m}^{2}$. We set the transmission data rate equal to $1 \mathrm{Mbps}$, path loss exponent equal to 3.5 and fixed outage probability equal to 0.01 .

\subsection{Simulation Scenarios}

In this section, we perform a numerical study on two typical networks to validate our analytical results in attacking target selection and relay selection.

Similar to [37], first of all, we consider a network with emphasis on system security, e.g., military network, where there are tight confidential requirements. In this network, the security asset weights heavier than the information asset and the combined asset is much higher than attack monitoring cost, e.g., $\alpha_{I}<\alpha_{S}$ and $C_{a}, C_{m}, C_{f} \ll 1$. 


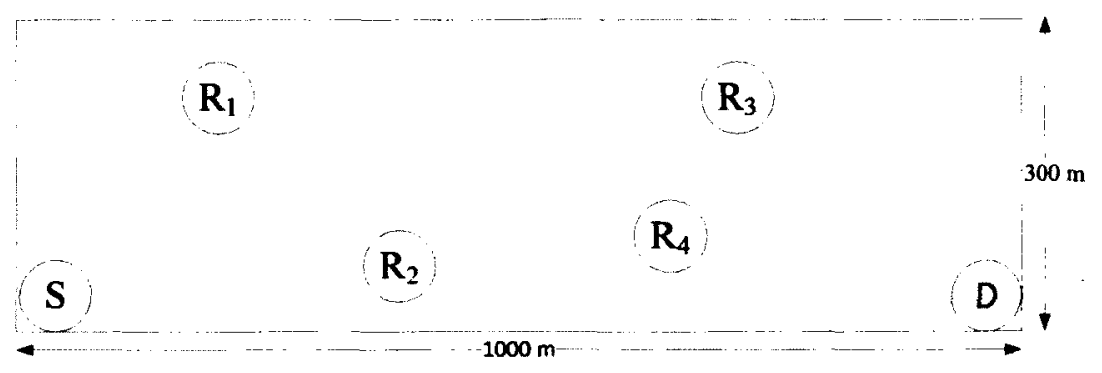

Figure 8: Simulation topology

We set $C_{a}=C_{m}=0.01$ and $C_{f}=0.01$. Terminals in military network usually own high-performance attack monitoring equipments and powerful processing capability, thus we set $a=0.9$ and $b=0.05$.

Secondly, a network with loose emphasis on system security is considered, e.g., commercial WLAN. In this network, the information asset weights heavier than the security asset and the related attacking and attack monitoring cost is relatively high, i.e., $\alpha_{I}>\alpha_{S}$; and we set $C_{a}=C_{m}=0.1$ and $C_{f}=0.3$. The terminals in commercial network are not as efficient as those in military network, thus we set $a=0.6$ and $b=0.2$.

In both networks, there are four relays with normalized information and security assets: $A_{i}=(5-i) \cdot 0.25, i=\{1,2,3,4\}$. Table 9 and Table 10 show the $\operatorname{NE}\left(P^{*}, Q^{*}\right)$ of the proposed static game theoretic approach obtained by using analytical results in Chapter 3. As shown in Table 9 and Table 10, both the attacker and the source focus only on the relays in the sensible target set, which bring them more utility.

The attacker would choose the relay that brings maximum attacking utility as its attacking target. According to the obtained Nash equilibrium, the attacker in military network is prone to select Relay 3 as its attacking target. However, in real network, attacking target is selected randomly by the attacker. To simulate the randomness of 
Table 9: Nash equilibrium and players' utility in the military network

\begin{tabular}{|c|}
\hline Nash equilibrium \\
\hline$p_{1}^{*}=0.23256, q_{1}^{*}=0.4$ \\
$p_{2}^{*}=0.30814, q_{2}^{*}=0.35$ \\
$p_{3}^{*}=0.4593, q_{3}^{*}=0.25$ \\
$p_{4}^{*}=0, q_{4}^{*}=0$ \\
\hline Players' Utility \\
\hline$u_{A}\left(p_{1}^{*}, q_{1}^{*}\right)=0.062792, u_{D}\left(p_{1}^{*}, q_{1}^{*}\right)=-0.069271$ \\
$u_{A}\left(p_{2}^{*}, q_{2}^{*}\right)=0.083198, u_{D}\left(p_{2}^{*}, q_{2}^{*}\right)=-0.088225$ \\
$u_{A}\left(p_{3}^{*}, q_{1}^{*}\right)=0.12401, u_{D}\left(p_{1}^{*}, q_{1}^{*}\right)=-0.12759$ \\
$u_{A}\left(p_{4}^{*}, q_{4}^{*}\right)=0, u_{D}\left(p_{4}^{*}, q_{4}^{*}\right)=0$ \\
\hline
\end{tabular}

Table 10: Nash equilibrium and players' utility in the commercial network

\begin{tabular}{|c|}
\hline Nash equilibrium \\
\hline$p_{1}^{*}=0.26984, q_{1}^{*}=0.46154$ \\
$p_{2}^{*}=0.31746, q_{2}^{*}=0.36583$ \\
$p_{3}^{*}=0.4127, q_{3}^{*}=0.17308$ \\
$p_{4}^{*}=0, q_{4}^{*}=0$ \\
\hline Players' Utility \\
\hline$u_{A}\left(p_{1}^{*}, q_{1}^{*}\right)=0.093407, u_{D}\left(p_{1}^{*}, q_{1}^{*}\right)=-0.18676$ \\
$u_{A}\left(p_{2}^{*}, q_{2}^{*}\right)=0.10989, u_{D}\left(p_{2}^{*}, q_{2}^{*}\right)=-0.17233$ \\
$u_{A}\left(p_{3}^{*}, q_{3}^{*}\right)=0.14286, u_{D}\left(p_{3}^{*}, q_{3}^{*}\right)=-0.1752$ \\
$u_{A}\left(p_{4}^{*}, q_{4}^{*}\right)=0, u_{D}\left(p_{4}^{*}, q_{4}^{*}\right)=0$ \\
\hline
\end{tabular}


attacker's selection on attacking target, we generate random numbers $r^{\prime}$ that satisfy 0-1 uniform distribution and set following attacking target selection standard, e.g., if $(i-1) * 0.25 \leq r^{\prime}<i * 0.25, i=\{1,2,3,4\}$, Relay $i$ is selected as attacking target.

\subsection{Optimal Number of Messages}

Figure 9 shows the throughput vs. the number of messages. Figure 9 indicates that the number of messages, exerts a dramatic effect on system throughput. Initially, the system throughput starts to increase with the increment of the number of messages, but then decreases as the increment of large overhead introduced into the system. The large overhead refer to the payload $B_{C}$, the sibling nodes from the leaves to the root. Subsequently, the system throughput drops to zero. System throughput results that are lower than zero are omitted in Figure 9. Therefore, the number of messages, which provides the highest throughput for given packet size and given authentication probability, should be selected as the optimal number of messages.

\subsection{Effect of Processing Time on the Optimal Number of Messages}

Since there are frequently heterogeneous wireless terminals in cooperative wireless communication networks with various processing capabilities, thus the processing time for security services varies and exerts a significant impact on the optimal number of messages selection. In Figure 10 and Figure 11, we observe that as the increment of the processing time, the optimal $n$ value changes for almost all packet sizes

$S_{\text {packet }}$. Therefore, the optimal $n$ value will be different for each network and has to be recalculated for each specific network to obtain the maximum throughput. 


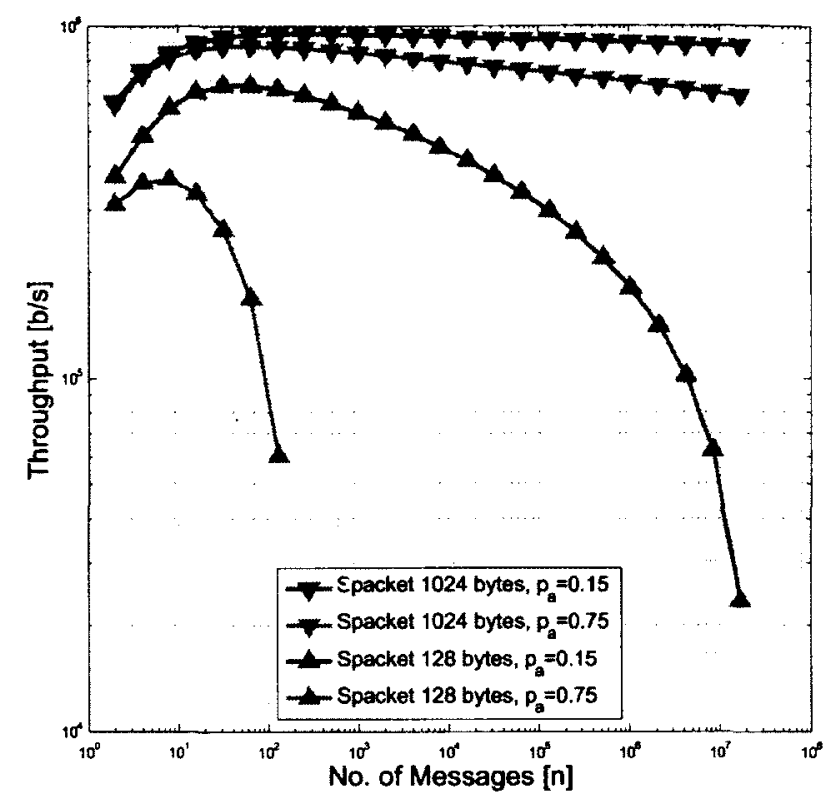

Figure 9: The effect of the number of messages on system throughput

\subsection{Effect of Authentication Probability on Throughput and Compromising Probability}

Since not all the packets sent by the source are authenticated with the satisfaction of system security requirement, values of authentication probability exert impact on the system throughput and compromising probability. Simulations concerning system throughput analysis integrated with Selective Repeat ARQ and Go-Back-N ARQ and compromising probability are conducted.

Figure 12 shows the simulation results of system throughput of military network obtained by adopting Selective Repeat ARQ and Go-Back-N ARQ vs. authentication probability. Simulation results indicate that with the increment of authentication probability, system throughput decreases; at 100 percent authentication probability, the system throughput degrades to that obtained in [1]. This shows that system throughput obtained by applying the proposed game theoretic approach is superior 


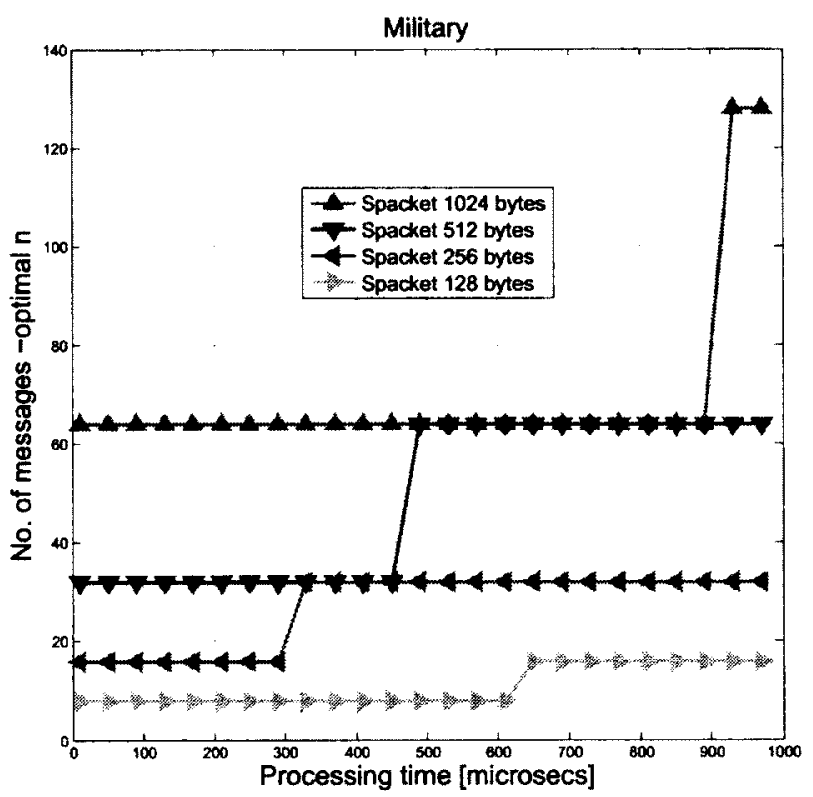

Figure 10: Change in optimal $n$ for different processing time of terminals in military network, $p_{a}=0.75$

to the existing approach that applies stringent authentication protocol. We could also observe that throughput obtained by incorporating Selective Repeat ARQ is better than that obtained by incorporating Go-Back-N ARQ, which is due to the fact that any error happened in transmission process needs the retransmission of all packets within the window in the Go-Back-N ARQ scheme. Similar analysis applies to the simulation results obtained for commercial network as shown by Figure 13.

System compromising probability is 0 while complete authentication scheme is applied. We set the system security requirement as 0.20 , which means there are at most 20 packets in every 100 packets sent by the source tampered by the attacker and could not be used by the destination to recover the original information sent by the source. Figure 14 shows that values of system compromising probability decrease as the authentication probability increases. 


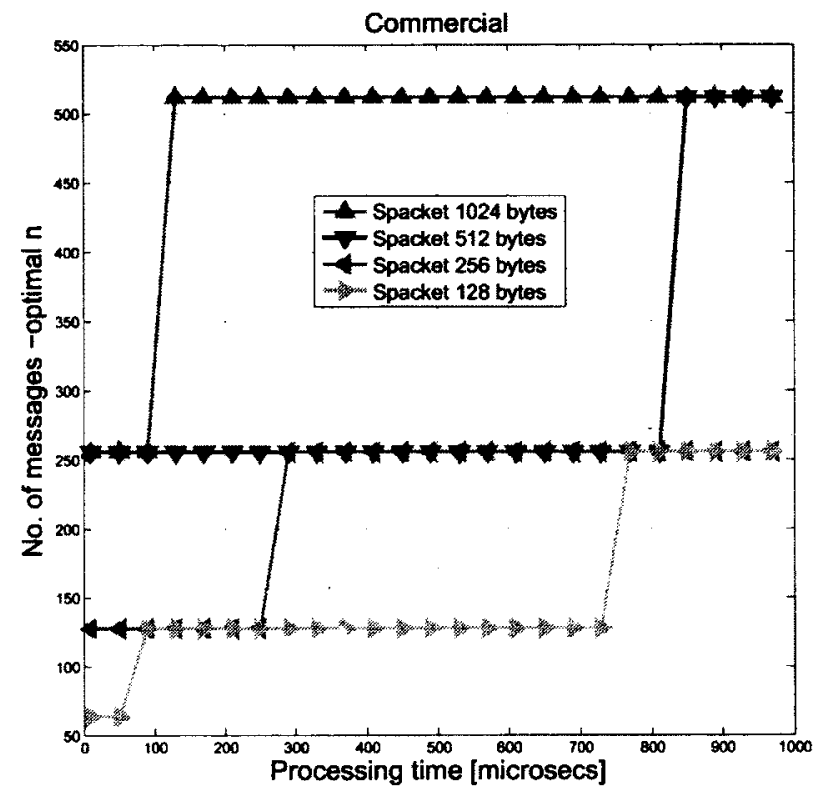

Figure 11: Change in optimal $n$ for different processing time of terminals in commercial network, $p_{a}=0.15$

\subsection{Dynamic Beliefs in the Maliciousness of Relays}

In this section, we discuss dynamic beliefs in the maliciousness of relays according to the attacker's attacking histories on relays and dynamic total utility of the source brought by its dynamic beliefs in the maliciousness of relays. At each stage, the source updates its belief in maliciousness of the selected relay according to its record of attacker's attack on the selected relay. At each stage, if the selected relay by the source is also selected by the attacker as attacking target, packets sent to the destination through the selected relay are considered compromised and could not be used by the destination to recover the original information sent by the source; otherwise, packets sent through the selected relay arrive at the destination without being compromised and could be used by the destination to recover the original information.

Figure 15 shows the simulation results of dynamic change of attacking target of 


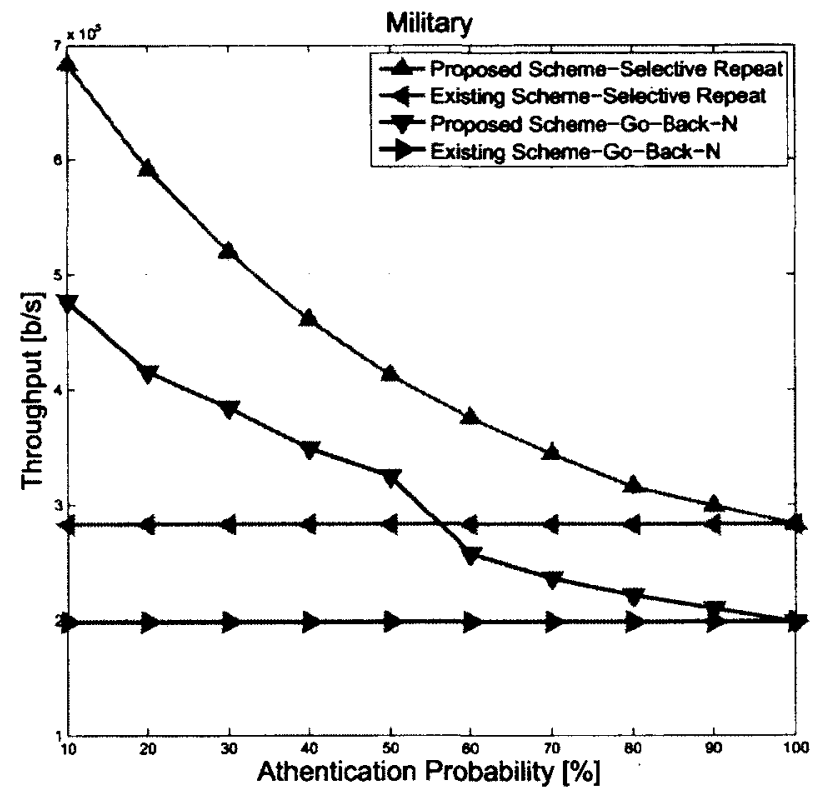

Figure 12: Effect of authentication probability on throughput of military network, $\mathrm{SNR}=30 \mathrm{~dB}$

the attacker and the dynamic change of the selected relay of the source for the first 20 consecutive stages of the proposed dynamic game theoretic approach.

Figure 16, Figure 17, Figure 18 and Figure 19 show the dynamic beliefs change of the source in the maliciousness of Relay 1, Relay 2, Relay 3 and Relay 4, respectively. The source updates its beliefs in the maliciousness of relays according to its record of attacker's attack on relays. At the commence of simulations, the source's beliefs in the maliciousness of all relays are unbiased; in another world, belief in the maliciousness and cooperativeness is fifty-to-fifty. Between every two consecutive stages, the source monitors the attacking target selection of the attacker. If the selected relay by the source is not the attacking target, then the source increases its belief in the cooperativeness of the selected relay; if the selected relay is selected as attacking target, then the source increases its belief in the maliciousness of the selected relay; otherwise, other relays are neither selected as relay by the source nor are selected as attacking 


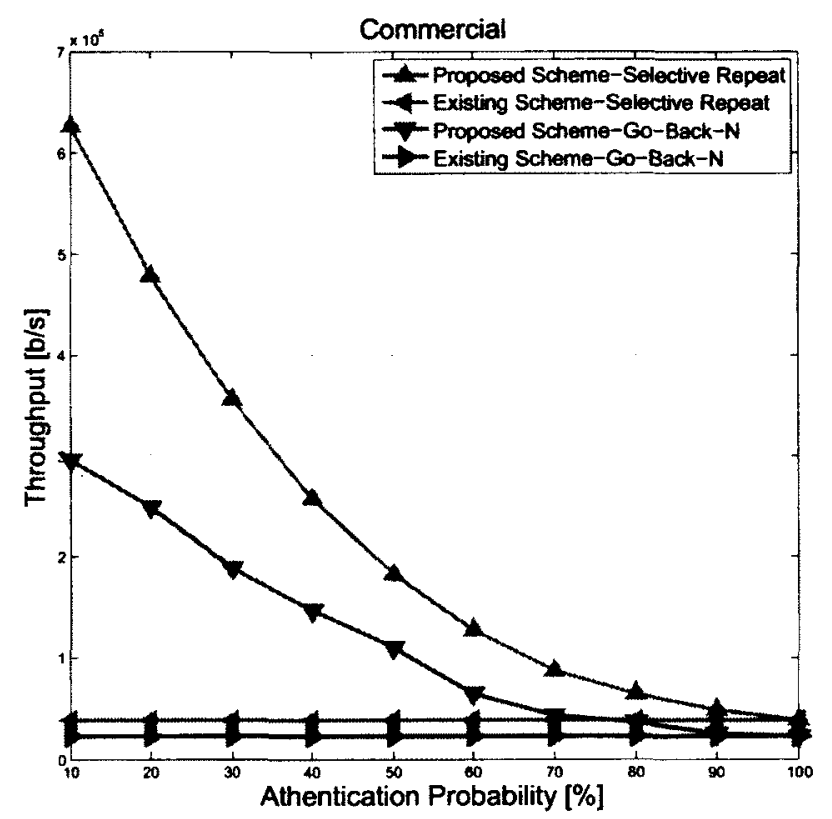

Figure 13: Effect of authentication probability on throughput of commercial network, $\mathrm{SNR}=30 \mathrm{~dB}$

target by the attacker, the source's beliefs in the maliciousness or cooperativeness of other relays stay unchanged.

As shown in Figure 15, at Stage 1, the observed attacking target is Relay 4 and relay selected by the source is Relay 1. At this stage, attacking target does not coincide with the selected relay. Therefore, the source's belief in the maliciousness of Relay 1 decreases, the source's belief in the maliciousness of Relay 4 increases and the source's beliefs in the maliciousness of Relay 2 and Relay 3 stay unchanged. Simulation results in Figure 16 to Figure 19 keep consensus with above analysis.

Figure 20 shows the dynamic change in the total utility of the source in military network and Figure 21 shows the dynamic change in the total utility of the source in commercial network. From these two figures, we could find that the source obtains better total utility compared with that obtained by applying static maliciousness beliefs which assume all the relays are completely malicious all the time instead of 


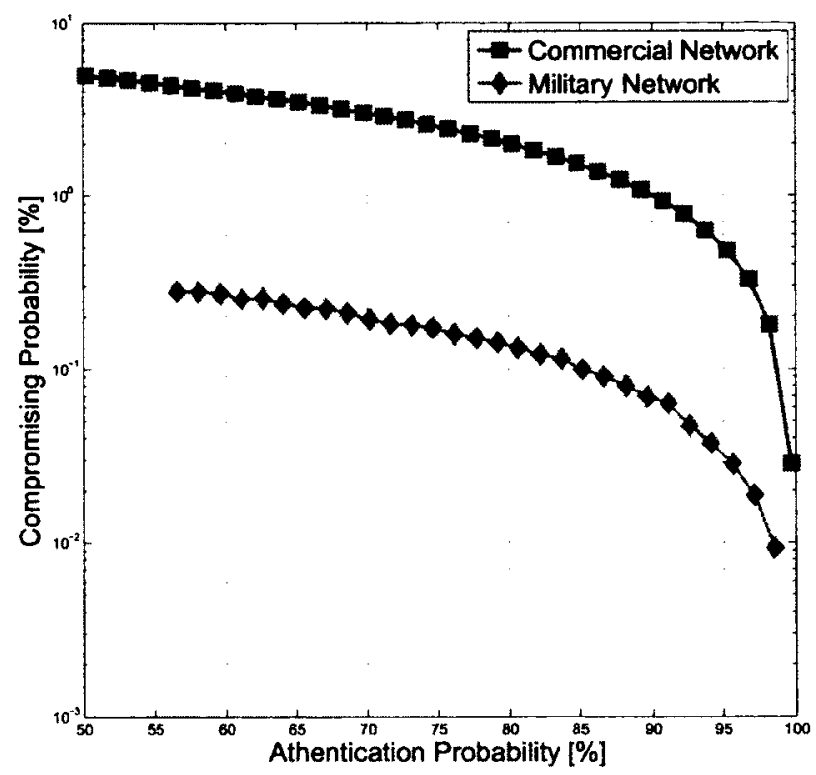

Figure 14: Effect of authentication probability on compromising probability of military and commercial networks

updating beliefs based on the attacker's attacking histories on relays. Figure 22 shows the comparison of the total utility of the source in military and commercial networks in first 20 stages. The source in military network has lower monitoring cost $C_{m}$ and false alarming $\operatorname{cost} C_{f}$, thus when each relay is assigned the same amount of combined information and security assets, the total utility obtained by the source in military network is higher than the total utility obtained by the source in commercial network.

\subsection{Impact of Dynamic Belief Update in the Mali- ciousness of Relays on Throughput and Com- promising Probability}

In this section, we discuss the impact of dynamic belief update in the maliciousness of relays on system throughput and compromising probability of the proposed dynamic 


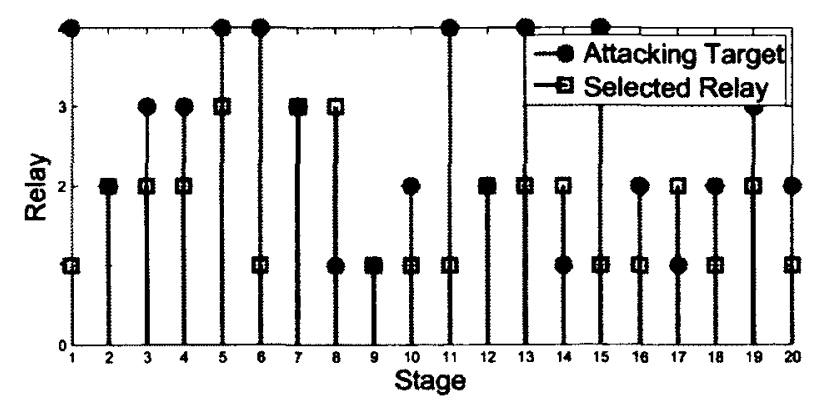

Figure 15: Dynamic attacking target and selected relay

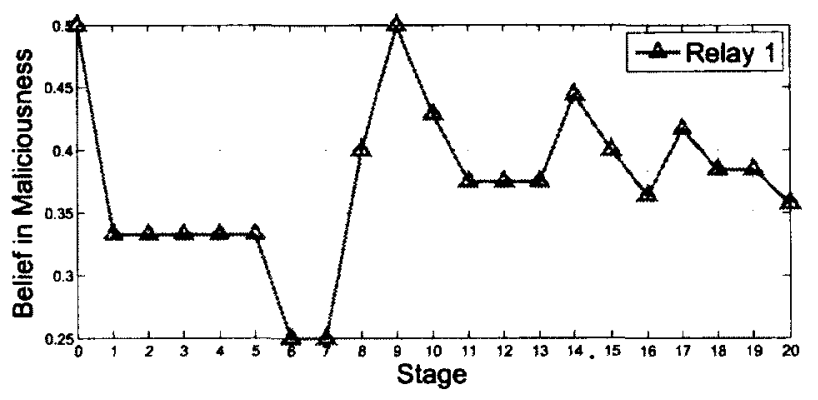

Figure 16: Dynamic belief in the maliciousness of Relay 1

game theoretic approach, which enables the source update its beliefs in the maliciousness of relays based on attacker's attacking histories on selected relays. Numerous simulations are executed to draw reliable results concerning the impact of dynamic beliefs in the maliciousness of relays on throughput and compromising probability.

Figure 23 depicts the simulation results concerning compromising probability of military network obtained by applying static and dynamic game theoretic approaches; similarly Figure 24 depicts the simulation results concerning compromising probability of commercial network obtained by applying static and dynamic game theoretic approaches. As shown by Figure 23, the compromising probability obtained for military network by applying dynamic game theoretic approach is smaller than that obtained by applying static game theoretic approach. Since the source updates it beliefs in the maliciousness of relays according to the attacker's attacking histories on 


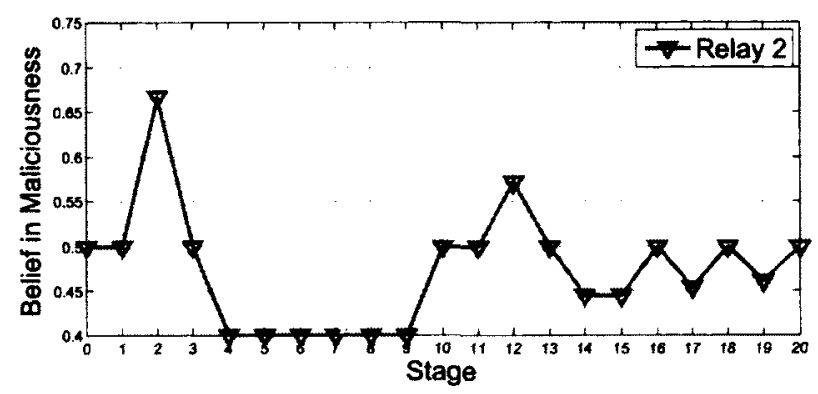

Figure 17: Dynamic belief in the maliciousness of Relay 2

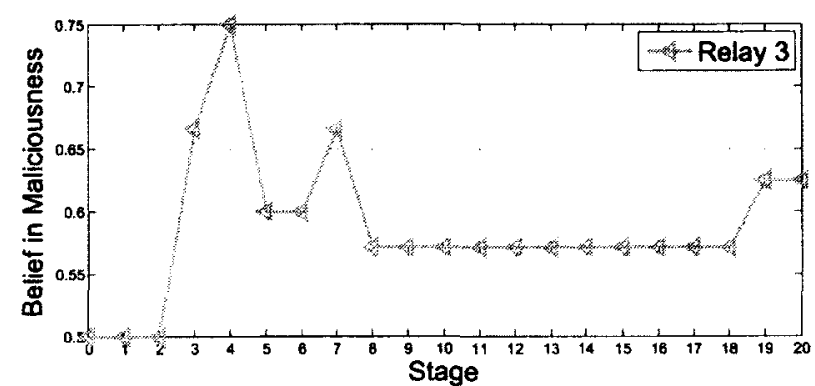

Figure 18: Dynamic belief in the maliciousness of Relay 3

relays, the source thus is prone to select the relay that is more 'trustworthy' and less attacked. Similar analysis is also applicable to the commercial network.

Compromising probability comparison between military and commercial network is shown in Figure 25. As shown by Figure 25, the compromising probability of military network is smaller than the compromising probability of commercial network. Since the security requirement of military network is more stringent than the security requirement of commercial network, the authentication probability of military network is higher than the authentication probability of commercial network.

Figure 26 shows the impact of dynamic belief update in the maliciousness of relays on throughput of military network when SNR equals to $30 \mathrm{~dB}$. As shown by Figure 26, system throughput obtained by applying dynamic game theoretic approach is lower than system throughput obtained by applying static game theoretic approach. In the 


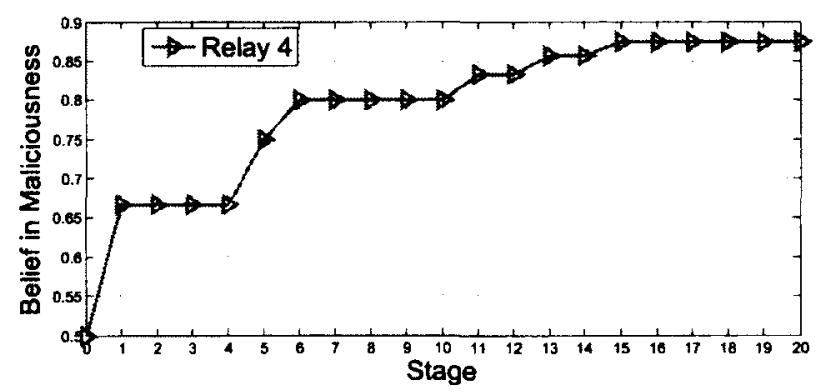

Figure 19: Dynamic belief in the maliciousness of Relay 4

proposed static game theoretic approach, the source selects the relay that brings maximum utility among all candidate relays. The selected relay could be the relay with maximum mutual information. In the proposed dynamic game theoretic approach, the source updates its beliefs in the maliciousness of selected relays according to the attacker's attacking histories on selected relays. If the selected relay is frequently the victim of attacking, the source is not prone to select such frequently attacked relay though it may own maximum mutual information among all candidate relays which directly impacts the system throughput. Since less frequently attacked relay with less mutual information is selected as relay, the system throughput obtained by applying dynamic game theoretic approach is inferior to the system throughput obtained by applying static game theoretic. Similar analysis also applies to the commercial network. As shown by Figure 27, system throughput obtained by applying dynamic game theoretic approach is inferior to system throughput obtained by applying static game theoretic approach.

Figure 28 shows throughput comparison between military and commercial network. As shown by Figure 28, system throughput of military network is superior to the system throughput of commercial network. 


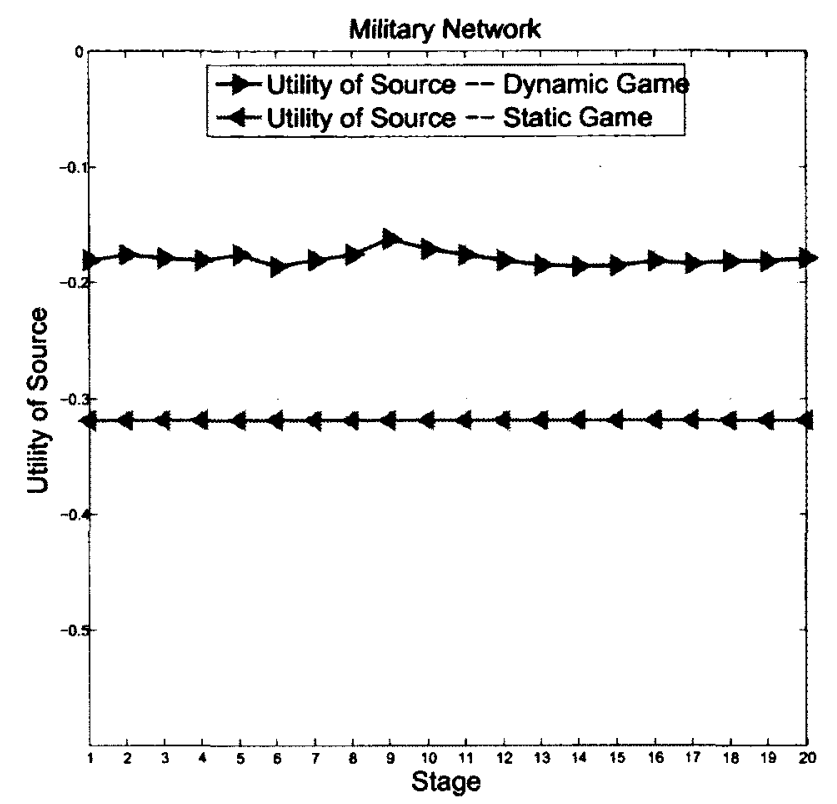

Figure 20: Dynamic total utility of the source in military network

\subsection{Summary}

In this chapter, we present the simulation results to validate our analytical results and effectiveness of the proposed game theoretic approach for security and QoS co-design in cooperative wireless communication networks. We show that optimal number of messages varies with the different packet sizes, authentication probabilities and packet processing time. In another word, optimal number of messages needs to be recalculated for different packet sizes, authentication probability and packet processing time. Simulation results also show that the proposed approach outperforms the existing approach that relies on stringent authentication protocol [1]. The improvement in system throughput is due to the fact that not all the packets sent by the source are authenticated with the satisfaction of system security requirement. Dynamic Bayesian game theoretic approach enables the source to update its beliefs in the maliciousness of relays based on the attacking histories of the attacker. Thus system owns better 


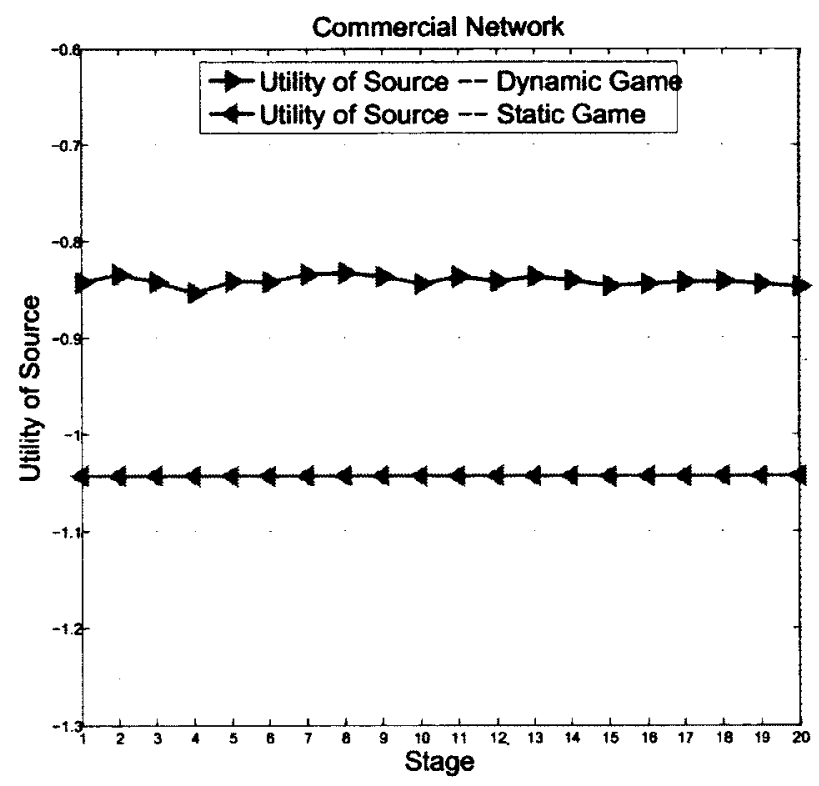

Figure 21: Dynamic total utility of the source in commercial network

compromising probability with slightly inferior system throughput by applying $\mathrm{dy}$ namic game theoretic approach compared with the system performance obtained by applying static game theoretic approach.

The simulation results illustrate the effectiveness of the proposed game theoretic approach, which tries to reach the trade-off between system throughput and system security requirement with acceptable compromising probability. Conclusions and future work are presented in the next chapter. 


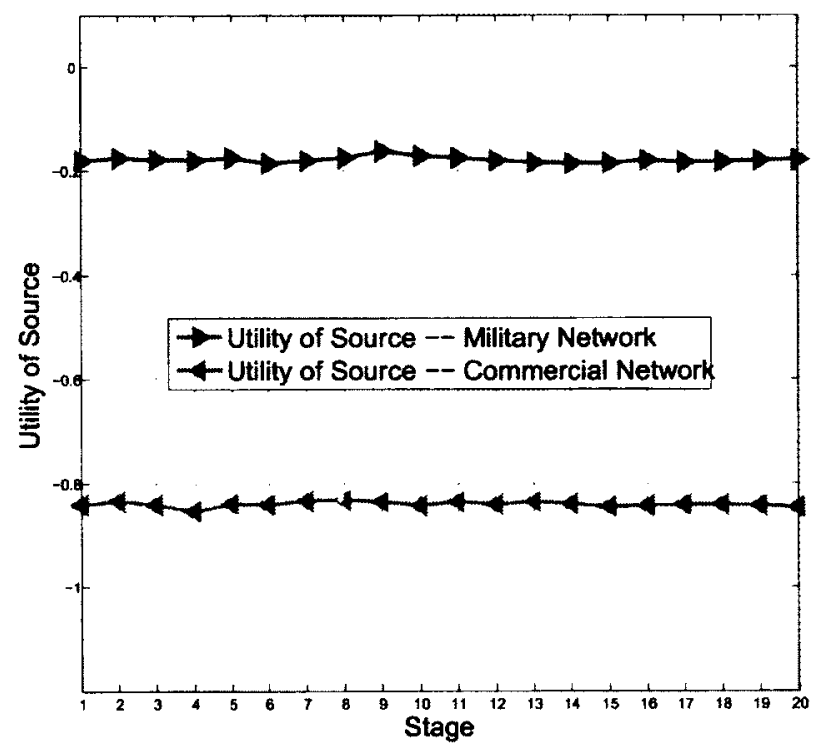

Figure 22: Comparison of dynamic total utility of the source in military and commercial networks

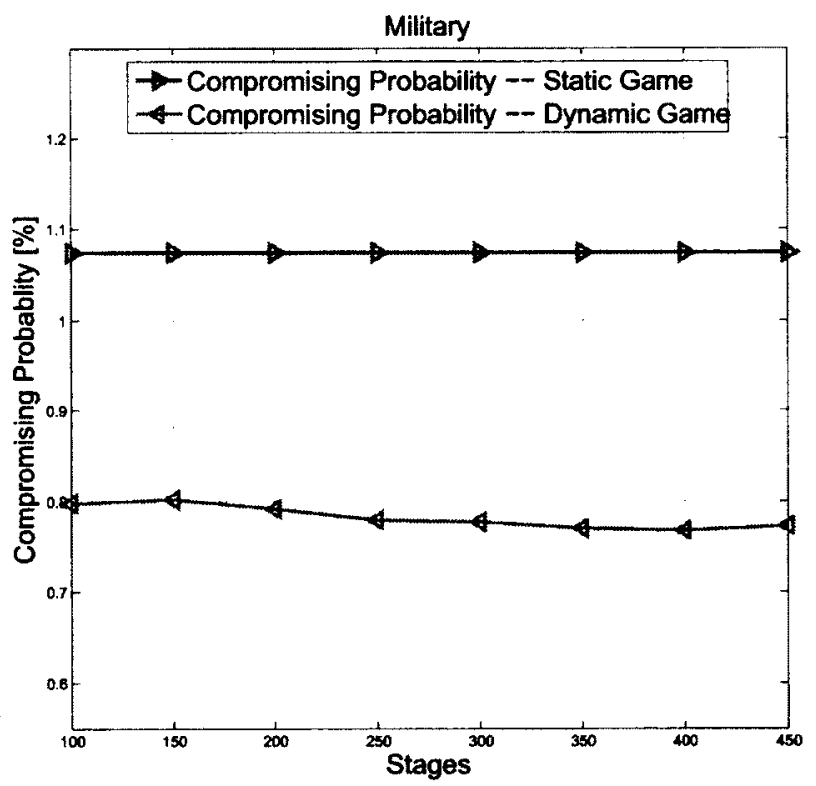

Figure 23: Impact of dynamic belief update in the maliciousness of relays on compromising probability of military network 


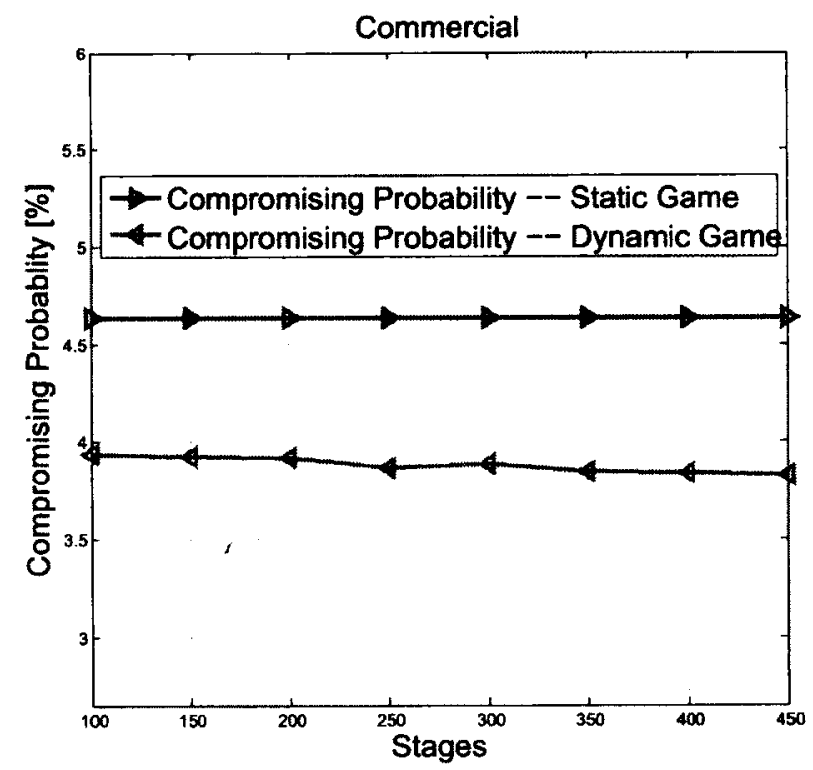

Figure 24: Impact of dynamic belief update in the maliciousness of relays on compromising probability of commercial network

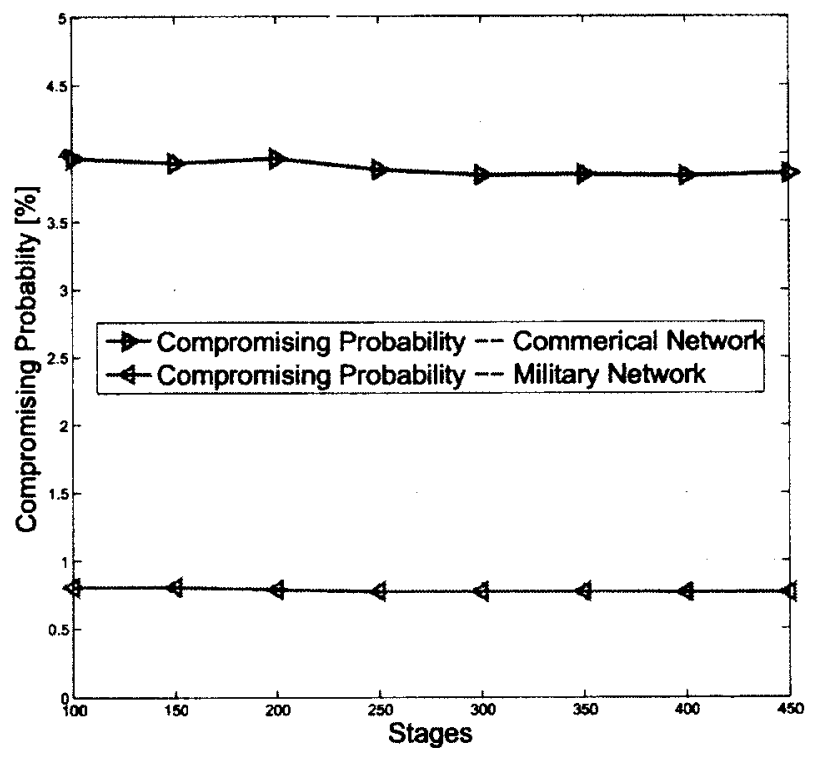

Figure 25: Dynamic compromising probability comparison between military and commercial network 


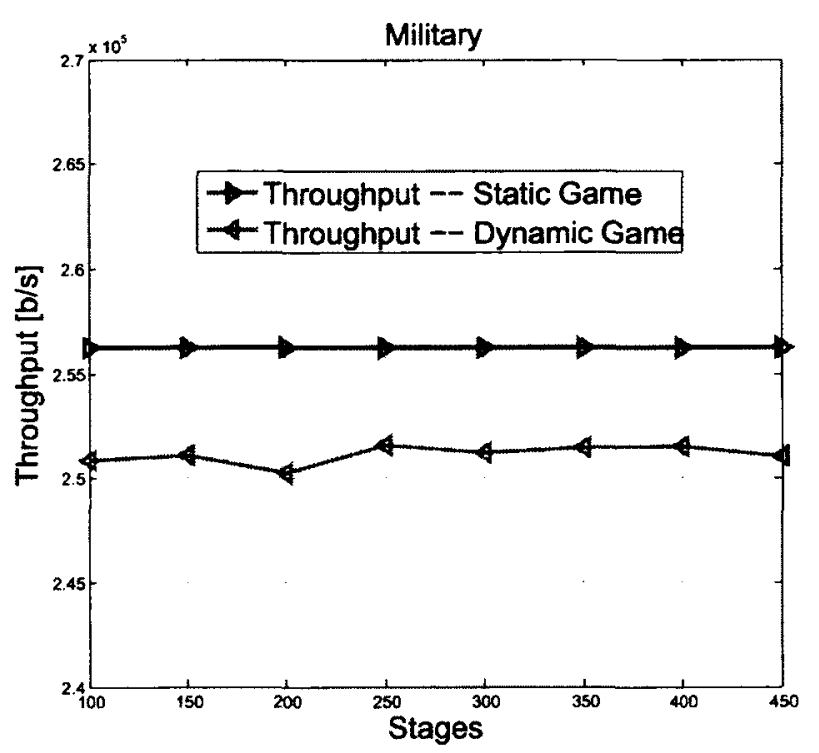

Figure 26: Impact of dynamic belief update in the maliciousness of relays on throughput of military network, $\mathrm{SNR}=30 \mathrm{~dB}$

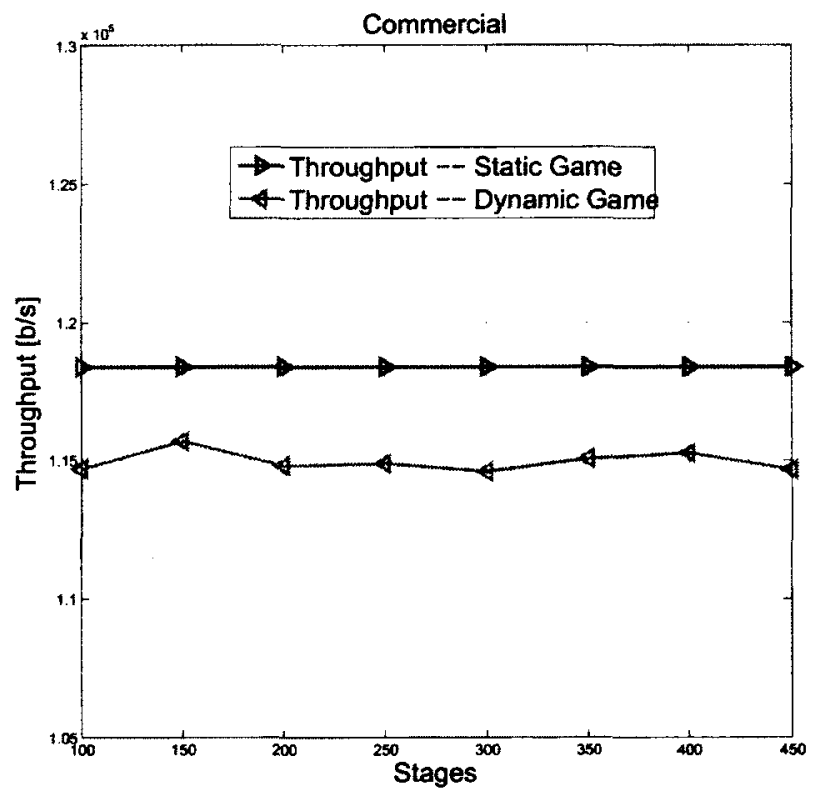

Figure 27: Impact of dynamic belief update in the maliciousness of relays on throughput of commercial network, SNR $=30 \mathrm{~dB}$ 


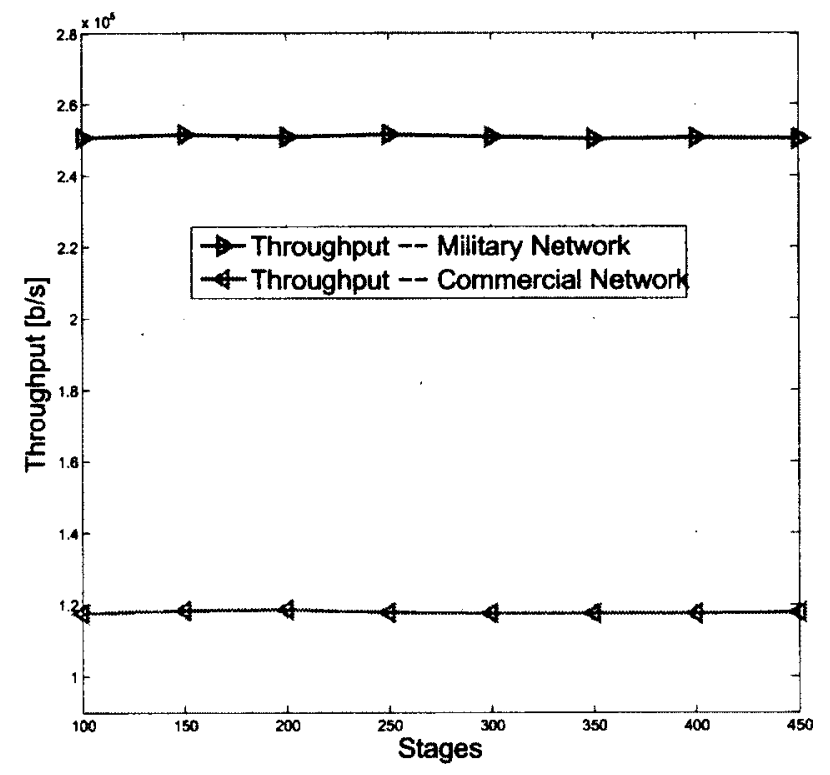

Figure 28: Dynamic throughput comparison between military and commercial network, $\mathrm{SNR}=30 \mathrm{~dB}$ 


\section{Chapter 5}

\section{Conclusions and Future Work}

\subsection{Conclusions}

In this thesis, we propose a quantitative approach based on game theory for security and QoS co-design in cooperative wireless communication networks, which considers the trade-off between system throughput and system security requirements. The proposed game theoretic approach overcomes the weaknesses of two extremes of the existing approaches that either focus on improving the system throughput without considering the security issues that arise in cooperative wireless communication networks or focus on designing stringent encryption and authentication protocols to ensure secure communication at the cost of the tremendous consumption of system computational resource. What is more, the proposed game theoretic approach provides a quantitative analysis on the attacking probability of the attacker on selected relay which is obtained by finding the Nash equilibrium of the proposed game theoretic approach.

To cope with the scenarios in which all relays are malicious, we propose a static game theoretic approach for security and QoS co-design. We formulate the attacking target selection and relay selection interaction between the attacker and the source 
as a noncooperative game, in which both participants try to maximize their total utility. We also perform an analysis on system throughput improvement. Based on the proposed static game theoretic approach, system throughput is improved while satisfying system security requirements. We also execute extensive simulations on two typical networks, military network and commercial network, to show the effectiveness of the proposed approach for security and QoS co-design. To cope with the scenarios in which there are cooperative relays and interactions between the source and the attacker, we propose a dynamic game theoretic approach for security and QoS co-design in which the source updates its beliefs in the maliciousness of relays based on its previous beliefs and its record of attacker's attacking histories on relays. By applying dynamic belief update, the source obtains more total utility in both military and commercial networks; and the compromising probability obtained by applying dynamic game theoretic approach is smaller than that obtained by applying static game theoretic approach since the source is prone to choose the less frequently attacked relays based on its belief updating results, though the system throughput obtained by applying the proposed dynamic game theoretic approach is slightly inferior to that obtained by applying the proposed static game theoretic approach.

\subsection{Future Work}

In future research, to cope with the attack initiated by multiple attackers in cooperative wireless communication networks, we would like to design more robust game theoretic approach for security and QoS co-design. The more robust game theoretic approach should be applicable to handle the networks with known and unknown number of attackers. New beliefs updating rules in the maliciousness of relays should be proposed based on the attack initiated by known and unknown number of multiple attackers. We would also like to extend the proposed game theoretic approach in 
this thesis that applies to two-hop cooperative wireless communication networks to multi-hop cooperative wireless communication networks and multi-hop mobile Ad hoc networks which may also suffer the attack initiated by single or multiple attackers on mobile nodes. We would like to extend the proposed game theoretic approach for routing nodes selection in mobile Ad hoc networks due to the existence of noncooperative nodes that would drop off arrival packets sent from the source or malicious nodes that would modify the arrival packets. Last but not the least, we would like to devise more accurate and pragmatic utility functions to depict the interactions between the source who acts the role of the defender through strategic relay selection based on its estimation on attackers' attacking strategies and multiple attackers. 


\section{List of References}

[1] R. Ramamoorthy, F. R. Yu, H. Tang, and P. C. Mason, "Combined authentication and quality of service in cooperative communication networks," in $E U C$, pp. $566-571,2010$.

[2] A. Nosratinia, T. E. Hunter, and A. Hedayat, "Cooperative communication in wireless networks," IEEE Communications Magazine, vol. 42, no. 10, pp. 74-80, 2004.

[3] J. N. Laneman, D. N. C. Tse, and G. W. Wornell, "Cooperative diversity in wireless networks: efficient protocols and outage behavior," IEEE Trans. Inform. Theory, vol. 50, pp. 3062-3080, 2004.

[4] T. E. Hunter, A. Nosratinia, and S. Member, "Diversity through coded cooperation," IEEE Trans. Wireless Commun, vol. 5, pp. 283-289, 2006.

[5] A. Bletsas, H. Shin, and M. Z. Win, "Cooperative Communications with OutageOptimal Opportunistic Relaying," IEEE Trans. Wireless Commun, vol. 6, no. 9, pp. 3450-3460, 2007.

[6] A. Bletsas, H. Shin, and M. Z. Win, "Cooperative diversity with opportunistic relaying," in Proc. IEEE WCNC06, pp. 1034-1039, 2006.

[7] K. Woradit, T. Quek, W. Suwansantisuk, M. Win, L. Wuttisittikulkij, and H. Wymeersch, "Outage behavior of selective relaying schemes," IEEE Trans. Wireless Commun, vol. 8, pp. 3890 -3895, august 2009.

[8] Y. Wang, G. Attebury, and B. Ramamurthy, "A survey of security issues in wireless sensor networks," IEEE Communications Surveys \& Tutorials, vol. 8, pp. 2-23, 2006.

[9] W. Stallings, Cryptography and network security. Prentice Hall, second ed., 1999. 
[10] G. Schäfer, Security in Fixed and Wireless Networks: An Introduction to securing data communications. John Wiley \& Sons, 2004.

[11] N. Aboudagga, M. T. Refaei, M. Eltoweissy, L. A. Dasilva, and J. jacques Quisquater, "Authentication protocols for ad hoc networks: taxonomy and research issues," in Q2SWinet 05: Proceedings of the 1st ACM international workshop on Quality of Service and security in wireless and mobile networks, pp. 96104, ACM Press, 2005.

[12] R. Rivest, A. Shamir, and L. Adleman, "A method for obtaining digital signatures and public-key cryptosystems," Communications of the $A C M$, vol. 21, pp. $120-126,1978$.

[13] M. O. Rabin, Digitalized signatures and public-key functions as intractable as factorization. MIT Laboratory for Computer Science, 1979.

[14] N. Koblitz, "Elliptic Curve Cryptosystems," Mathematics of Computation, vol. 48 , no. 177 , pp. 203-209, 1987.

[15] A. Shamir, "Identity-Based Cryptosystems and Signature Schemes," Advances in Cryptology, pp. 47-53, 1985.

[16] D. Boneh and M. K. Franklin, "Identity-Based Encryption from the Weil Pairing," in Proceedings of the 21st Annual International Cryptology Conference on Advances in Cryptology, CRYPTO '01, (London, UK), pp. 213-229, SpringerVerlag, 2001.

[17] C. Cocks, "An identity based encryption scheme based on quadratic residues," in IMA INT. CONF, pp. 360-363, Springer-Verlag, 2001.

[18] A. Perrig, R. Canetti, J. D. Tygar, and D. Song, "The TESLA broadcast authentication protocol," RSA CryptoBytes, vol. 5, no. Summer, 2002.

[19] S. Zhu, S. Xu, S. Setia, and S. Jajodia, "LHAP: A lightweight hop-by-hop authentication protocol for ad-hoc networks.," in ICDCS Workshops'03, pp. 749-749, 2003.

[20] R. Akbani, T. Korkmaz, and G. V. S. Raju, "HEAP: A packet authentication scheme for mobile ad hoc networks," Ad Hoc Netw., vol. 6, pp. 1134-1150, 2008.

[21] T. Heer, S. Götz, O. G. Morchon, and K. Wehrle, "ALPHA: An adaptive and lightweight protocol for hop-by-hop authentication," in Proceedings of the 2008 ACM CoNEXT Conference, CoNEXT '08, pp. 23:1-23:12, ACM, 2008. 
[22] D. R. Stinson, Cryptography: Theory and Practice, Second Edition. Chapman \& Hall/CRC, Feb. 2002.

[23] L. Lamport, "Password authentication with insecure communication," $A C M$ Commun., vol. 24, pp. 770-772, Nov. 1981.

[24] D. E. Eastlake and P. E. Jones, "US Secure Hash Algorithm 1 (SHA1)."

[25] R. C. Merkle, "A digital signature based on a conventional encryption function," in Advances in Cryptology - Crypto '87, pp. 369-378, Springer-Verlag, 1987.

[26] R. C. Merkle, "A certified digital signature," in Proceedings in Advances in Cryptology, CRYPTO '89, pp. 218-238, 1989.

[27] O. Morgenstern and J. Von Neumann, Theory of Games and Economic Behavior. Princeton University Press, 3 ed., May 1944.

[28] J. Nash, "Two person cooperative games," Econometrica, vol. 21, pp. 128-140, January 1953.

[29] M. Felegyhazi and J.-P. Hubaux, "Game theory in wireless networks: A Tutorial," Tech. Rep. EPFL, 2006.

[30] N. Nisan, T. Roughgarden, E. Tardos, and V. V. Vazirani, Algorithmic Game Theory. Cambridge University Press, 2007.

[31] R. Gibbons, Game Theory for Applied Economists. Princeton University Press, 1992.

[32] T. Basar and G. J. Olsder, Dynamic Noncooperative Game Theory (Classics in Applied Mathematics). Soc for Industrial \& Applied Math, 2 ed., Jan. 1999.

[33] M. J. Osborne and A. Rubinstein, A Course in Game Theory. The MIT Press, July 1994.

[34] R. Gibbons, A Primer in Game Theory. FT Prentice Hall, 1992.

[35] J. C. Harsanyi, "Games with Incomplete Information Played by "Bayesian" Players, I-III. Part I. The Basic Model," Management Science, vol. 14, pp. 159-182, Nov. 1967.

[36] L. Chen and J. Leneutre, "A game theoretical framework on intrusion detection in heterogeneous networks," IEEE Trans. Info. For. Sec., vol. 4, pp. 165-178, June 2009. 
[37] Y. Liu, C. Comaniciu, and H. Man, "A bayesian game approach for intrusion detection in wireless ad hoc networks," in Proceeding from the 2006 workshop on Game theory for communications and networks, GameNets '06, (New York, NY, USA), ACM, 2006.

[38] C. Zouridaki, B. L. Mark, and M. Hejmo, "A quantitative trust establishment framework for reliable data packet delivery in manets," in Proceedings of the Third ACM Workshop on Security of Ad Hoc and Sensor Networks (SASN, pp. 110, ACM, 2005.

[39] S. Buchegger and J.-Y. L. Boudec, "A robust reputation system for mobile adhoc networks," tech. rep., Proceedings of P2PEcon, 2003.

[40] E. W. Dijkstra, "Recursive programming," Numerische Mathematik, vol. 2, pp. 312-318, 1960.

[41] J. F. Nash, "Equilibrium points in n-person games," in Proceedings of the $\mathrm{Na}$ tional Academy of Sciences of the United States of America, 1950.

[42] J. B. Rosen, "Existence and uniqueness of equilibrium points for concave nperson games," Econometrica, vol. 33, no. 3, pp. 520-534, 1965.

[43] P. Paruchuri, "Playing games for security: An efficient exact algorithm for solving bayesian stackelberg games," in AAMAS, 2008.

[44] M. Z. Win, S. Member, and J. H. Winters, "Virtual branch analysis of symbol error probability for hybrid selection/maximal-ratio combining in rayleigh fading," IEEE Trans. Commun, vol. 49, pp. 1926-1934, 2001.

[45] D. Tse and P. Viswanath, "Fundamentals of Wireless Communications," 2004.

[46] P. Herhold, E. Zimmermann, and G. Fettweis, "A simple cooperative extension to wireless relaying," in International Zurich Seminar on Communications (IZS), pp. 36-39, 2004.

[47] J. G. Kim and M. Krunz, "Delay Analysis of Selective Repeat ARQ for a Markovian Source Over a Wireless Channel," IEEE Trans. Veh. Technol, vol. 49, pp. 1968-1981, 1999.

[48] J. F. Kurose and K. W. Ross, Computer Networking: A Top-Down Approach (4th Edition). Addison Wesley, L ed., Apr. 2007. 
[49] H. Liu, H. Ma, M. E. Zarki, and S. Gupta, "Error control schemes for networks: an overview," Mob. Netw. Appl., vol. 2, pp. 167-182, Oct. 1997.

[50] S. Lin, D. Costello, and M. Miller, "Automatic-repeat-request error-control schemes," IEEE Communications Magazine, vol. 22, no. 12, pp. 5-17, 1984. 


\section{Appendix A}

\section{Simulation Programs}

BeliefUpdate.m

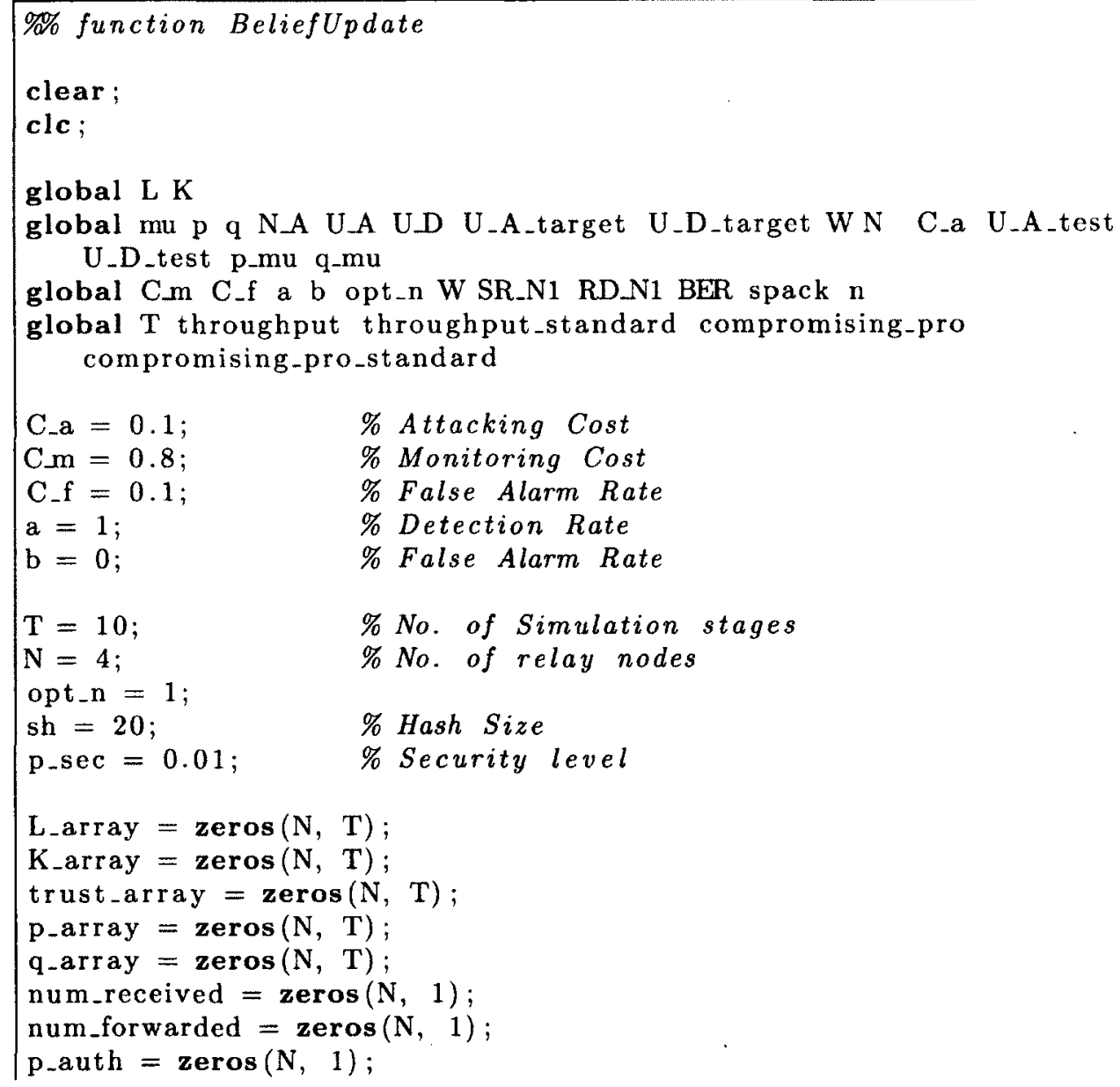




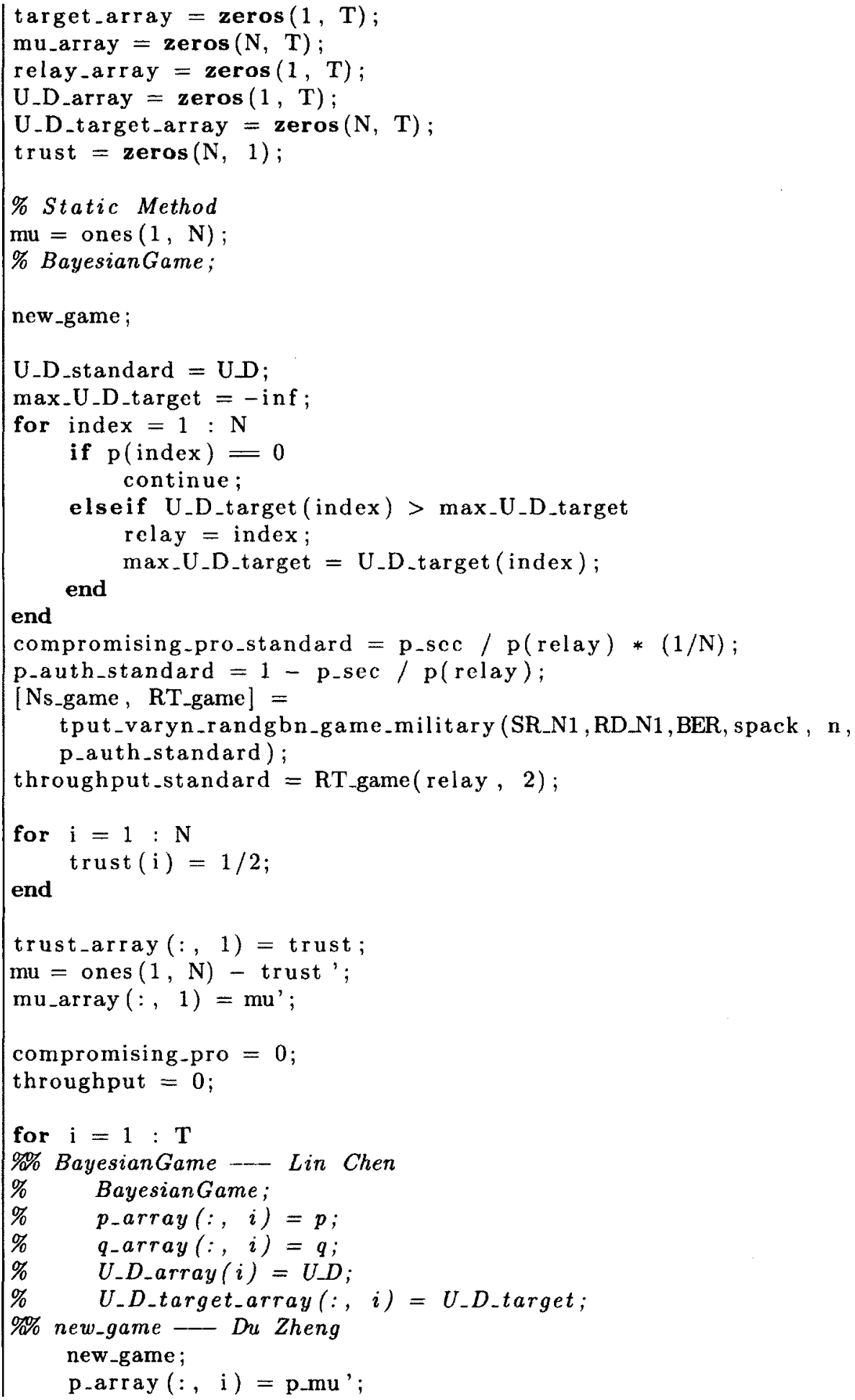




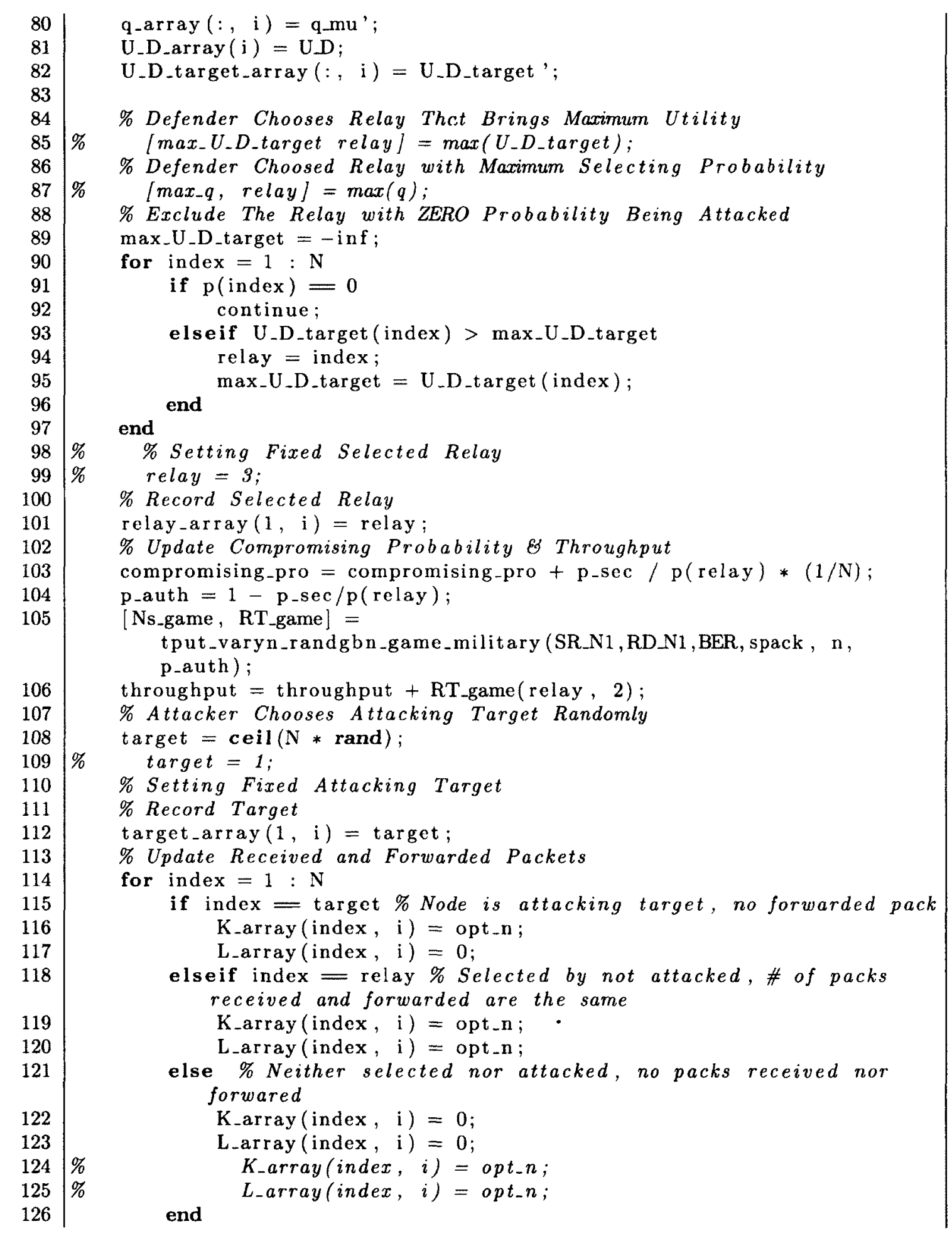




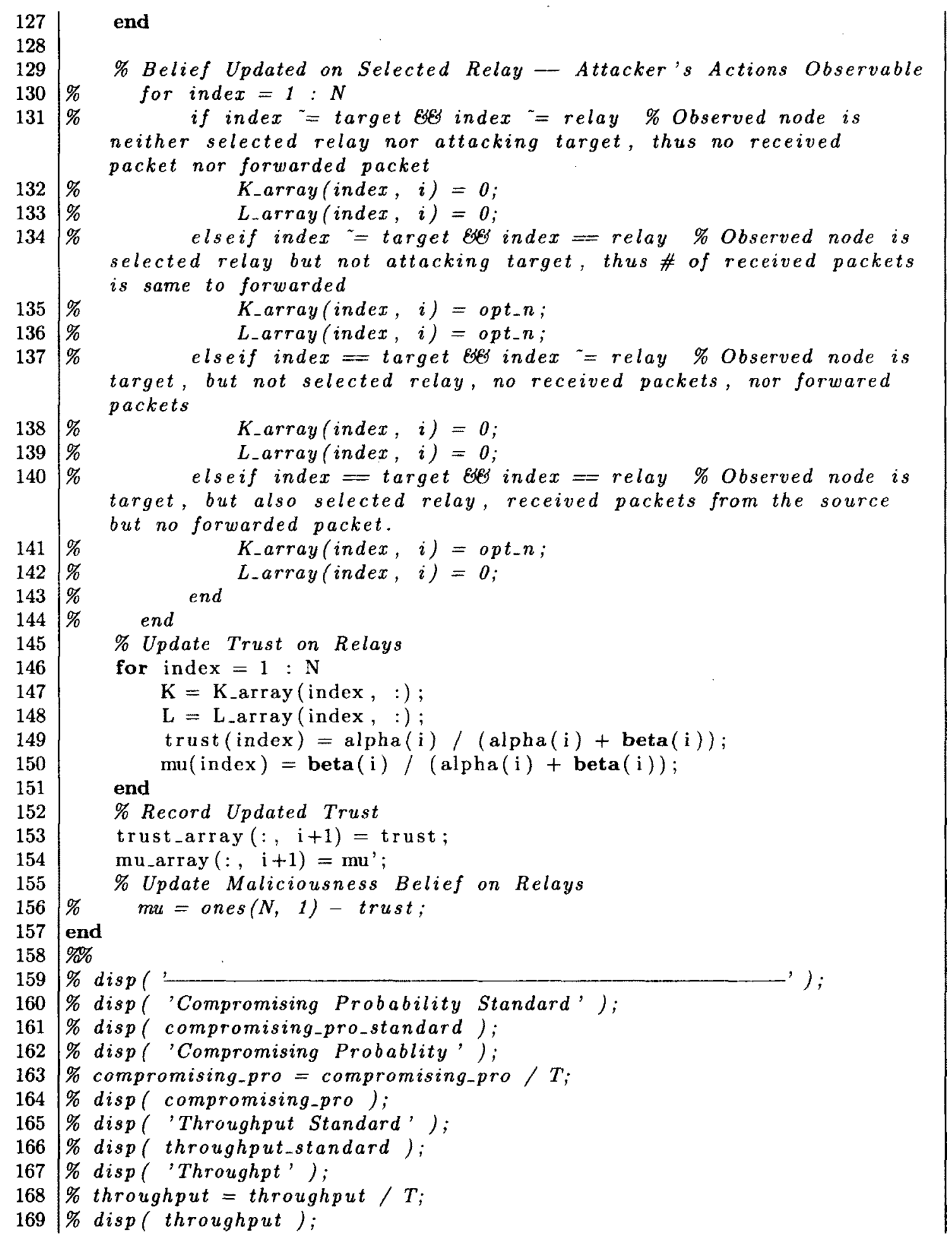




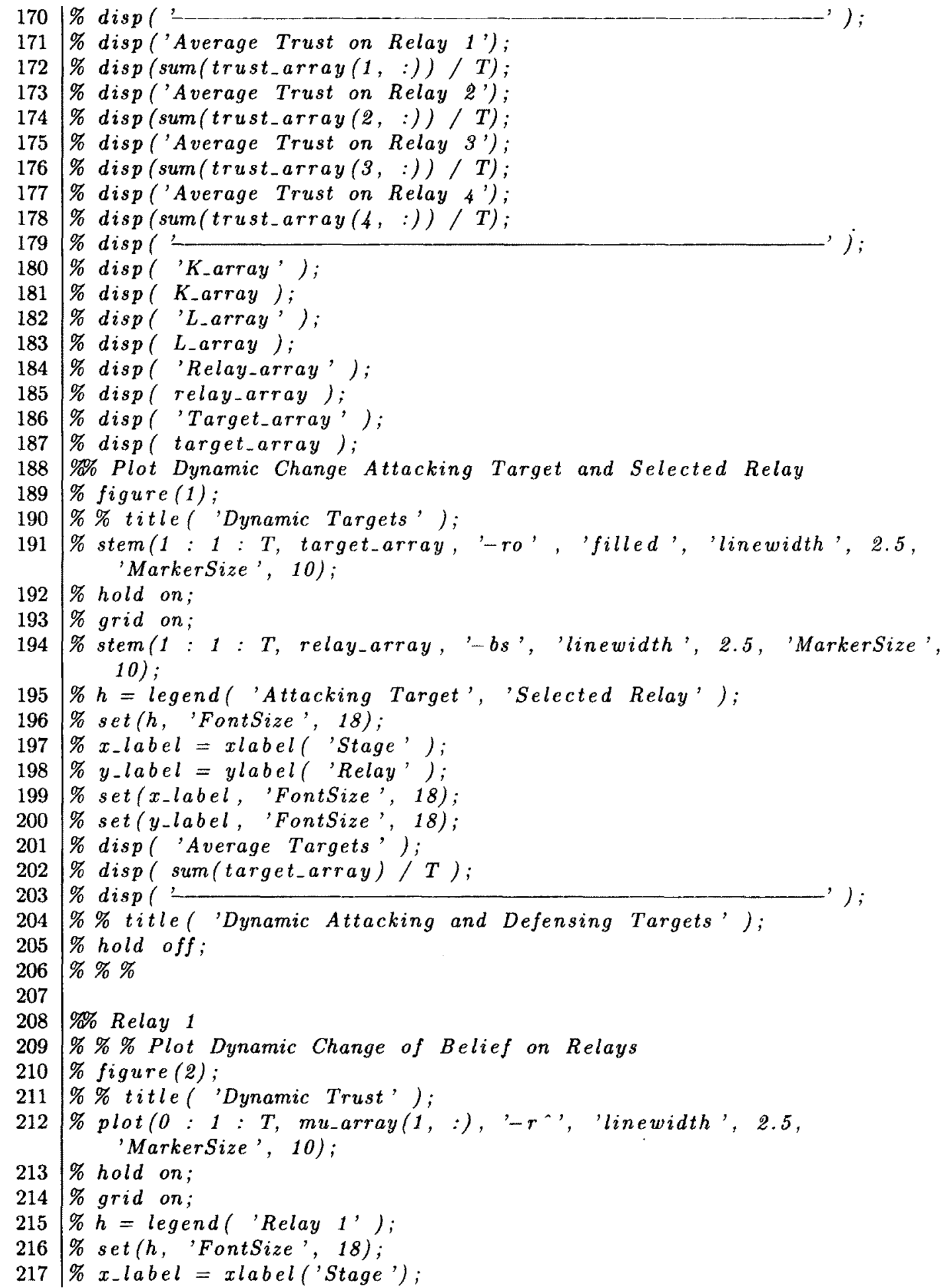




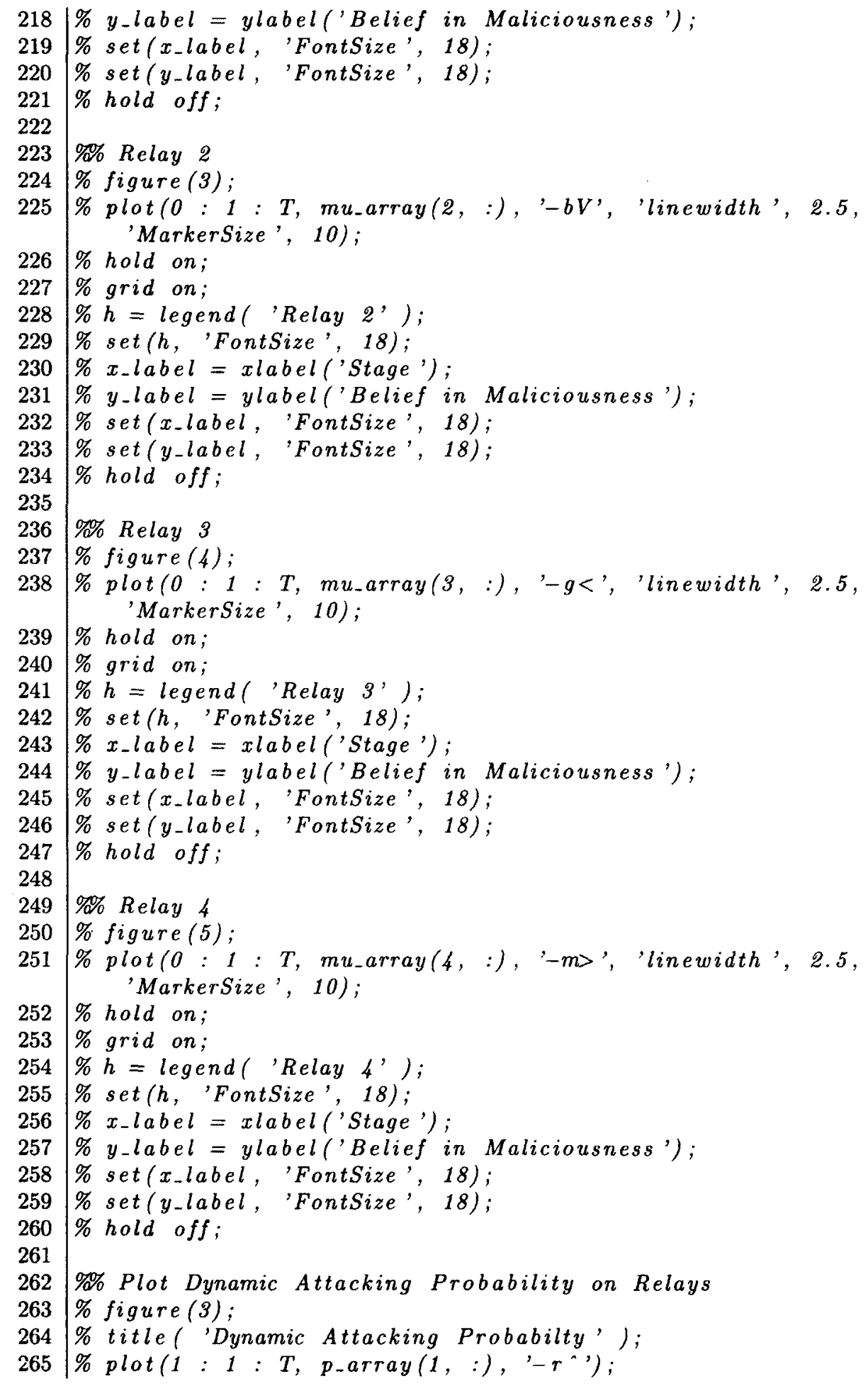




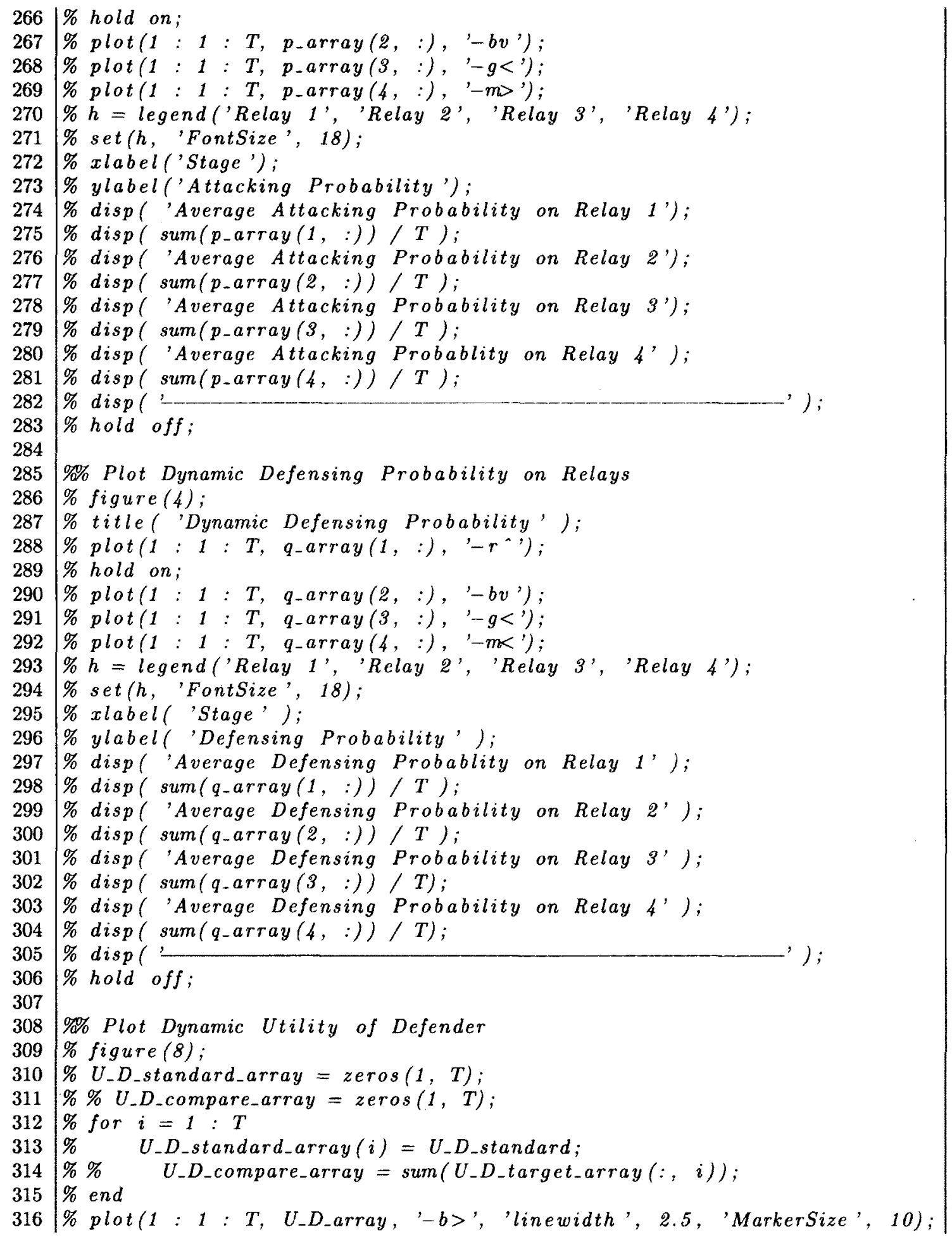




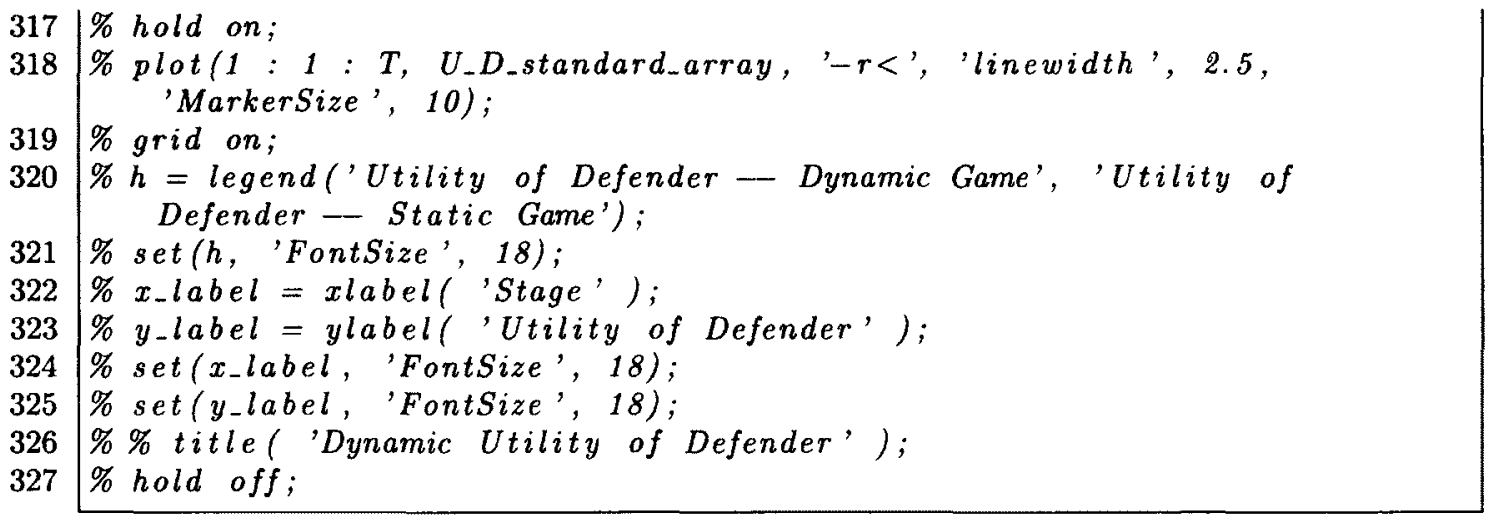

BayesianGame.m

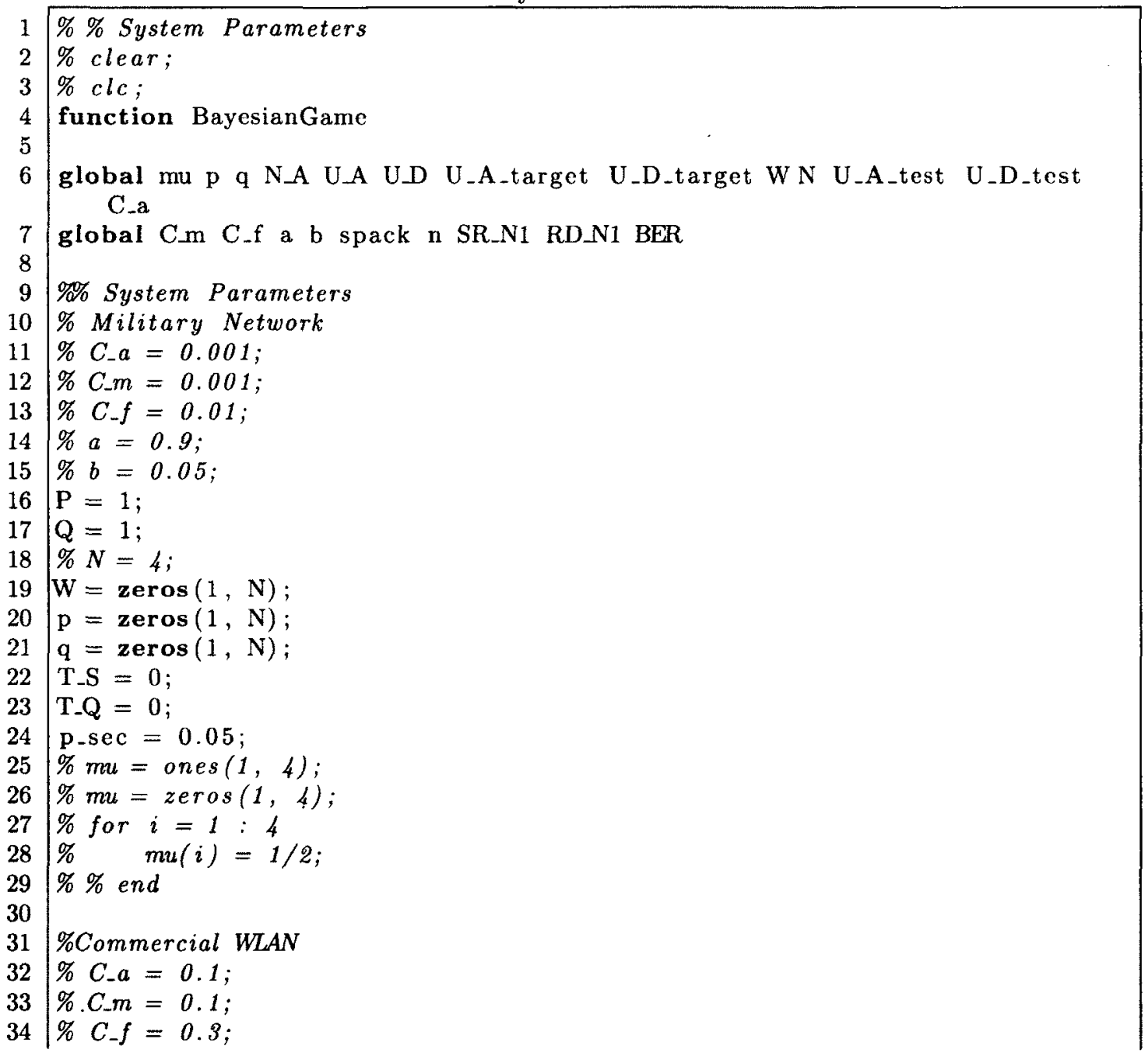




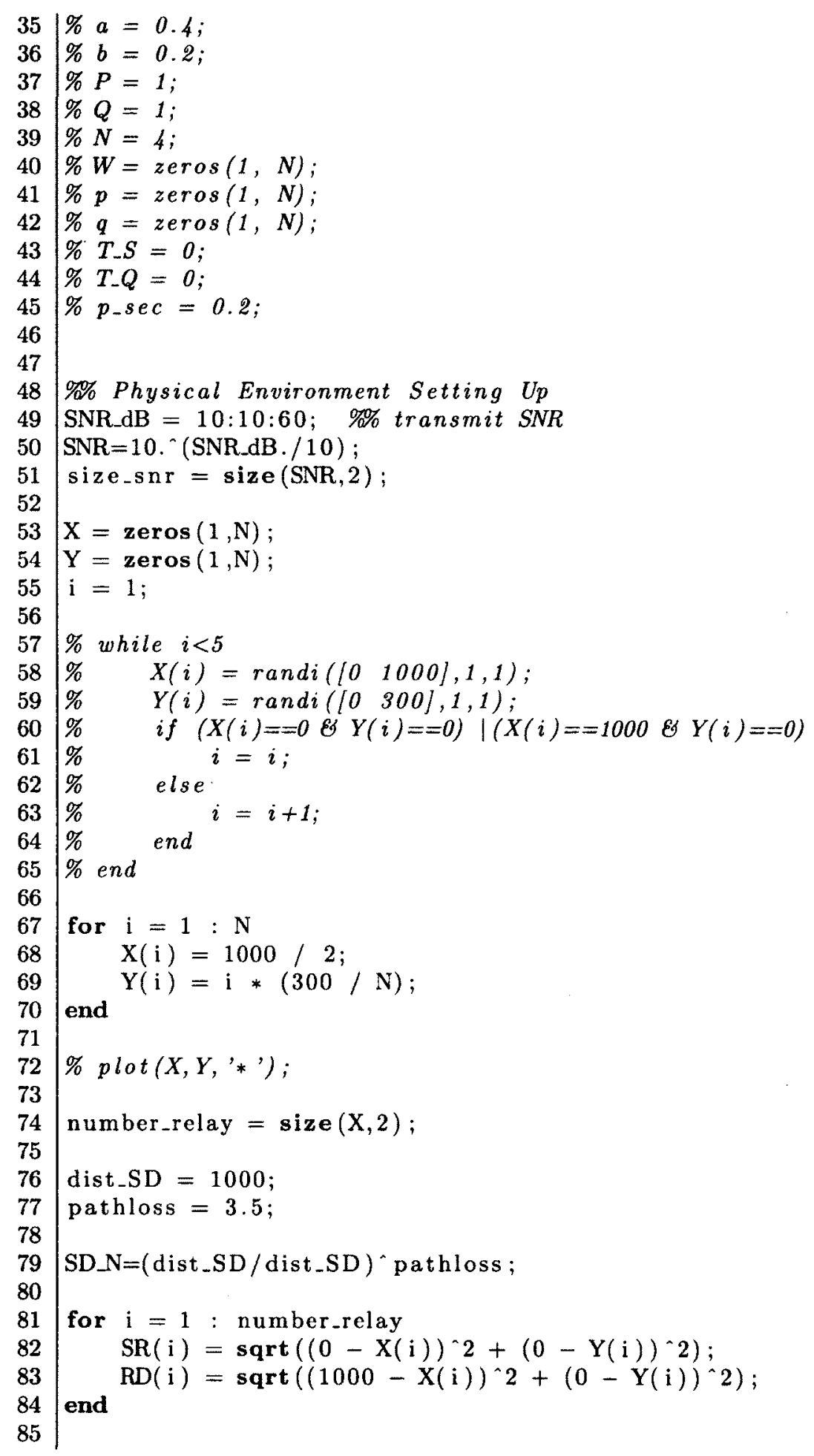




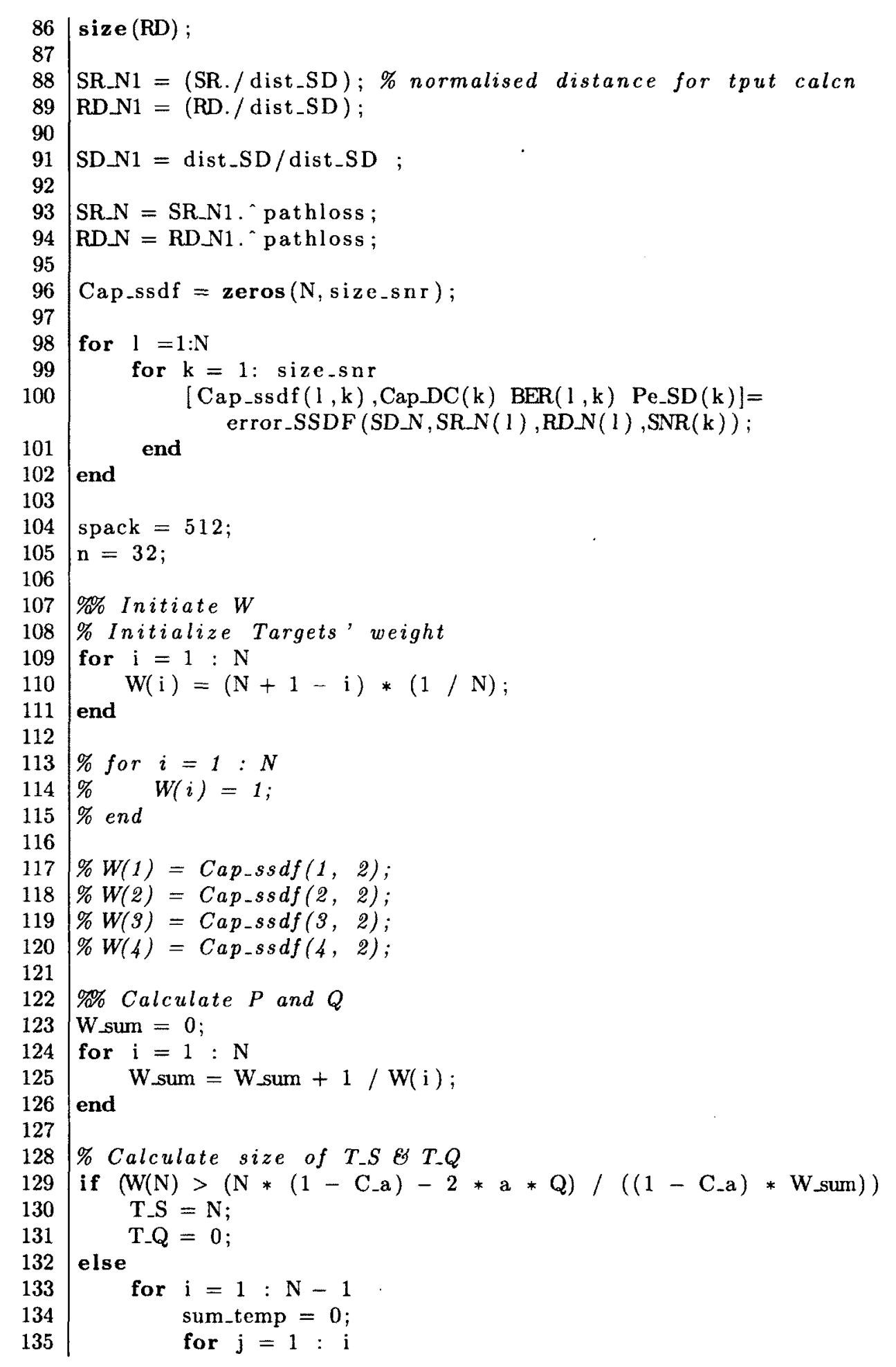




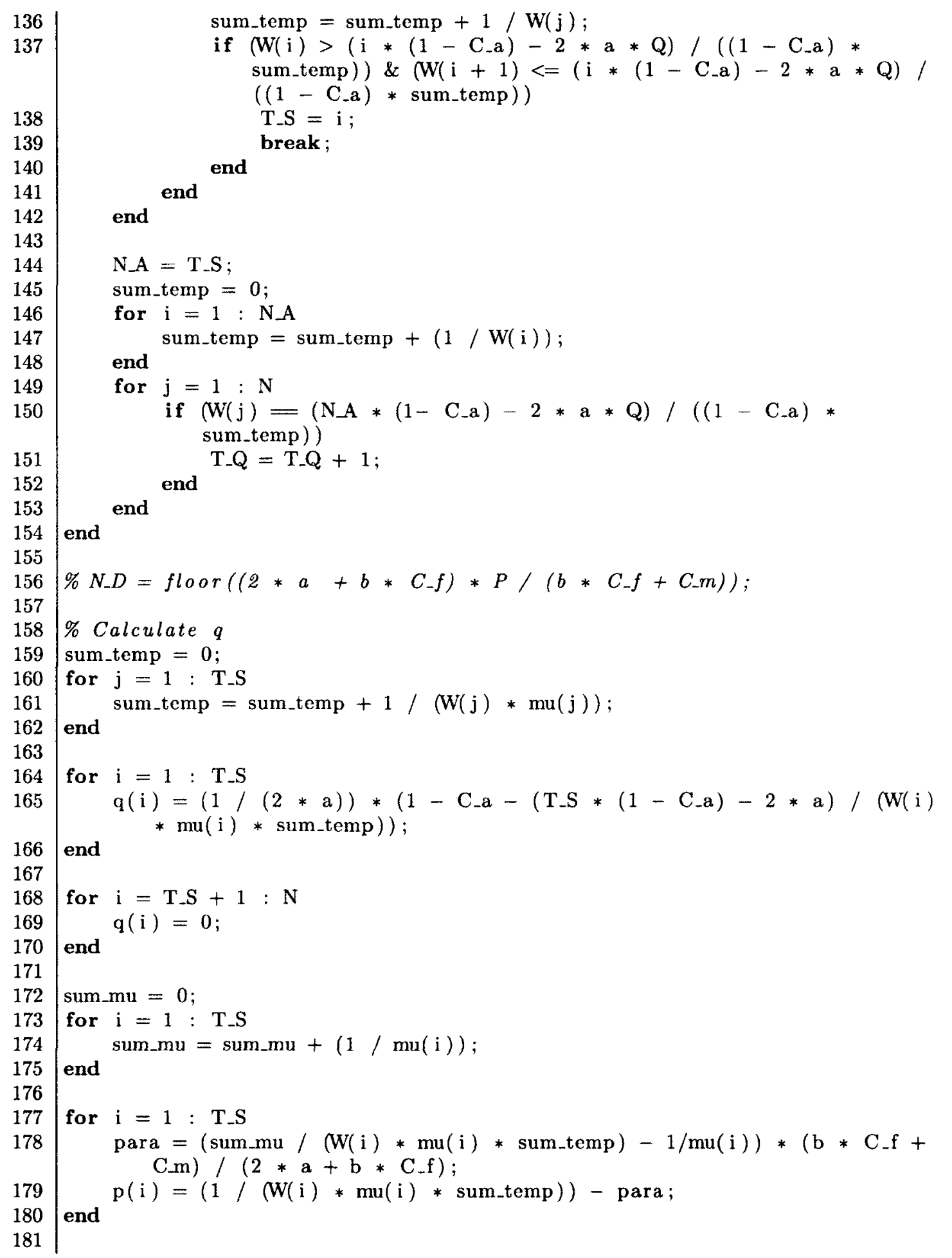




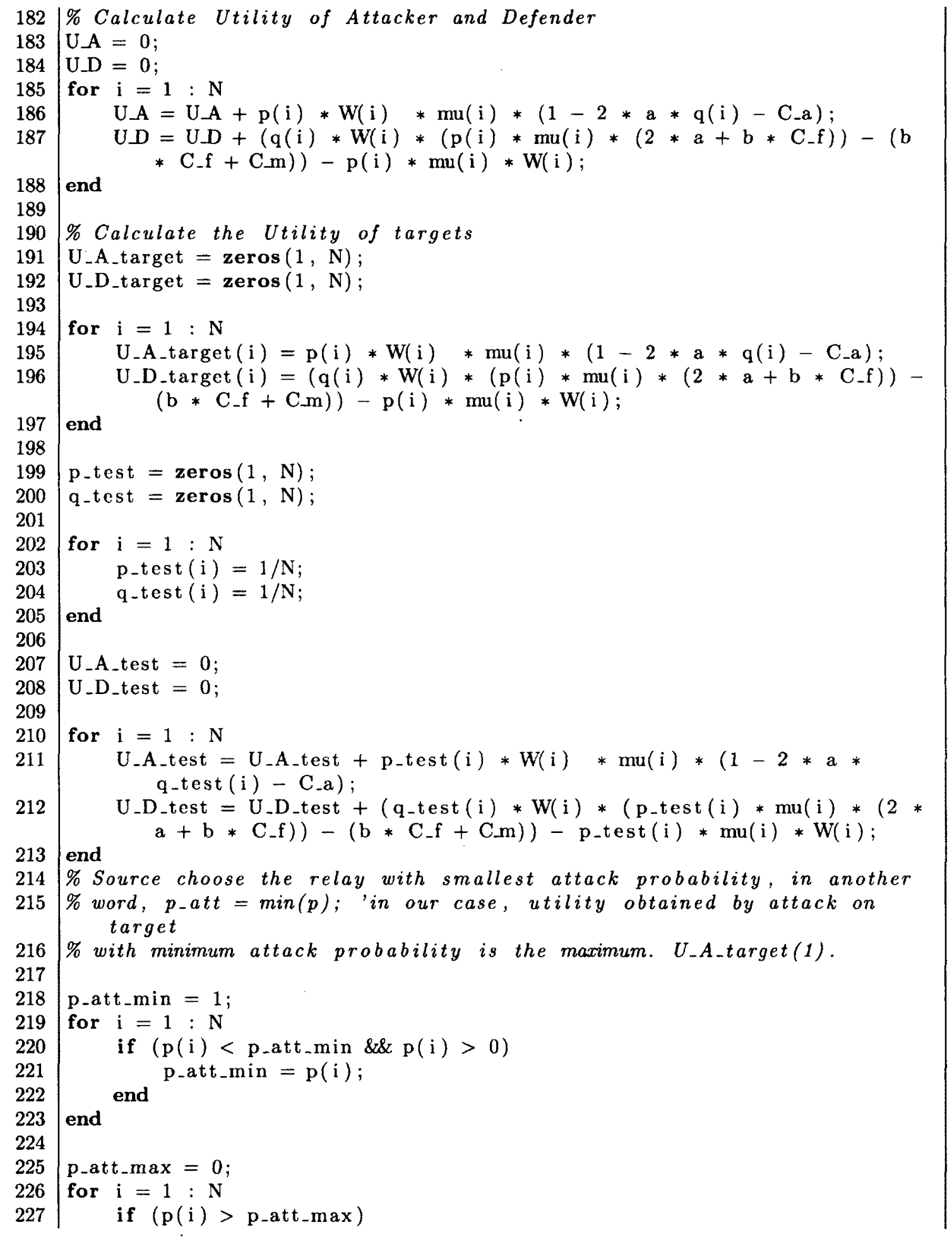


$228 \quad$ p_att $\max =\mathrm{p}(\mathrm{i})$;

229 end

230 end 